\title{
Database Investigation of Halogen Bonding and Halogen $\cdots$ Halogen Interactions between Porphyrins: Emergence of Robust Supramolecular Motifs and Frameworks
}

\author{
Toni S. Spilfogel, Hatem M. Titi and Tomislav Friščić
}

\section{Table of Contents}

1. Figures and tables for chlorine-based interactions .......................................................... 2

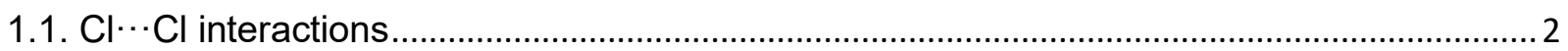

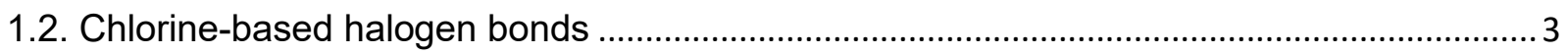

2. Figures and tables for bromine-based interactions ...................................................... 4

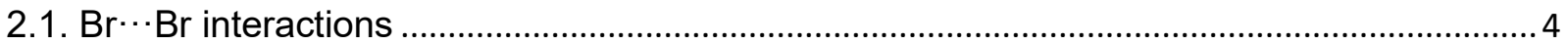

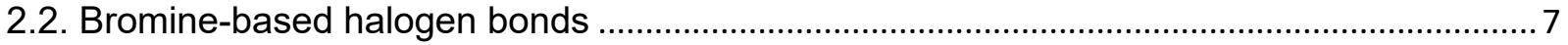

3. Figures and tables for iodine-based interactions .......................................................... 8

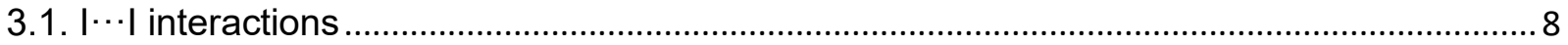

3.2. lodine-based halogen bonding interactions.................................................................... 11

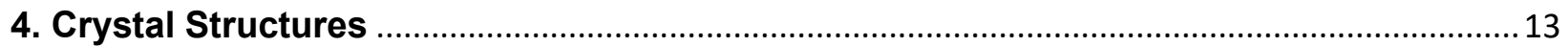

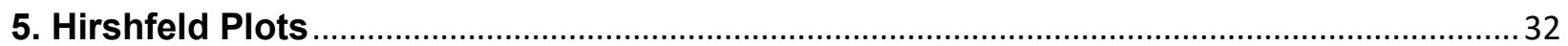

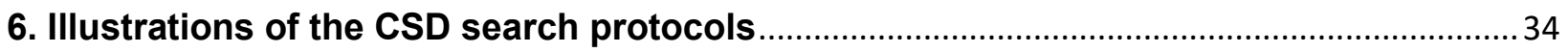




\section{Figures and tables for chlorine-based interactions}

\section{1. $\mathrm{Cl} \cdots \mathrm{Cl}$ interactions}

a)

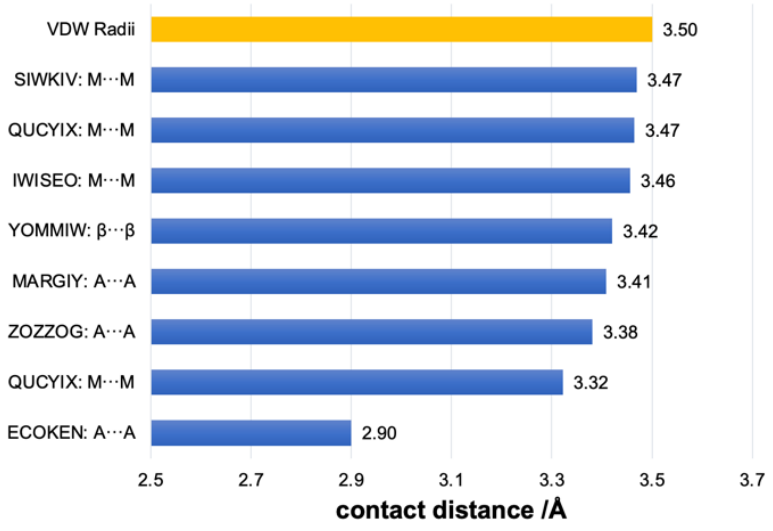

b)

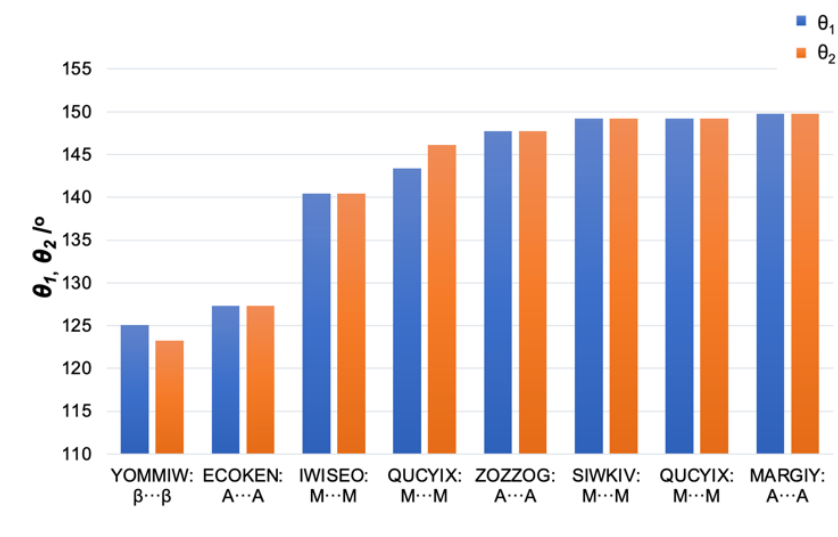

Figure S1. Histograms of geometrical parameters for Type I Cl $\cdots \mathrm{Cl}$ interactions: a) interatomic distances and b) $\theta$ angles.

a)

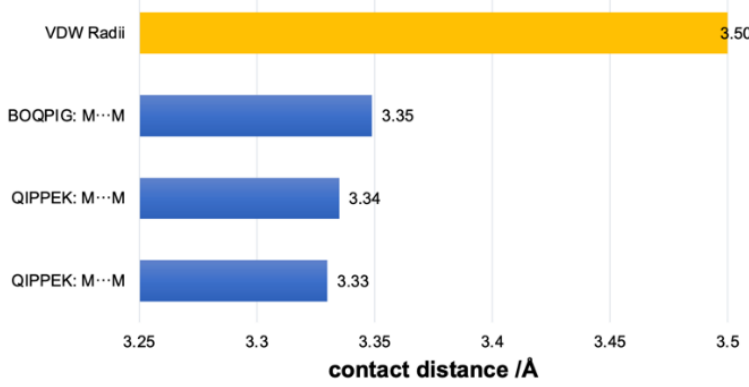

b)

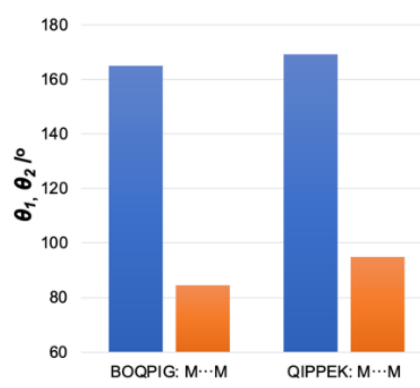

$=\theta_{1}$
$=\theta_{2}$

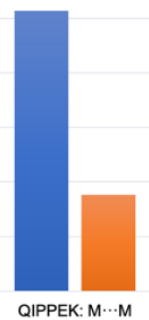

Figure S2. Histograms of geometrical parameters for Type II Cl $\cdots \mathrm{Cl}$ interactions: a) interatomic distances and b) $\theta$ angles.

Table S1. Overview of CSD database entries that contain Type I Cl $\cdots \mathrm{Cl}$ interactions.

\begin{tabular}{l|cccccccc}
\multicolumn{1}{c|}{ Entry } & Geometry & $\begin{array}{c}\text { Space } \\
\text { Group }\end{array}$ & $\mathbf{C}-\mathbf{C l}(\mathbf{A})$ & $\mathbf{C}-\mathbf{C l}(\mathbf{A})$ & $\boldsymbol{\theta}_{\mathbf{1}}\left(\mathbf{(}^{\circ}\right)$ & $\boldsymbol{\theta}_{\mathbf{2}}\left({ }^{\circ}\right)$ & $\begin{array}{c}\mathbf{C l} \cdots \mathbf{C l} \\
(\mathbf{A})\end{array}$ & $\boldsymbol{\varphi}\left(^{(}\right)$ \\
\hline ECOKEN & $\mathrm{A} \cdots \mathrm{A}$ & $P-1$ & 1.75 & 1.75 & 127.3 & 127.3 & 2.90 & 180.0 \\
IWISEO & $\mathrm{M} \cdots \mathrm{M}$ & $P-1$ & 1.74 & 1.74 & 140.5 & 140.5 & 3.46 & -180.0 \\
MARGIY & $\mathrm{A} \cdots \mathrm{A}$ & $P 21 / \mathrm{n}$ & 1.75 & 1.75 & 149.8 & 149.8 & 3.41 & -180.0 \\
QUCYIX & $\mathrm{M} \cdots \mathrm{M}$ & $P 21 / \mathrm{c}$ & 1.75 & 1.74 & 143.4 & 146.1 & 3.47 & 176.6 \\
QUCYIX & $\mathrm{M} \cdots \mathrm{M}$ & $P 21 / \mathrm{C}$ & 1.74 & 1.74 & 149.2 & 149.2 & 3.32 & 180.0 \\
SIWKIV & $\mathrm{M} \cdots \mathrm{M}$ & $I 222$ & 1.73 & 1.73 & 149.2 & 149.2 & 3.47 & 134.5 \\
YOMMIW & $\beta \cdots \beta$ & $P 21 / \mathrm{c}$ & 1.71 & 1.72 & 125.1 & 123.2 & 3.42 & 132.6 \\
ZOZZOG & $\mathrm{A} \cdots \mathrm{A}$ & $P-1$ & 1.74 & 1.74 & 147.7 & 147.7 & 3.38 & 180.0
\end{tabular}


Table S2. Overview of CSD database entries that contain Type I Cl $\cdots \mathrm{Cl}$ interactions.

\begin{tabular}{c|cccccccc} 
Entry & Geometry & $\begin{array}{c}\text { Space } \\
\text { Group }\end{array}$ & $\mathbf{C}-\mathbf{C l}(\mathbf{A})$ & $\mathbf{C}-\mathbf{C l}(\boldsymbol{A})$ & $\boldsymbol{\theta}_{\mathbf{1}}\left(^{(}\right)$ & $\boldsymbol{\theta}_{\mathbf{2}}\left(^{\circ}\right)$ & $\mathbf{C l} \cdots \mathbf{C l}(\boldsymbol{\AA})$ & $\boldsymbol{\varphi}\left(^{(}\right)$ \\
\hline BOQPIG & $\mathrm{M} \cdots \mathrm{M}$ & $P 22_{1} / \mathrm{n}$ & 1.78 & 1.78 & 165.0 & 84.4 & 3.35 & -17.8 \\
QIPPEK & $\mathrm{M} \cdots \mathrm{M}$ & $P 2_{1}$ & 1.73 & 1.75 & 162.8 & 95.3 & 3.34 & 55.2 \\
QIPPEK & $\mathrm{M} \cdots \mathrm{M}$ & $P 2_{1}$ & 1.75 & 1.75 & 169.1 & 94.9 & 3.33 & 0.8
\end{tabular}

Table S3. Overview of all CSD database entries that contain $\mathrm{Cl} \cdots \mathrm{Cl}$ contacts.

\begin{tabular}{|c|c|c|c|c|c|c|c|}
\hline Name & Space Group & C-CI $(A)$ & C-Cl $(\AA)$ & $\theta_{1}\left(^{\circ}\right)$ & $\theta_{2}\left(^{\circ}\right)$ & $\mathrm{Cl} \cdots \mathrm{Cl}(\mathrm{A})$ & $\varphi\left({ }^{\circ}\right)$ \\
\hline ABASOO & $P 2_{1} / a$ & 1.73 & 1.75 & 163.1 & 156.8 & 3.33 & 123.5 \\
\hline AGUZAD & $C 2 / c$ & 1.73 & 1.73 & 127.3 & 87.4 & 3.49 & 137.0 \\
\hline BOQPIG & $P 2_{1} / \mathrm{C}$ & 1.78 & 1.78 & 84.4 & 165.0 & 3.35 & -17.8 \\
\hline DAFBOC & $P-1$ & 1.73 & 1.72 & 128.2 & 145.9 & 3.42 & 124.1 \\
\hline ECOKEN & $P-1$ & 1.75 & 1.75 & 127.3 & 127.3 & 2.90 & 180.0 \\
\hline ECUBAH & $14{ }_{1} / \mathrm{a}$ & 1.71 & 1.71 & 158.7 & 110.3 & 3.17 & 13.5 \\
\hline ECUBAH & $14_{1} / \mathrm{a}$ & 1.73 & 1.73 & 84.9 & 84.9 & 3.39 & -180.0 \\
\hline GAMBOL & $P-1$ & 1.73 & 1.73 & 85.2 & 85.2 & 3.39 & -180.0 \\
\hline IWISEO & $P-1$ & 1.74 & 1.74 & 140.5 & 140.5 & 3.46 & -180.0 \\
\hline LIRGIE & $P-1$ & 1.71 & 1.70 & 89.4 & 79.8 & 3.39 & 58.7 \\
\hline LIRGIE & $P-1$ & 1.71 & 1.72 & 91.5 & 81.6 & 3.50 & 51.2 \\
\hline LUBNAX & $P-1$ & 1.73 & 1.76 & 72.5 & 154.9 & 3.49 & 104.4 \\
\hline MARGIY & $P 2_{1} / \mathrm{n}$ & 1.75 & 1.75 & 149.8 & 149.8 & 3.41 & -180.0 \\
\hline MAVQOR & $P-1$ & 1.75 & 1.75 & 159.1 & 159.3 & 3.48 & 127.2 \\
\hline POJDOH & $P 1$ & 1.74 & 1.75 & 155.0 & 156.1 & 3.26 & 177.3 \\
\hline PUNMIW & $P 2_{1} / \mathrm{n}$ & 1.70 & 1.71 & 121.0 & 159.4 & 3.33 & -135.0 \\
\hline PUNMIW & $P 2_{1} / \mathrm{n}$ & 1.73 & 1.71 & 171.5 & 112.5 & 3.40 & 140.4 \\
\hline QIPPEK & $P 21$ & 1.73 & 1.75 & 95.3 & 162.8 & 3.34 & 55.2 \\
\hline QIPPEK & $P 2_{1}$ & 1.75 & 1.75 & 94.9 & 169.1 & 3.33 & 0.8 \\
\hline QUCYIX & $P 2_{1} / \mathrm{C}$ & 1.75 & 1.74 & 143.4 & 146.1 & 3.47 & 176.6 \\
\hline QUCYIX & $P 2_{1} / \mathrm{C}$ & 1.74 & 1.74 & 149.2 & 149.2 & 3.32 & 180.0 \\
\hline QUCYOD & $P-1$ & 1.74 & 1.74 & 157.6 & 157.6 & 3.34 & 180.0 \\
\hline QUCYOD & $P-1$ & 1.75 & 1.75 & 145.6 & 153.7 & 3.28 & 178.7 \\
\hline SIWKIV & 1222 & 1.73 & 1.73 & 149.2 & 149.2 & 3.47 & 134.5 \\
\hline TACJOY & $P 2_{1} / \mathrm{c}$ & 1.74 & 1.72 & 161.1 & 101.6 & 3.47 & 61.2 \\
\hline TEXRUM & $P 21 / \mathrm{n}$ & 1.71 & 1.71 & 154.9 & 154.9 & 3.40 & 180.0 \\
\hline UDAJAL & $P 2_{1} / \mathrm{C}$ & 1.77 & 1.77 & 153.6 & 153.6 & 3.48 & -180.0 \\
\hline YEKZOG & $P 21 / \mathrm{n}$ & 1.71 & 1.72 & 125.1 & 123.2 & 3.42 & 132.6 \\
\hline YOMMIW & $P 2_{1} / \mathrm{C}$ & 1.74 & 1.74 & 147.7 & 147.7 & 3.38 & 180.0 \\
\hline ZOZZOG & $P-1$ & 1.73 & 1.75 & 163.1 & 156.8 & 3.33 & 123.5 \\
\hline
\end{tabular}

1.2. Chlorine-based halogen bonds

Table S4. Overview of CSD database entries that contain $\mathrm{Cl} \cdots \mathrm{O}$ halogen bonds.

\begin{tabular}{c|cccccc} 
Entry & Geometry & Space Group & $\mathbf{C}-\mathbf{C l}(\mathbf{A})$ & $\boldsymbol{\theta}_{\mathrm{c}-\mathrm{cl} \cdots \mathrm{O}}$ & \multicolumn{1}{c}{ c-o $\cdots \mathrm{cl}$} & $\mathbf{C l} \cdots \mathbf{O}(\mathbf{A})$ \\
\hline AGUYUW & $\mathrm{M}(\mathrm{Cl}) \cdots \beta(\mathrm{O})$ & $P-1$ & 1.74 & 171.8 & 128.6 & 3.04 \\
XAHMUO & $\mathrm{M}(\mathrm{Cl}) \cdots \beta(\mathrm{O})$ & $P-1$ & 1.67 & 154.3 & 130.0 & 3.22
\end{tabular}

Table S5. Overview of CSD database entries that contain $\mathrm{Cl} \cdots \mathrm{N}$ halogen bonds.

\begin{tabular}{l|cccccc}
\multicolumn{1}{c|}{ Entry } & Geometry & $\begin{array}{c}\text { Space } \\
\text { Group }\end{array}$ & $\mathbf{C}-\mathbf{C l}(\mathbf{A})$ & $\boldsymbol{\theta}_{\mathrm{C}-\mathrm{Cl} \cdots \mathbf{N}}$ & $\mathbf{C l} \cdots \mathbf{O}(\mathbf{A})$ & XB acceptor \\
\hline CERWEE & $\mathrm{A}(\mathrm{Cl}) \cdots \mathrm{M}(\mathrm{N})$ & $\mathrm{P} 21 / \mathrm{c}$ & 1.74 & 122.2 & 3.23 & nitrogen atom \\
IMEZUV & $\mathrm{M} \cdots$ Pyrrole & $\mathrm{P} 21 / \mathrm{c}$ & 1.74 & 161.3 & 3.09 & $\pi$-system \\
MULVUK & $\mathrm{M} \cdots$ Pyrrole & $\mathrm{C} 2 / \mathrm{c}$ & 1.70 & 164.2 & 3.06 & $\pi$-system \\
QUCZUK & $\mathrm{M} \cdots$ Pyrrole & $\mathrm{P} 2 / \mathrm{c}$ & 1.76 & 168.9 & 3.26 & $\pi$-system
\end{tabular}




\section{Figures and tables for bromine-based interactions}

\section{1. $\mathrm{Br} \cdots \mathrm{Br}$ interactions}

a)

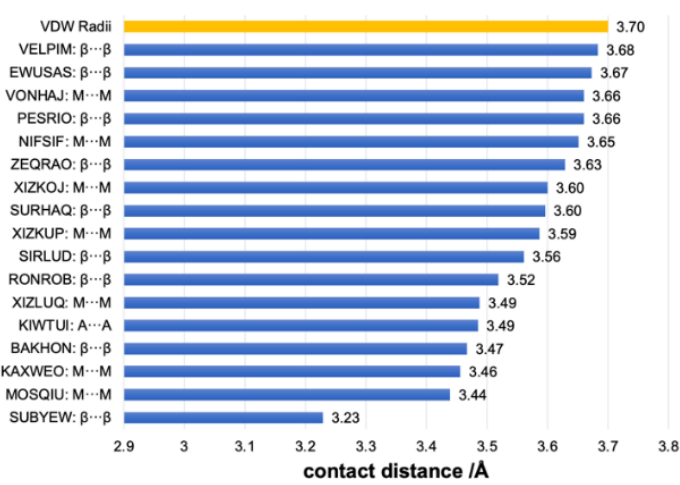

b)

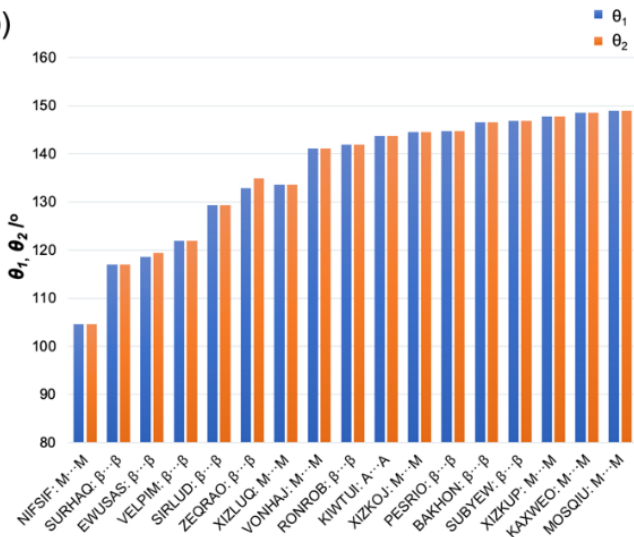

Figure S3. Histograms of geometrical parameters for Type I $\mathrm{Br} \cdots \mathrm{Br}$ interactions: a) interatomic distances and $b) \theta$ angles.

a)

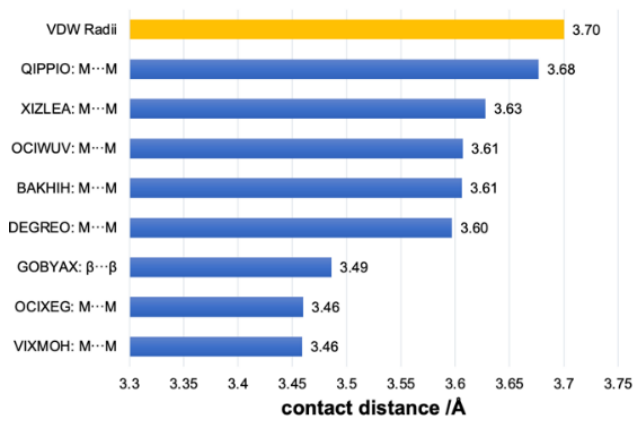

b)

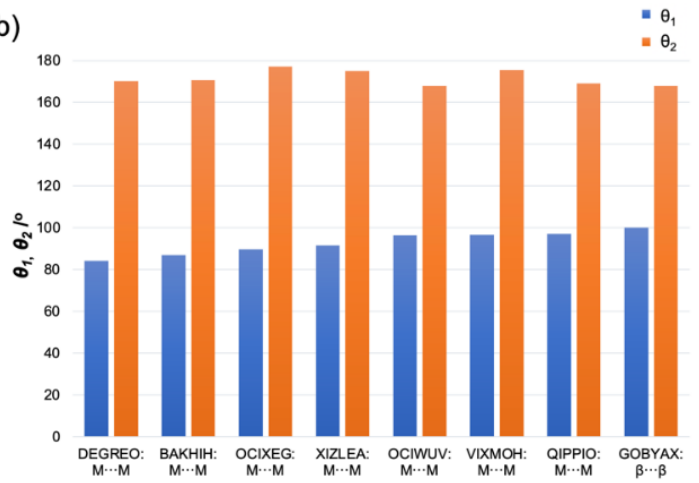

Figure S4. Histograms of geometrical parameters for Type II $\mathrm{Br} \cdots \mathrm{Br}$ interactions: a) interatomic distances and $b) \theta$ angles.

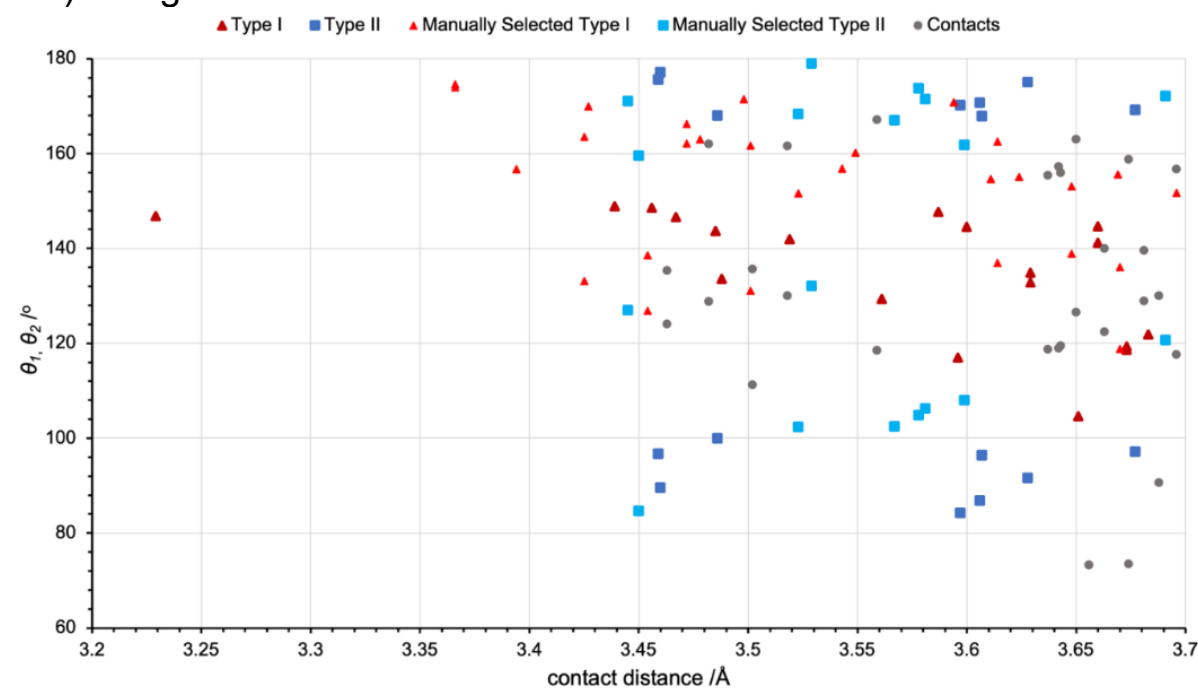

Figure S5. Scatterplot of interatomic distances vs $\theta$ angles for all $\mathrm{Br} \cdots \mathrm{Br}$ interactions or contacts. Type I and II interactions that were identified on visual inspection are shown in light red and cyan, respectively, while outliers that do not represent typical halogen $\cdots$ halogen interactions are represented by grey circles. 
Table S6. Overview of CSD database entries that contain Type I $\mathrm{Br} \cdots \mathrm{Br}$ interactions.

\begin{tabular}{|c|c|c|c|c|c|c|c|c|}
\hline Entry & Geometry & $\begin{array}{l}\text { Space } \\
\text { Group }\end{array}$ & $\mathrm{C}-\mathrm{Br}(\mathrm{A})$ & $\mathrm{C}-\mathrm{Br}(\mathrm{A})$ & $\theta_{1}\left({ }^{\circ}\right)$ & $\boldsymbol{\theta}_{2}\left({ }^{\circ}\right)$ & $\mathrm{Br} \cdots \mathrm{Br}(\mathrm{A})$ & $\varphi\left({ }^{\circ}\right)$ \\
\hline BAKHON & $\beta \cdots \beta$ & $14_{1} / a$ & 1.87 & 1.87 & 146.5 & 146.5 & 3.47 & -180.0 \\
\hline EWUSAS & $\beta \cdots \beta$ & $P-1$ & 1.88 & 1.87 & 118.6 & 119.4 & 3.67 & -123.8 \\
\hline KAXWEO & $M \cdots M$ & $P-1$ & 1.91 & 1.91 & 148.5 & 148.5 & 3.46 & 180.0 \\
\hline KIWTUI & $A \cdots A$ & $P-1$ & 1.88 & 1.88 & 143.7 & 143.7 & 3.49 & -180.0 \\
\hline MOSQIU & $M \cdots M$ & $P-1$ & 1.91 & 1.91 & 148.9 & 148.9 & 3.44 & 180.0 \\
\hline PESRIO & $\beta \cdots \beta$ & $P-1$ & 1.88 & 1.88 & 144.7 & 144.7 & 3.66 & 180.0 \\
\hline RONROB & $\beta \cdots \beta$ & $14_{1} / a$ & 1.88 & 1.88 & 141.9 & 141.9 & 3.52 & -180.0 \\
\hline SIRLUD & $\beta \cdots \beta$ & $14_{1} / a$ & 1.87 & 1.87 & 129.4 & 129.4 & 3.56 & 180.0 \\
\hline SURHAQ & $\beta \cdots \beta$ & $P-1$ & 1.86 & 1.86 & 116.9 & 116.9 & 3.60 & 180.0 \\
\hline VELPIM & $\beta \cdots \beta$ & $C 2 / c$ & 1.86 & 1.86 & 121.9 & 121.9 & 3.68 & 109.3 \\
\hline VONHAJ & $M \cdots M$ & $P-1$ & 1.90 & 1.90 & 141.2 & 141.2 & 3.66 & -180.0 \\
\hline XIZKOJ & $\mathrm{M} \cdots \mathrm{M}$ & $P-1$ & 1.90 & 1.90 & 144.5 & 144.5 & 3.60 & 180.0 \\
\hline XIZKUP & $M \cdots M$ & $P-1$ & 1.90 & 1.90 & 147.7 & 147.7 & 3.59 & -180.0 \\
\hline XIZLUQ & $M \cdots M$ & $P-1$ & 1.90 & 1.90 & 133.6 & 133.6 & 3.49 & 180.0 \\
\hline ZEQRAO & $\beta \cdots \beta$ & $P-1$ & 1.84 & 1.87 & 132.8 & 134.8 & 3.63 & 157.7 \\
\hline SUBYEW & $\beta \cdots \beta$ & $P 21 / \mathrm{n}$ & 1.87 & 1.87 & 146.9 & 146.9 & 3.23 & 180.0 \\
\hline NIFSIF & $M \cdots M$ & $P-1$ & 1.90 & 1.90 & 104.6 & 104.6 & 3.65 & 180.0 \\
\hline
\end{tabular}

Table S7. Overview of CSD database entries that contain Type II $\mathrm{Br} \cdots \mathrm{Br}$ interactions

\begin{tabular}{lcccccccc}
\multicolumn{1}{c}{ Entry } & Geometry & $\begin{array}{c}\text { Space } \\
\text { Group }\end{array}$ & $\mathbf{C}-\mathbf{B r}(\mathbf{A})$ & $\mathbf{C}-\mathbf{B r}(\mathbf{A})$ & $\boldsymbol{\theta}_{\mathbf{1}}\left(^{(}\right)$ & $\mathbf{\theta}_{\mathbf{2}}\left(^{\circ}\right)$ & $\mathbf{B r} \cdots \mathbf{B r}(\mathbf{A})$ & $\boldsymbol{\varphi}\left(^{(}\right)$ \\
BAKHIH & $\mathrm{M} \cdots \mathrm{M}$ & $P-1$ & 1.89 & 1.90 & 86.8 & 170.7 & 3.61 & 60.1 \\
DEGREO & $\mathrm{M} \cdots \mathrm{M}$ & $P 1$ & 1.90 & 1.90 & 84.2 & 170.2 & 3.60 & -82.5 \\
GOBYAX & $\beta \cdots \beta$ & $P 21 / \mathrm{n}$ & 1.88 & 1.88 & 100.0 & 168.0 & 3.49 & -177.8 \\
OCIWUV & $\mathrm{M} \cdots \mathrm{M}$ & $P 21 / \mathrm{c}$ & 1.89 & 1.90 & 96.3 & 167.9 & 3.61 & 140.8 \\
OCIXEG & $\mathrm{M} \cdots \mathrm{M}$ & $P-1$ & 1.90 & 1.89 & 89.6 & 177.0 & 3.46 & -173.9 \\
QIPPIO & $\mathrm{M} \cdots \mathrm{M}$ & $P-1$ & 1.89 & 1.90 & 97.1 & 169.1 & 3.68 & 49.4 \\
VIXMOH & $\mathrm{M} \cdots \mathrm{M}$ & $P$ cab & 1.90 & 1.93 & 96.7 & 175.6 & 3.46 & 162.5 \\
XIZLEA & $\mathrm{M} \cdots \mathrm{M}$ & $P-1$ & 1.90 & 1.89 & 91.6 & 175.0 & 3.63 & 97.4
\end{tabular}

Table S8. Overview of all CSD database entries that contain $\mathrm{Br} \cdots \mathrm{Br}$ interactions.

\begin{tabular}{|c|c|c|c|c|c|c|c|}
\hline Entry & $\begin{array}{l}\text { Space } \\
\text { Group }\end{array}$ & $\mathrm{C}-\mathrm{Br}(\mathrm{A})$ & $\mathrm{C}-\mathrm{Br}(\mathrm{A})$ & $\theta_{1}\left(^{\circ}\right)$ & $\theta_{2}\left({ }^{\circ}\right)$ & $\operatorname{Br} \cdots \operatorname{Br}(\AA)$ & $\varphi\left({ }^{\circ}\right)$ \\
\hline BAKHIH & $P-1$ & 1.89 & 1.90 & 86.8 & 170.7 & 3.61 & 60.1 \\
\hline BAKHON & $14_{1} / a$ & 1.87 & 1.87 & 146.5 & 146.5 & 3.47 & -180.0 \\
\hline BAKJAB & $C 2 / c$ & 1.85 & 1.89 & 135.6 & 111.2 & 3.50 & 153.6 \\
\hline BAKJAB & $C 2 / c$ & 1.90 & 1.86 & 126.6 & 163.0 & 3.65 & -164.4 \\
\hline COGWED & $P 2 / \mathrm{c}$ & 1.87 & 1.87 & 117.6 & 156.7 & 3.70 & 159.0 \\
\hline DEGREO & $P 1$ & 1.91 & 1.86 & 107.9 & 161.8 & 3.60 & -49.4 \\
\hline DEGREO & $P 1$ & 1.90 & 1.90 & 170.2 & 84.2 & 3.60 & -82.5 \\
\hline DUKGEX & $P-1$ & 1.91 & 1.91 & 156.7 & 156.7 & 3.39 & -180.0 \\
\hline EWURIZ & $P 21 / \mathrm{c}$ & 1.88 & 1.88 & 162.9 & 162.9 & 3.48 & 180.0 \\
\hline EWUROF & $P 2_{1} / \mathrm{m}$ & 1.88 & 1.88 & 73.2 & 73.2 & 3.66 & 180.0 \\
\hline EWUSAS & $P-1$ & 1.88 & 1.87 & 118.6 & 119.4 & 3.67 & -123.8 \\
\hline FEYMUS & $P 2_{1} / \mathrm{n}$ & 1.88 & 1.89 & 84.6 & 159.5 & 3.45 & -53.1 \\
\hline FEYMUS & $P 2_{1} / \mathrm{n}$ & 1.88 & 1.87 & 166.3 & 162.1 & 3.47 & 128.9 \\
\hline GOBYAX & $P 2_{1} / \mathrm{n}$ & 1.88 & 1.88 & 100.0 & 168.0 & 3.49 & -177.8 \\
\hline GOBYAX01 & $P 2{ }_{1}{ }_{1} 2_{1}$ & 1.89 & 1.89 & 178.9 & 132.0 & 3.53 & -144.4 \\
\hline GOGNIA & $P 2_{1} / \mathrm{n}$ & 1.86 & 1.86 & 170.8 & 170.8 & 3.59 & 180.0 \\
\hline HOXRIV & $P-1$ & 1.87 & 1.87 & 171.4 & 106.2 & 3.58 & -63.8 \\
\hline HUPFAZ & $P-1$ & 1.87 & 1.87 & 104.8 & 173.8 & 3.58 & -102.0 \\
\hline KAXWEO & $P-1$ & 1.91 & 1.91 & 148.5 & 148.5 & 3.46 & 180.0 \\
\hline
\end{tabular}




\begin{tabular}{|c|c|c|c|c|c|c|c|}
\hline KIWTUI & $P-1$ & 1.88 & 1.88 & 143.7 & 143.7 & 3.49 & -180.0 \\
\hline KIWVIY & $P-1$ & 1.89 & 1.89 & 155.6 & 155.6 & 3.67 & 180.0 \\
\hline LATSEE & $C 2 / c$ & 1.89 & 1.88 & 128.8 & 162.0 & 3.48 & -99.8 \\
\hline LATSEE & $C 2 / c$ & 1.86 & 1.87 & 157.2 & 118.9 & 3.64 & 121.3 \\
\hline LINPON & $P 21 / \mathrm{n}$ & 1.87 & 1.87 & 171.4 & 171.4 & 3.50 & -180.0 \\
\hline LINPON01 & $P 2{ }_{1} / \mathrm{n}$ & 1.87 & 1.87 & 170.0 & 170.0 & 3.43 & -180.0 \\
\hline LOPXOD & Pnma & 1.88 & 1.86 & 138.6 & 126.8 & 3.45 & 152.8 \\
\hline MOSQIU & $P-1$ & 1.90 & 1.90 & 104.6 & 104.6 & 3.65 & 180.0 \\
\hline NIFSIF & $P-1$ & 1.91 & 1.91 & 148.9 & 148.9 & 3.44 & 180.0 \\
\hline OCIWUV & $P 2_{1} / \mathrm{c}$ & 1.89 & 1.90 & 96.3 & 167.9 & 3.61 & 140.8 \\
\hline OCIXAC & $P-1$ & 1.90 & 1.90 & 168.3 & 102.4 & 3.52 & 126.6 \\
\hline OCIXEG & $P-1$ & 1.90 & 1.89 & 89.6 & 177.0 & 3.46 & -173.9 \\
\hline OCIXEG & $P-1$ & 1.90 & 1.90 & 156.8 & 156.8 & 3.54 & -180.0 \\
\hline OCIXEG & $P-1$ & 1.90 & 1.90 & 151.6 & 151.6 & 3.52 & 180.0 \\
\hline OKOSUC & $P-1$ & 1.90 & 1.90 & 139.5 & 128.9 & 3.68 & 35.7 \\
\hline РАHKOY & C2/c & 1.86 & 1.88 & 131.1 & 161.6 & 3.50 & -99.8 \\
\hline PAHKOY & C2/c & 1.87 & 1.87 & 155.4 & 118.7 & 3.64 & 123.9 \\
\hline РАНKOY01 & C2/c & 1.87 & 1.86 & 130.0 & 161.6 & 3.52 & -100.0 \\
\hline PAHKOY01 & $C 2 / c$ & 1.88 & 1.87 & 155.9 & 119.4 & 3.64 & 126.7 \\
\hline PESRIO & $P-1$ & 1.88 & 1.88 & 144.7 & 144.7 & 3.66 & 180.0 \\
\hline QIPPIO & $P-1$ & 1.90 & 1.89 & 169.1 & 97.1 & 3.68 & -49.4 \\
\hline RONROB & 141/a & 1.88 & 1.88 & 141.9 & 141.9 & 3.52 & -180.0 \\
\hline RUJPAN & $P-1$ & 1.86 & 1.87 & 118.5 & 167.1 & 3.56 & -110.1 \\
\hline RUJPAN & $P-1$ & 1.86 & 1.86 & 139.9 & 122.4 & 3.66 & 131.8 \\
\hline RUJPAN & $P-1$ & 1.87 & 1.87 & 130.0 & 90.6 & 3.69 & 138.2 \\
\hline SIRLUD & $14{ }_{1} / \mathrm{a}$ & 1.87 & 1.87 & 129.4 & 129.4 & 3.56 & 180.0 \\
\hline SURHAQ & $P-1$ & 1.86 & 1.86 & 116.9 & 116.9 & 3.60 & 180.0 \\
\hline TARJOO & $P-1$ & 1.90 & 1.90 & 120.7 & 172.1 & 3.69 & 59.0 \\
\hline VELPIM & $\mathrm{C} 2 / \mathrm{c}$ & 1.86 & 1.86 & 121.9 & 121.9 & 3.68 & 109.3 \\
\hline VIXMOH & Pcab & 1.90 & 1.91 & 174.0 & 174.6 & 3.37 & 10.2 \\
\hline VIXMOH & Pcab & 1.90 & 1.93 & 96.7 & 175.6 & 3.46 & 162.5 \\
\hline VIXMOH & Pcab & 1.80 & 1.87 & 171.0 & 127.0 & 3.45 & 158.9 \\
\hline VONHAJ & $P-1$ & 1.90 & 1.90 & 141.2 & 141.2 & 3.66 & -180.0 \\
\hline WULDOY & $P-1$ & 1.88 & 1.90 & 138.9 & 153.1 & 3.65 & 170.1 \\
\hline WUTRUZ & $P 2{ }_{1} / \mathrm{n}$ & 1.90 & 1.90 & 154.6 & 154.6 & 3.61 & -180.0 \\
\hline XIZKOJ & $P-1$ & 1.90 & 1.90 & 144.5 & 144.5 & 3.60 & 180.0 \\
\hline XIZKUP & $P-1$ & 1.90 & 1.90 & 147.7 & 147.7 & 3.59 & -180.0 \\
\hline XIZLEA & $P-1$ & 1.89 & 1.90 & 175.0 & 91.6 & 3.63 & -97.4 \\
\hline XIZLIE & $P-1$ & 1.91 & 1.90 & 167.0 & 102.4 & 3.57 & -36.1 \\
\hline XIZLIE & $P-1$ & 1.90 & 1.90 & 160.1 & 160.1 & 3.55 & -180.0 \\
\hline XIZLOK & $P 2_{1} / \mathrm{n}$ & 1.90 & 1.89 & 73.5 & 158.8 & 3.67 & 179.0 \\
\hline XIZLUQ & $P-1$ & 1.90 & 1.90 & 133.6 & 133.6 & 3.49 & 180.0 \\
\hline YAVBOO & Aba2 & 1.88 & 1.88 & 135.4 & 124.0 & 3.46 & -79.3 \\
\hline ZALJEB & C2 & 1.86 & 1.86 & 155.0 & 155.0 & 3.62 & 23.4 \\
\hline ZEQQUH & $P-1$ & 1.86 & 1.82 & 136.9 & 162.6 & 3.61 & -74.9 \\
\hline ZEQQUH & $P-1$ & 1.88 & 1.87 & 133.2 & 163.5 & 3.43 & 87.1 \\
\hline ZEQRAO & $P-1$ & 1.85 & 1.86 & 118.9 & 136.0 & 3.67 & 113.1 \\
\hline ZEQRAO & $P-1$ & 1.84 & 1.87 & 132.8 & 134.8 & 3.63 & 157.7 \\
\hline SUBYEW & $P 2_{1} / \mathrm{n}$ & 1.87 & 1.87 & 146.9 & 146.9 & 3.23 & 180.0 \\
\hline ZOZZIA & $P-1$ & 2.10 & 2.10 & 151.7 & 151.7 & 3.70 & 180.0 \\
\hline
\end{tabular}




\subsection{Bromine-based halogen bonds}

a)

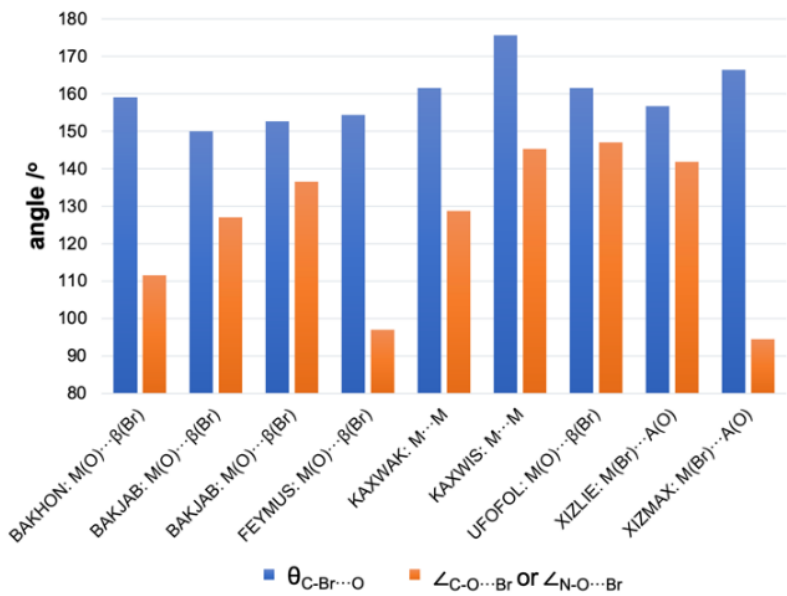

b)

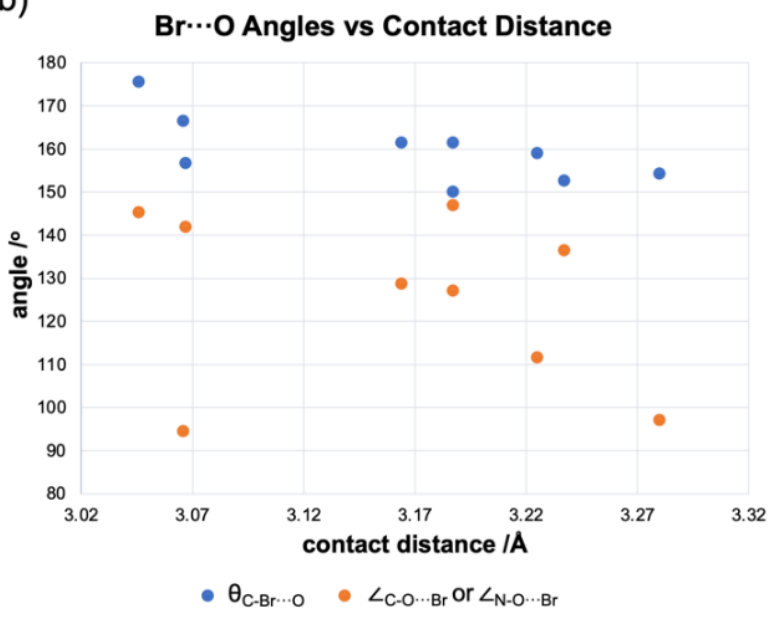

Figure S6. a) Histograms of $\mathrm{C}-\mathrm{Br} \cdots \mathrm{O}$ and $\mathrm{Br} \cdots \mathrm{O}-\mathrm{C}$ or $\mathrm{Br} \cdots \mathrm{O}-\mathrm{N}$ angles and $\mathrm{b}$ ) scatterplot of interatomic distance vs the $\mathrm{C}-\mathrm{Br} \cdots \mathrm{O}, \mathrm{Br} \cdots \mathrm{O}-\mathrm{C}$ or $\mathrm{Br} \cdots \mathrm{O}-\mathrm{N}$ angle for structures exhibiting $\mathrm{Br} \cdots \mathrm{O}$ halogen bonds and contacts.

a)

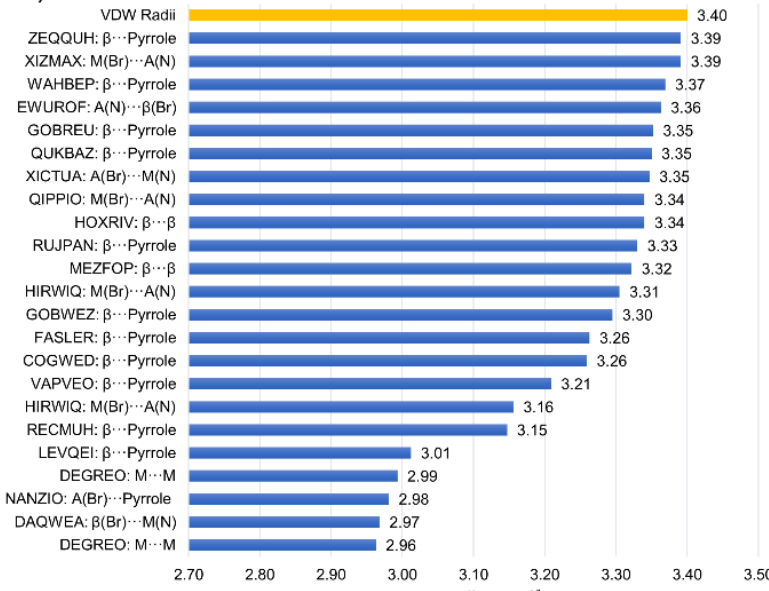

b)

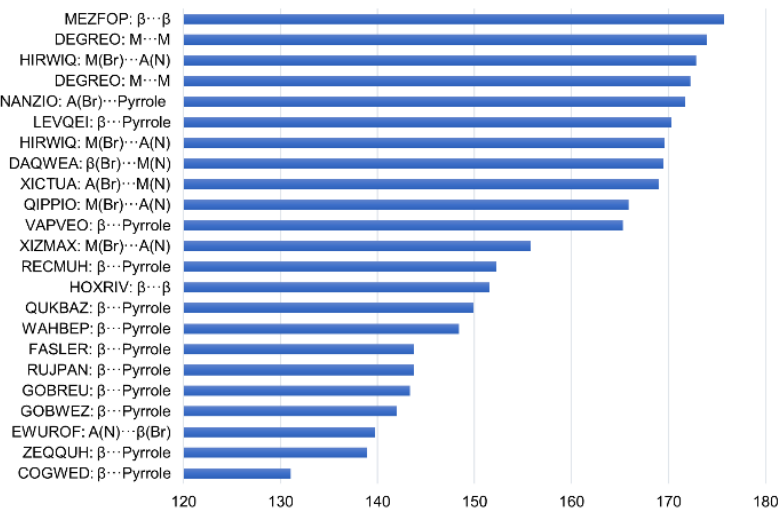

Figure S7. Histograms of C-Br $\cdots \mathrm{N}$ interaction properties: a) interatomic distances and b) $\theta$ angles.

Table S9. Overview of CSD entries that contain $\mathrm{Br} \cdots \mathrm{O}$ interactions.

\begin{tabular}{|c|c|c|c|c|c|c|}
\hline Entry & Geometry & Space Group & $\theta_{\mathrm{C}-\mathrm{Br} \cdots \mathrm{O}}\left({ }^{\circ}\right)$ & $\begin{array}{l}\angle \mathrm{C}-\mathrm{O} \cdots \mathrm{Br} \text { Or } \\
\angle \mathrm{N}-\mathrm{O} \cdots \mathrm{Br}\left({ }^{\circ}\right)\end{array}$ & $\mathrm{C}-\mathrm{Br}(\mathrm{A})$ & $\mathrm{Br} \cdots \mathrm{O}(\mathrm{A})$ \\
\hline BAKHON & $\mathrm{M}(\mathrm{O}) \cdots \beta(\mathrm{Br})$ & $141 / a$ & 159.0 & 111.6 & 1.87 & 3.23 \\
\hline BAKJAB & $M(O) \cdots \beta(B r)$ & $C 2 / \mathrm{c}$ & 150.0 & 127.1 & 1.85 & 3.19 \\
\hline BAKJAB & $M(O) \cdots \beta(B r)$ & $C 2 / c$ & 152.6 & 136.5 & 1.85 & 3.24 \\
\hline FEYMUS & $M(O) \cdots \beta(B r)$ & $P 21_{1} / \mathrm{n}$ & 154.3 & 97.0 & 1.88 & 3.28 \\
\hline KAXWAK & $M \cdots M$ & $P-1$ & 161.5 & 128.7 & 1.91 & 3.16 \\
\hline KAXWIS & $\mathrm{M} \cdots \mathrm{M}$ & $C 2 / c$ & 175.6 & 145.3 & 1.91 & 3.05 \\
\hline UFOFOL & $M(O) \cdots \beta(B r)$ & $C 2 / c$ & 161.5 & 147.0 & 1.88 & 3.19 \\
\hline XIZLIE & $M(B r) \cdots A(O)$ & $P-1$ & 156.8 & 141.9 & 1.90 & 3.07 \\
\hline XIZMAX & $M(B r) \cdots A(O)$ & $C 2 / c$ & 166.4 & 94.4 & 1.90 & 3.07 \\
\hline
\end{tabular}


Table S10. Overview of CSD entries that contain $\mathrm{Br} \cdots \mathrm{N}$ interactions.

\begin{tabular}{|c|c|c|c|c|c|c|}
\hline Name & Geometry & $\begin{array}{l}\text { Space } \\
\text { Group }\end{array}$ & $\boldsymbol{\theta}\left({ }^{\circ}\right)$ & $\mathrm{C}-\mathrm{Br}(\mathrm{A})$ & $\mathrm{Br} \cdots \mathrm{N}(\AA)$ & XB acceptor \\
\hline COGWED & $\beta \cdots$ Pyrrole & $P 2 / \mathrm{c}$ & 131.1 & 1.88 & 3.26 & $\pi$-system \\
\hline DAQWEA & $\beta(\mathrm{Br}) \cdots \mathrm{M}(\mathrm{N})$ & $P-1$ & 169.5 & 1.86 & 2.97 & $\pi$-system \\
\hline DEGREO & $M \cdots M$ & $P 1$ & 172.3 & 1.90 & 2.96 & $\mathrm{~N}$-atom lone pair \\
\hline DEGREO & $M \cdots M$ & $P 1$ & 174.0 & 1.91 & 2.99 & $\mathrm{~N}$-atom lone pair \\
\hline EWUROF & $A(N) \cdots \beta(B r)$ & $P 21 / \mathrm{m}$ & 139.7 & 1.88 & 3.36 & $\pi$-system \\
\hline FASLER & $\beta \cdots$ Pyrrole & $P-1$ & 143.8 & 1.88 & 3.26 & $\pi$-system \\
\hline GOBREU & $\beta \cdots$ Pyrrole & $P-1$ & 143.4 & 1.88 & 3.35 & $\pi$-system \\
\hline GOBWEZ & $\beta \cdots$ Pyrrole & $P-1$ & 142.0 & 1.87 & 3.30 & $\pi$-system \\
\hline HIRWIQ & $\mathrm{M}(\mathrm{Br}) \cdots \mathrm{A}(\mathrm{N})$ & $P 2_{1} / \mathrm{c}$ & 169.6 & 1.91 & 3.31 & $\mathrm{~N}$-atom lone pair \\
\hline HIRWIQ & $M(B r) \cdots A(N)$ & $P 2_{1} / \mathrm{c}$ & 172.9 & 1.91 & 3.16 & $\mathrm{~N}$-atom lone pair \\
\hline HOXRIV & $\beta \cdots \beta$ & $P-1$ & 151.5 & 1.87 & 3.34 & $\pi$-system \\
\hline LEVQEI & $\beta \cdots$ Pyrrole & $P 2_{1} / \mathrm{c}$ & 170.3 & 1.86 & 3.01 & $\pi$-system \\
\hline MEZFOP & $\beta \cdots \beta$ & $P 2_{1} / \mathrm{n}$ & 175.7 & 1.87 & 3.32 & $\pi$-system \\
\hline NANZIO & A $\cdots$ Pyrrole & $P 212121$ & 171.7 & 1.87 & 2.98 & $\pi$-system \\
\hline QIPPIO & $M(B r) \cdots A(N)$ & $P-1$ & 165.9 & 1.89 & 3.34 & $\pi$-system \\
\hline QUKBAZ & $\beta \cdots$ Pyrrole & $P 2_{1} / \mathrm{m}$ & 149.9 & 1.87 & 3.35 & $\pi$-system \\
\hline RECMUH & $\beta \cdots$ Pyrrole & $R-3$ & 152.2 & 1.87 & 3.15 & $\pi$-system \\
\hline RUJPAN & $\beta \cdots$ Pyrrole & $P-1$ & 143.7 & 1.88 & 3.33 & $\pi$-system \\
\hline VAPVEO & $\beta \cdots$ Pyrrole & Pcab & 165.3 & 1.81 & 3.21 & $\pi$-system \\
\hline WAHBEP & $\beta \cdots$ Pyrrole & R-3 & 148.4 & 1.88 & 3.37 & $\pi$-system \\
\hline XICTUA & $\mathrm{A}(\mathrm{Br}) \cdots \mathrm{M}(\mathrm{N})$ & $C 2 / c$ & 169.0 & 1.90 & 3.35 & $\mathrm{~N}$-atom lone pair \\
\hline ZEQQUH & $\beta \cdots$ Pyrrole & $P-1$ & 155.8 & 1.90 & 3.39 & $\pi$-system \\
\hline
\end{tabular}

\section{Figures and tables for iodine-based interactions}

\section{1. $1 \cdots$ I interactions}

a)

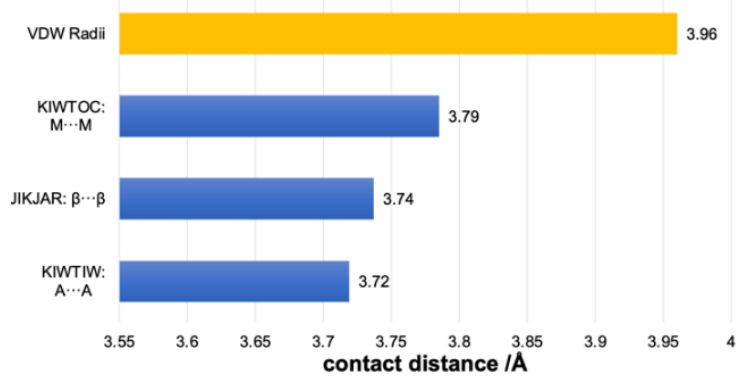

b)

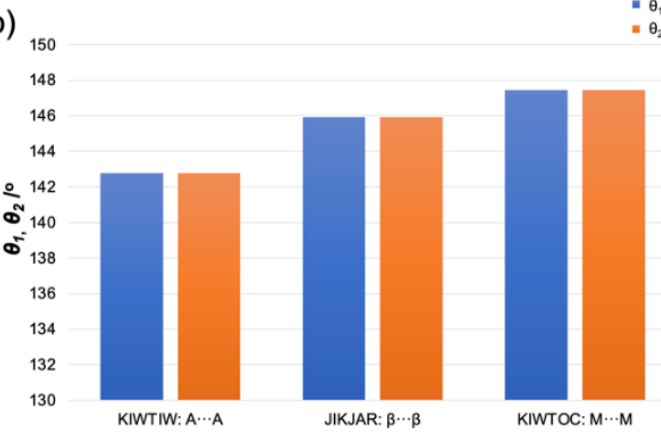

Figure S8. Histograms for Type I | $\cdots$ | interactions: a) interatomic distances and b) $\theta$ angles. 
a)

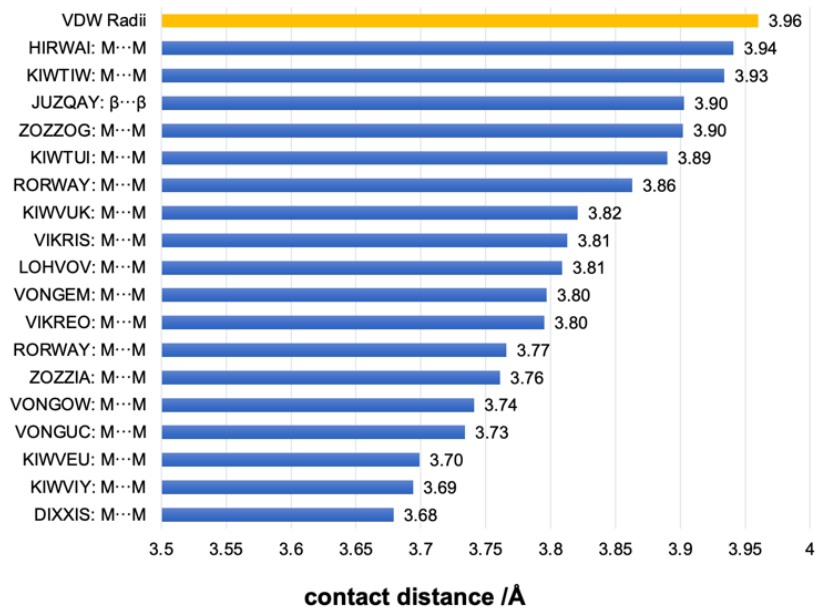

b)

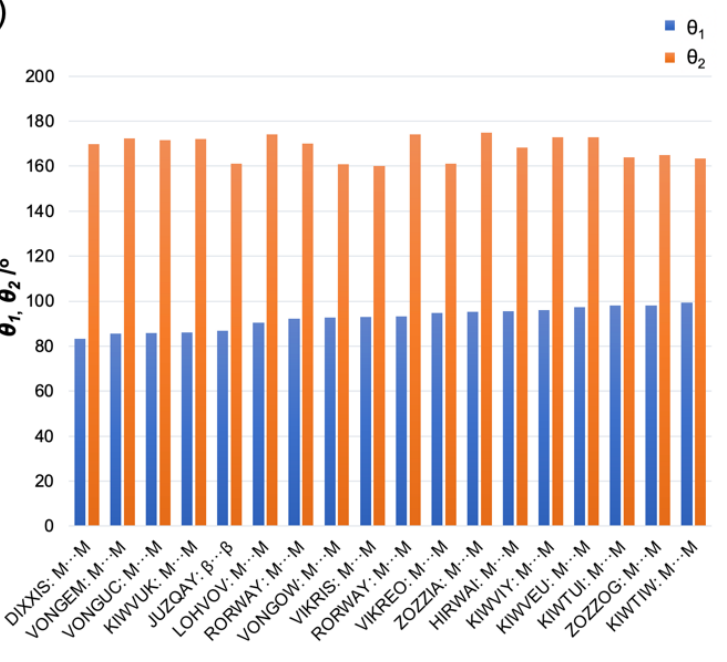

Figure S9. Histograms for Type II | $\cdots \mid$ interactions: a) interatomic distances and b) $\theta$ angles.

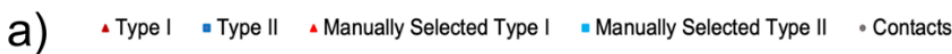

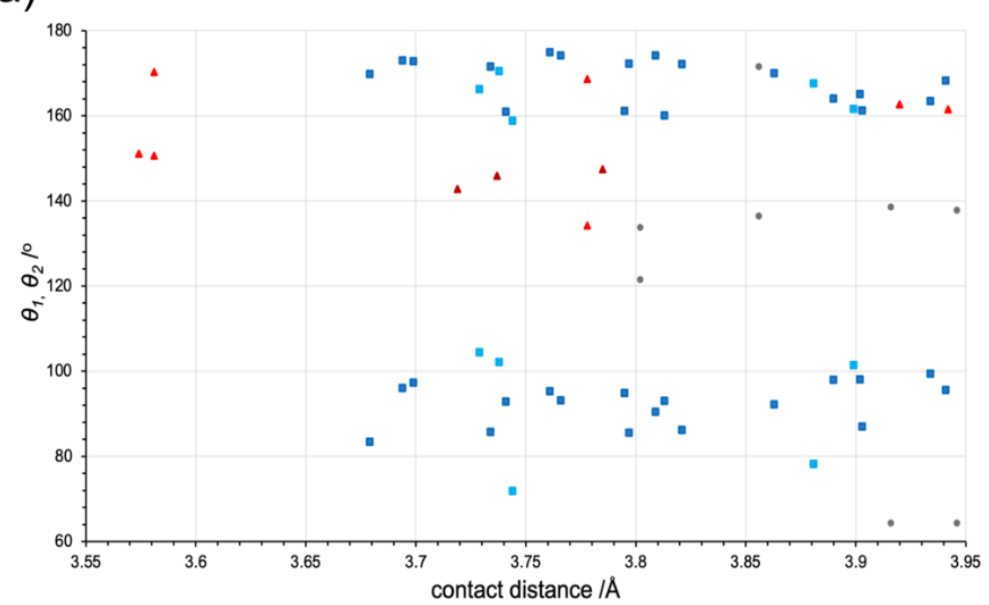

b)

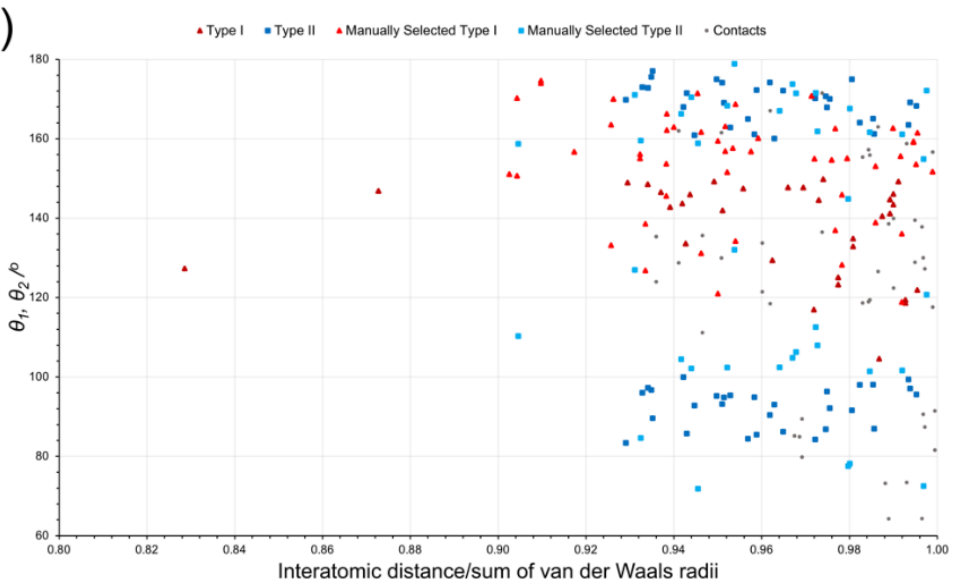

Figure S10. a) Scatterplot of interatomic distances vs $\theta$ angles for all $|\cdots|$ interactions and contacts. Type I and II interactions identified by visual inspection are shown in light red and cyan, respectively. Outliers that do not represent typical halogen $\cdots$ halogen interactions are represented by grey circles. b) Scatterplot of $\theta$-angles with respect to interatomic distances, expressed as the ratio of the distance to the sum of the van der Waals radii for the involved atoms. 
Table S11. Overview of CSD database entries that contain Type | $|\cdots|$ interactions.

\begin{tabular}{|c|c|c|c|c|c|c|c|c|}
\hline Entry & Geometry & $\begin{array}{l}\text { Space } \\
\text { Group }\end{array}$ & C-I (Å) & C-I $(\AA)$ & $\theta_{1}\left(^{\circ}\right)$ & $\theta_{2}\left({ }^{\circ}\right)$ & $|\cdots|(\AA)$ & $\varphi\left({ }^{\circ}\right)$ \\
\hline JIKJAR & $\beta \cdots \beta$ & $P-1$ & 2.07 & 2.07 & 145.9 & 145.9 & 3.74 & 180.0 \\
\hline KIWTIW & $A \cdots A$ & $P-1$ & 2.10 & 2.10 & 142.8 & 142.8 & 3.72 & -180.0 \\
\hline KIWTOC & $\mathrm{M} \cdots \mathrm{M}$ & $P-1$ & 2.11 & 2.11 & 147.4 & 147.4 & 3.79 & 180.0 \\
\hline
\end{tabular}

Table S12. Overview of CSD database entries that contain Type II $|\cdots|$ interactions.

\begin{tabular}{|c|c|c|c|c|c|c|c|c|}
\hline Entry & Geometry & $\begin{array}{l}\text { Space } \\
\text { Group }\end{array}$ & C-I (Å) & C-I (Å) & $\theta_{1}\left({ }^{\circ}\right)$ & $\theta_{2}\left(^{\circ}\right)$ & $|\cdots|(\AA)$ & $\varphi\left({ }^{\circ}\right)$ \\
\hline DIXXIS & $\mathrm{M} \cdots \mathrm{M}$ & $P 1$ & 2.10 & 2.12 & 83.4 & 169.8 & 3.68 & 75.2 \\
\hline HIRWAI & $M \cdots M$ & $\mathrm{C} 2 / \mathrm{c}$ & 2.12 & 2.12 & 95.6 & 168.3 & 3.94 & -62.4 \\
\hline JUZQAY & $\beta \cdots \beta$ & $P 2_{1} / \mathrm{c}$ & 2.06 & 2.08 & 86.9 & 161.2 & 3.90 & -42.0 \\
\hline KIWTIW & $M \cdots M$ & $P-1$ & 2.10 & 2.10 & 99.4 & 163.5 & 3.93 & 10.8 \\
\hline KIWTUI & $M \cdots M$ & $P-1$ & 2.08 & 2.09 & 98.0 & 164.1 & 3.89 & 0.0 \\
\hline KIWVEU & $M \cdots M$ & $P-1$ & 2.12 & 2.10 & 97.2 & 172.8 & 3.70 & -21.8 \\
\hline KIWVIY & $M \cdots M$ & $P-1$ & 2.12 & 2.13 & 96.0 & 173.0 & 3.69 & -17.9 \\
\hline KIWVUK & $\mathrm{M} \cdots \mathrm{M}$ & $P-1$ & 2.09 & 2.09 & 86.2 & 172.1 & 3.82 & 132.1 \\
\hline LOHVOV & $M \cdots M$ & $P 1$ & 2.11 & 2.11 & 90.4 & 174.2 & 3.81 & 137.5 \\
\hline RORWAY & $M \cdots M$ & $P 2 / \mathrm{C}$ & 2.14 & 2.06 & 92.1 & 170.0 & 3.86 & -81.8 \\
\hline RORWAY & $M \cdots M$ & $P 2 / \mathrm{C}$ & 2.13 & 2.10 & 93.2 & 174.1 & 3.77 & 104.6 \\
\hline VIKREO & $\mathrm{M} \cdots \mathrm{M}$ & $P 2{ }_{1} / \mathrm{c}$ & 2.11 & 2.11 & 94.9 & 161.1 & 3.80 & 66.3 \\
\hline VIKRIS & $M \cdots M$ & $P 2{ }_{1} / \mathrm{c}$ & 2.10 & 2.10 & 93.0 & 160.0 & 3.81 & -65.9 \\
\hline VONGEM & $M \cdots M$ & $C 2 / c$ & 2.11 & 2.11 & 85.5 & 172.3 & 3.80 & -127.6 \\
\hline VONGOW & $M \cdots M$ & $\mathrm{C} 2 / \mathrm{c}$ & 2.11 & 2.07 & 92.8 & 160.9 & 3.74 & -14.2 \\
\hline VONGUC & $\mathrm{M} \cdots \mathrm{M}$ & $C 2 / c$ & 2.10 & 2.12 & 85.7 & 171.5 & 3.73 & 0.0 \\
\hline ZOZZIA & $\mathrm{M} \cdots \mathrm{M}$ & $P-1$ & 2.08 & 2.09 & 95.2 & 175.0 & 3.76 & -3.6 \\
\hline ZOZZOG & $M \cdots M$ & $P-1$ & 2.10 & 2.11 & 98.0 & 165.1 & 3.90 & -5.9 \\
\hline
\end{tabular}

Table S13. Overview of all CSD database entries that contain $|\cdots|$ interactions and contacts.

\begin{tabular}{|c|c|c|c|c|c|c|c|}
\hline Entry & $\begin{array}{l}\text { Space } \\
\text { Group }\end{array}$ & C-I (Å) & C-I (A) & $\theta_{1}\left({ }^{\circ}\right)$ & $\theta_{2}\left(^{\circ}\right)$ & $|\cdots|(\AA)$ & $\varphi\left({ }^{\circ}\right)$ \\
\hline DIXXIS & $P 1$ & 2.10 & 2.12 & 83.4 & 169.8 & 3.68 & 75.2 \\
\hline DIXXIS & $P 1$ & 2.08 & 2.09 & 104.4 & 166.3 & 3.73 & 62.3 \\
\hline DIXXIS & $P 1$ & 2.13 & 2.08 & 137.8 & 64.3 & 3.95 & -157.2 \\
\hline HIRVOV & $P 2{ }_{1} / \mathrm{C}$ & 2.12 & 2.11 & 121.5 & 133.7 & 3.80 & 4.3 \\
\hline HIRVOV & $P 2_{1} / \mathrm{c}$ & 2.12 & 2.11 & 64.3 & 138.6 & 3.92 & 173.7 \\
\hline HIRVUB & $P 2_{1} / \mathrm{c}$ & 2.11 & 2.14 & 171.5 & 136.5 & 3.86 & 72.7 \\
\hline HIRWAI & C2/c & 2.12 & 2.12 & 168.3 & 95.6 & 3.94 & -62.4 \\
\hline JIKJAR & $P-1$ & 2.07 & 2.07 & 145.9 & 145.9 & 3.74 & 180.0 \\
\hline JUZQAY & $P 2{ }_{1} / \mathrm{C}$ & 2.06 & 2.08 & 86.9 & 161.2 & 3.90 & -42.0 \\
\hline JUZQEC & $P-1$ & 2.07 & 2.08 & 134.2 & 168.7 & 3.78 & -87.0 \\
\hline JUZQIG & $P-1$ & 2.09 & 2.10 & 170.2 & 150.6 & 3.58 & -159.3 \\
\hline KIWTIW & $P-1$ & 2.10 & 2.10 & 99.4 & 163.5 & 3.93 & 10.8 \\
\hline KIWTIW & $P-1$ & 2.10 & 2.10 & 142.8 & 142.8 & 3.72 & -180.0 \\
\hline KIWTOC & $P-1$ & 2.11 & 2.11 & 147.4 & 147.4 & 3.79 & 180.0 \\
\hline KIWTUI & $P-1$ & 2.09 & 2.08 & 164.1 & 98.0 & 3.89 & 0.0 \\
\hline KIWVEU & $P-1$ & 2.12 & 2.10 & 97.2 & 172.8 & 3.70 & -21.8 \\
\hline KIWVIY & $P-1$ & 2.12 & 2.13 & 96.0 & 173.0 & 3.69 & -17.9 \\
\hline KIWVOE & $P 2_{1} / \mathrm{c}$ & 2.12 & 2.10 & 101.4 & 161.6 & 3.90 & 61.5 \\
\hline KIWVUK & $P-1$ & 2.09 & 2.09 & 86.2 & 172.1 & 3.82 & 132.1 \\
\hline LOHVOV & $P 1$ & 2.11 & 2.11 & 174.2 & 90.4 & 3.81 & 137.5 \\
\hline
\end{tabular}




\begin{tabular}{l|ccccccc} 
LUGMOR & $C 2 / \mathrm{c}$ & 2.11 & 2.12 & 102.1 & 170.5 & 3.74 & -10.0 \\
RIGFAP & $P-1$ & 2.11 & 2.11 & 78.2 & 167.6 & 3.88 & 157.6 \\
RORWAY & $P 2 / \mathrm{c}$ & 2.14 & 2.06 & 92.1 & 170.0 & 3.86 & -81.8 \\
RORWAY & $P 2 / \mathrm{c}$ & 2.13 & 2.10 & 93.2 & 174.1 & 3.77 & 104.6 \\
SOCTUC & $P-1$ & 2.06 & 2.06 & 151.0 & 151.0 & 3.57 & -180.0 \\
VIKREO & $P 21 / \mathrm{c}$ & 2.11 & 2.11 & 161.1 & 94.9 & 3.80 & -66.3 \\
VIKRIS & $P 21 / \mathrm{c}$ & 2.10 & 2.10 & 160.0 & 93.0 & 3.81 & 65.9 \\
VONGEM & $\mathrm{C} 2 / \mathrm{c}$ & 2.11 & 2.11 & 85.5 & 172.3 & 3.80 & -127.6 \\
VONGOW & $\mathrm{C} / \mathrm{c}$ & 2.10 & 2.10 & 162.7 & 162.7 & 3.92 & 118.5 \\
VONGOW & $\mathrm{C} 2 / \mathrm{c}$ & 2.07 & 2.11 & 160.9 & 92.8 & 3.74 & 14.2 \\
VONGOW & $\mathrm{C} 2 / \mathrm{c}$ & 2.07 & 2.07 & 71.8 & 158.8 & 3.74 & 26.0 \\
VONGUC & $\mathrm{C} 2 / \mathrm{c}$ & 2.11 & 2.11 & 161.5 & 161.5 & 3.94 & 118.5 \\
VONGUC & $\mathrm{C} / \mathrm{c}$ & 2.10 & 2.12 & 85.7 & 171.5 & 3.73 & 0.0 \\
ZOZZIA & $P-1$ & 2.09 & 2.08 & 175.0 & 95.2 & 3.76 & -3.6 \\
ZOZZOG & $P-1$ & 2.10 & 2.11 & 98.0 & 165.1 & 3.90 & -5.9
\end{tabular}

\section{2. lodine-based halogen bonding interactions}
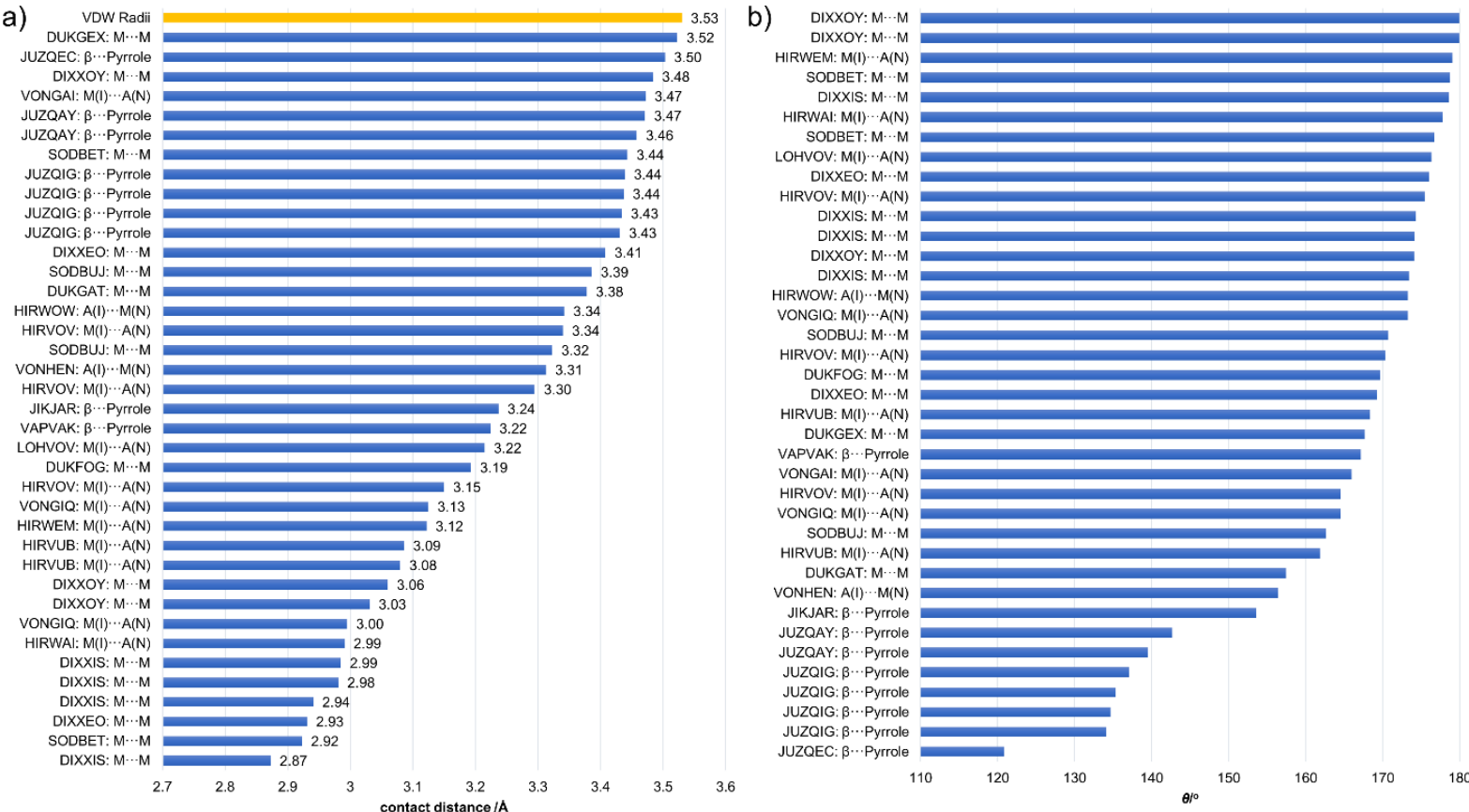

Figure S11. Histograms for $\mathrm{C}-\mathrm{I} \cdots \mathrm{N}$ interactions: a) interatomic distances and b) $\theta$ angles.

Table S14. Overview of CSD database entries that contain $1 \cdots O$ halogen bonds.

\begin{tabular}{|c|c|c|c|c|c|c|}
\hline Entry & Geometry & $\begin{array}{l}\text { Space } \\
\text { Group }\end{array}$ & $\theta_{\mathrm{C}-1 \cdots \mathrm{O}}\left({ }^{\circ}\right)$ & $\begin{array}{l}\angle \mathrm{C}-\mathrm{O} \cdots 1 \text { or } \\
\angle \mathrm{M}-\mathrm{O} \cdots \mathrm{O}\left({ }^{\circ}\right)\end{array}$ & $\mathrm{I} \cdots \mathrm{O}(\AA)$ & C-I (Å) \\
\hline AGOKOY & $M \cdots M$ & $P-1$ & 171.2 & 142.3 & 2.92 & 2.09 \\
\hline DUKGOH & $M(I) \cdots A(O)$ & $P-1$ & 158.4 & 117.2 & 3.26 & 2.10 \\
\hline DUKGUN & $M(I) \cdots A(O)$ & $P-1$ & 157.5 & 107.6 & 3.10 & 2.08 \\
\hline IFEBUT & $M \cdots M$ & $P-1$ & 142.1 & 87.3 & 3.45 & 2.10 \\
\hline KIWVEU & $M(I) \cdots A(O)$ & $P-1$ & 162.1 & 145.1 & 2.98 & 2.12 \\
\hline KIWVIY & $M(I) \cdots A(O)$ & $P-1$ & 162.2 & 144.9 & 3.00 & 2.12 \\
\hline LOHVOV & $M(I) \cdots A(O)$ & $P 1$ & 175.9 & 111.5 & 3.00 & 2.11 \\
\hline LUGMOR & $M(I) \cdots A(O)$ & C2/c & 168.9 & 113.4 & 2.95 & 2.11 \\
\hline VONFOV & $M(I) \cdots A(O)$ & $P-1$ & 154.0 & 105.7 & 3.30 & 2.10 \\
\hline VONGAI & $M(I) \cdots A(O)$ & $C 2 / c$ & 153.4 & 121.4 & 3.23 & 2.11 \\
\hline
\end{tabular}




\begin{tabular}{l|cccccr} 
VONGEM & $\mathrm{M}(\mathrm{I}) \cdots \mathrm{A}(\mathrm{O})$ & $\mathrm{C} 2 / \mathrm{c}$ & 159.0 & 123.3 & 3.13 & 2.10 \\
YENFUU & $\mathrm{M} \cdots \mathrm{A}$ & $P-1$ & 171.7 & 96.6 & 3.18 & 2.11 \\
ZOZZIA & $\mathrm{M}(\mathrm{I}) \cdots \mathrm{A}(\mathrm{O})$ & $P-1$ & 159.9 & 144.1 & 3.06 & 2.08
\end{tabular}

Table S15. Overview of CSD database entries that contain $\mathrm{I} \cdots \mathrm{N}$ halogen bonds.

\begin{tabular}{|c|c|c|c|c|c|c|}
\hline Entry & Geometry & $\begin{array}{l}\text { Space } \\
\text { Group }\end{array}$ & $C-I(A)$ & $\theta_{C-1 \cdots N}$ & $\mid \cdots N(\AA)$ & XB acceptor \\
\hline DIXXEO & $M \cdots M$ & $P 1$ & 2.12 & 176.0 & 2.93 & $\mathrm{~N}$-atom lone pair \\
\hline DIXXEO & $\mathrm{M} \cdots \mathrm{M}$ & $P 1$ & 2.09 & 169.3 & 3.41 & $\pi$-system \\
\hline DIXXIS & $\mathrm{M} \cdots \mathrm{M}$ & $P 1$ & 2.08 & 173.4 & 2.99 & $\mathrm{~N}$-atom lone pair \\
\hline DIXXIS & $\mathrm{M} \cdots \mathrm{M}$ & $P 1$ & 2.10 & 174.1 & 2.94 & $\mathrm{~N}$-atom lone pair \\
\hline DIXXIS & $M \cdots M$ & $P 1$ & 2.06 & 174.3 & 2.98 & $\mathrm{~N}$-atom lone pair \\
\hline DIXXIS & $\mathrm{M} \cdots \mathrm{M}$ & $P 1$ & 2.08 & 178.6 & 2.87 & $\mathrm{~N}$-atom lone pair \\
\hline DIXXOY & $\mathrm{M} \cdots \mathrm{M}$ & $C 2$ & 2.07 & 180.0 & 3.03 & $\mathrm{~N}$-atom lone pair \\
\hline DIXXOY & $M \cdots M$ & $C 2$ & 2.09 & 180.0 & 3.06 & $\mathrm{~N}$-atom lone pair \\
\hline DIXXOY & $M \cdots M$ & $C 2$ & 2.11 & 174.1 & 3.48 & $\pi$-system \\
\hline DUKFOG & $M \cdots M$ & $P-1$ & 2.07 & 169.7 & 3.19 & $\mathrm{~N}$-atom lone pair \\
\hline DUKGAT & $\mathrm{M} \cdots \mathrm{M}$ & $P 2_{1} / \mathrm{n}$ & 2.10 & 157.4 & 3.38 & $\pi$-system \\
\hline DUKGEX & $\mathrm{M} \cdots \mathrm{M}$ & $P-1$ & 2.10 & 167.7 & 3.52 & $\pi$-system \\
\hline HIRVOV & $M(I) \cdots A(N)$ & $P 2_{1} / \mathrm{c}$ & 2.12 & 175.4 & 3.15 & $\mathrm{~N}$-atom lone pair \\
\hline HIRVOV & $M(I) \cdots A(N)$ & $P 2_{1} / \mathrm{c}$ & 2.11 & 170.3 & 3.30 & $\mathrm{~N}$-atom lone pair \\
\hline HIRVOV & $M(I) \cdots A(N)$ & $P 2_{1} / \mathrm{C}$ & 2.12 & 164.6 & 3.34 & $\mathrm{~N}$-atom lone pair \\
\hline HIRVUB & $M(I) \cdots A(N)$ & $P 2_{1} / \mathrm{c}$ & 2.11 & 161.9 & 3.09 & $\mathrm{~N}$-atom lone pair \\
\hline HIRVUB & $M(I) \cdots A(N)$ & $P 2_{1} / \mathrm{c}$ & 2.11 & 168.4 & 3.08 & $\mathrm{~N}$-atom lone pair \\
\hline HIRWAI & $M(I) \cdots A(N)$ & $C 2 / c$ & 2.12 & 177.8 & 2.99 & $\mathrm{~N}$-atom lone pair \\
\hline HIRWEM & $M(I) \cdots A(N)$ & $P-1$ & 2.11 & 179.1 & 3.12 & $\mathrm{~N}$-atom lone pair \\
\hline HIRWOW & $A(I) \cdots M(N)$ & $P-1$ & 2.11 & 173.3 & 3.34 & $\pi$-system \\
\hline JIKJAR & $\beta \cdots$ Pyrrole & $P-1$ & 2.07 & 153.6 & 3.24 & $\pi$-system \\
\hline JUZQAY & $\beta \cdots$ Pyrrole & $P 2_{1} / \mathrm{c}$ & 2.07 & 142.7 & 3.47 & $\pi$-system \\
\hline JUZQAY & $\beta \cdots$ Pyrrole & $P 2_{1} / \mathrm{c}$ & 2.07 & 139.6 & 3.46 & $\pi$-system \\
\hline JUZQEC & $\beta \cdots$ Pyrrole & $P-1$ & 2.08 & 120.9 & 3.50 & $\pi$-system \\
\hline JUZQIG & $\beta \cdots$ Pyrrole & $P-1$ & 2.09 & 134.7 & 3.43 & $\pi$-system \\
\hline JUZQIG & $\beta \cdots$ Pyrrole & $P-1$ & 2.12 & 135.3 & 3.44 & $\pi$-system \\
\hline JUZQIG & $\beta \cdots$ Pyrrole & $P-1$ & 2.07 & 137.1 & 3.44 & $\pi$-system \\
\hline JUZQIG & $\beta \cdots$ Pyrrole & $P-1$ & 2.04 & 134.1 & 3.43 & $\pi$-system \\
\hline LOHVOV & $M(I) \cdots A(N)$ & $P 1$ & 2.09 & 176.4 & 3.22 & $\pi$-system \\
\hline SODBET & $M \cdots M$ & $P 1$ & 2.12 & 176.7 & 2.92 & $\mathrm{~N}$-atom lone pair \\
\hline SODBET & $M \cdots M$ & $P 1$ & 2.09 & 178.7 & 3.44 & $\pi$-system \\
\hline SODBUJ & $\mathrm{M} \cdots \mathrm{M}$ & $P-1$ & 2.11 & 162.6 & 3.32 & $\pi$-system \\
\hline SODBUJ & $\mathrm{M} \cdots \mathrm{M}$ & $P-1$ & 2.11 & 170.7 & 3.39 & $\pi$-system \\
\hline VAPVAK & $\beta \cdots$ Pyrrole & Pcab & 2.02 & 167.1 & 3.22 & $\pi$-system \\
\hline VONGAI & $M(I) \cdots A(N)$ & $C 2 / \mathrm{c}$ & 2.12 & 165.9 & 3.47 & $\pi$-system \\
\hline VONGIQ & $M(I) \cdots A(N)$ & $P-1$ & 2.10 & 164.6 & 3.13 & $\mathrm{~N}$-atom lone pair \\
\hline VONGIQ & $M(I) \cdots A(N)$ & $P-1$ & 2.12 & 173.3 & 3.00 & $\mathrm{~N}$-atom lone pair \\
\hline VONHEN & $A(I) \cdots M(N)$ & $P 2_{1} / \mathrm{n}$ & 2.10 & 156.4 & 3.31 & $\mathrm{~N}$-atom lone pair \\
\hline
\end{tabular}




\section{Crystal Structures}

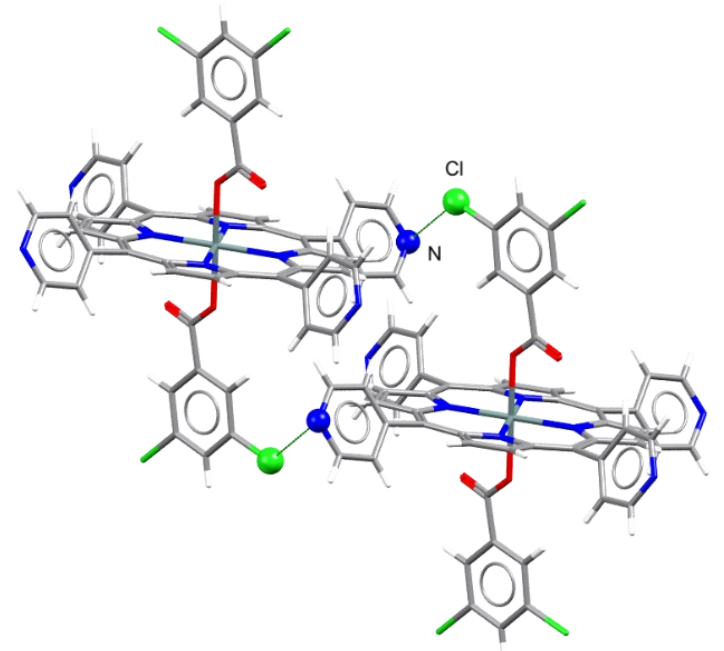

Figure S12. View of the crystal structure with the CSD code CERWEE.

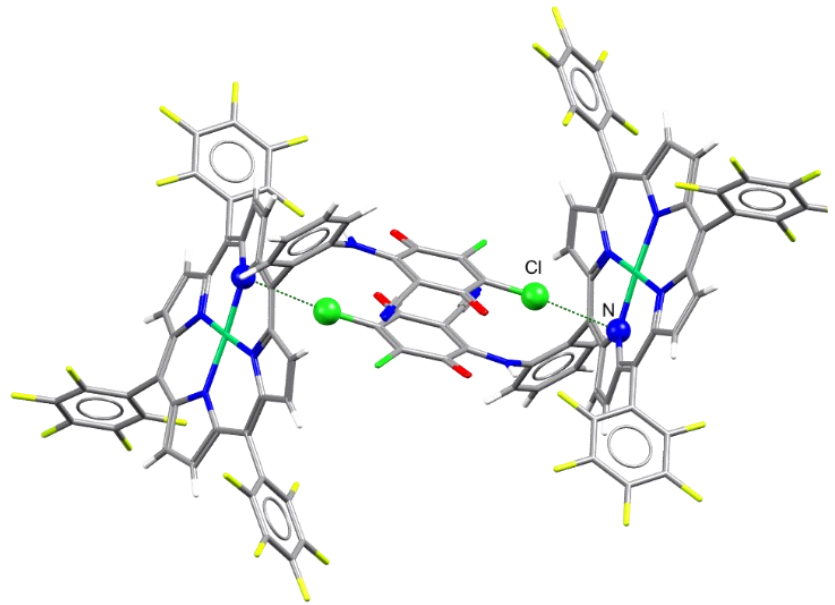

Figure S13. View of the crystal structure with the CSD code MULVUK, with solvents and disordered moieties omitted for clarity.

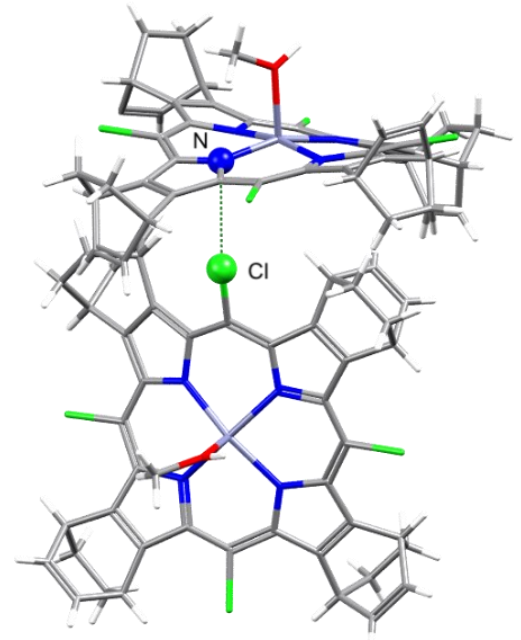

Figure S14. View of the crystal structure with the CSD code QUCZUK, with solvent molecules and disordered units omitted for clarity. 


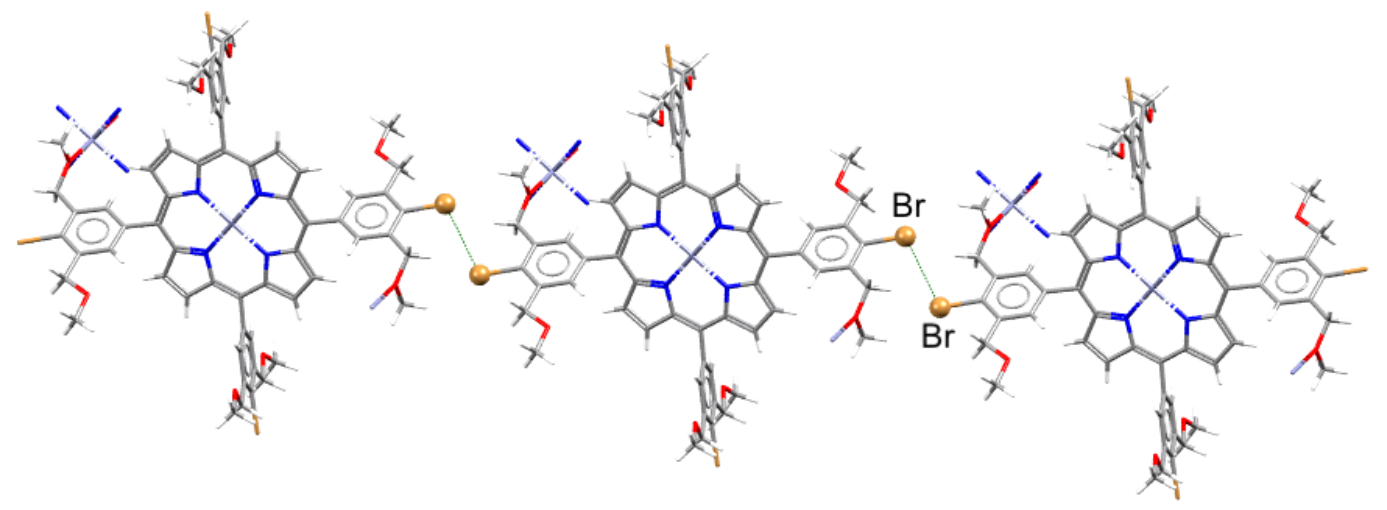

Figure S15. View of the crystal structure with the CSD code NIFSIF.

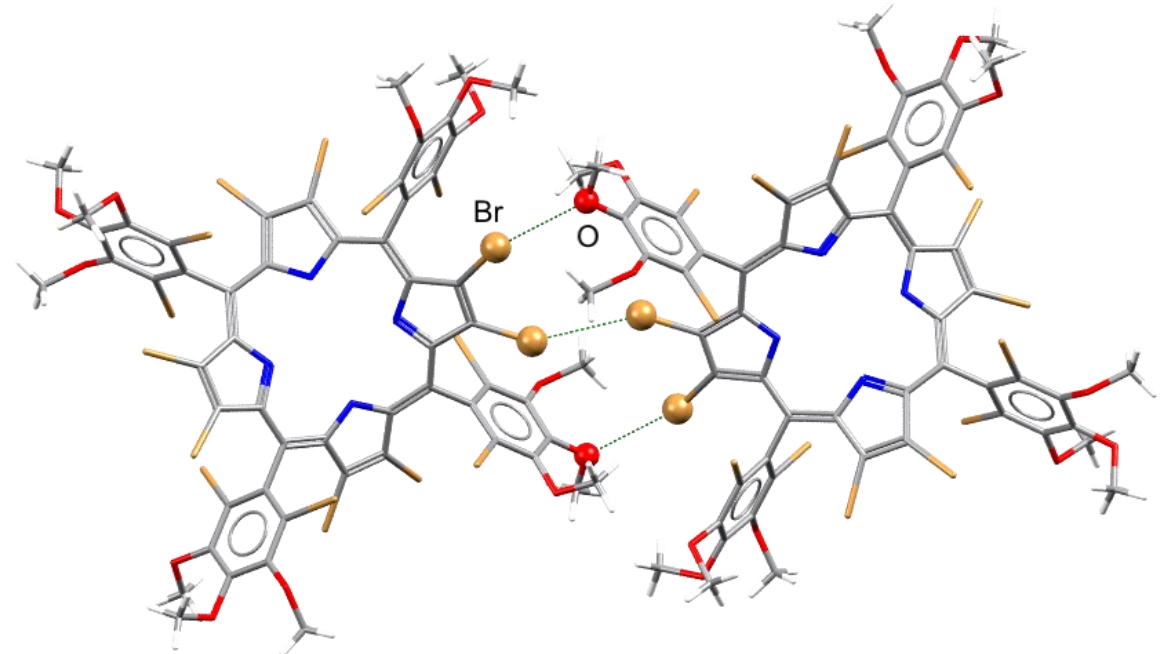

Figure S16. View of the crystal structure with the CSD code BAKHON.

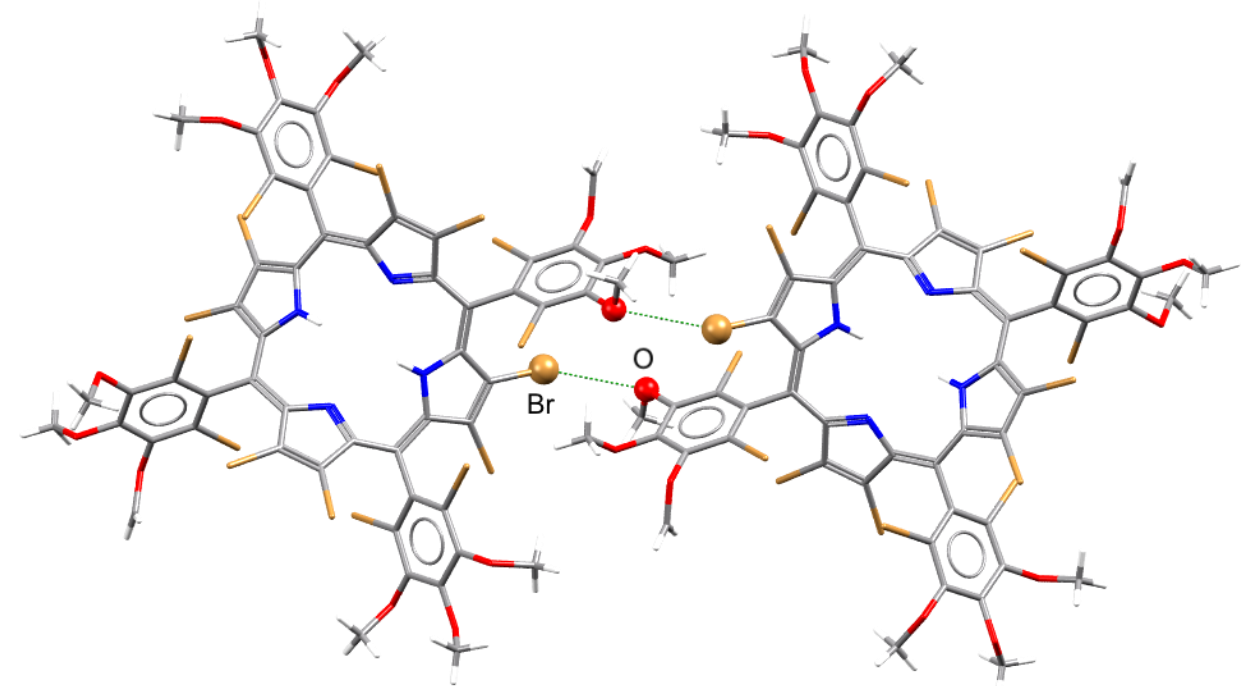

Figure S17. View of the crystal structure with the CSD code BAKJAB, with solvent molecules and disordered moieties omitted for clarity. 


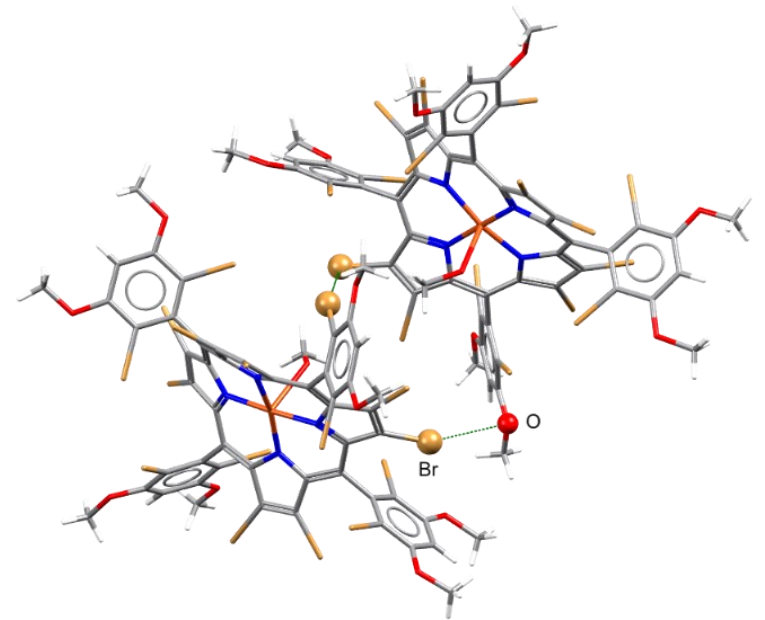

Figure S18. View of the crystal structure with the CSD code FEYMUS, with solvent molecules and disordered moieties omitted for clarity.

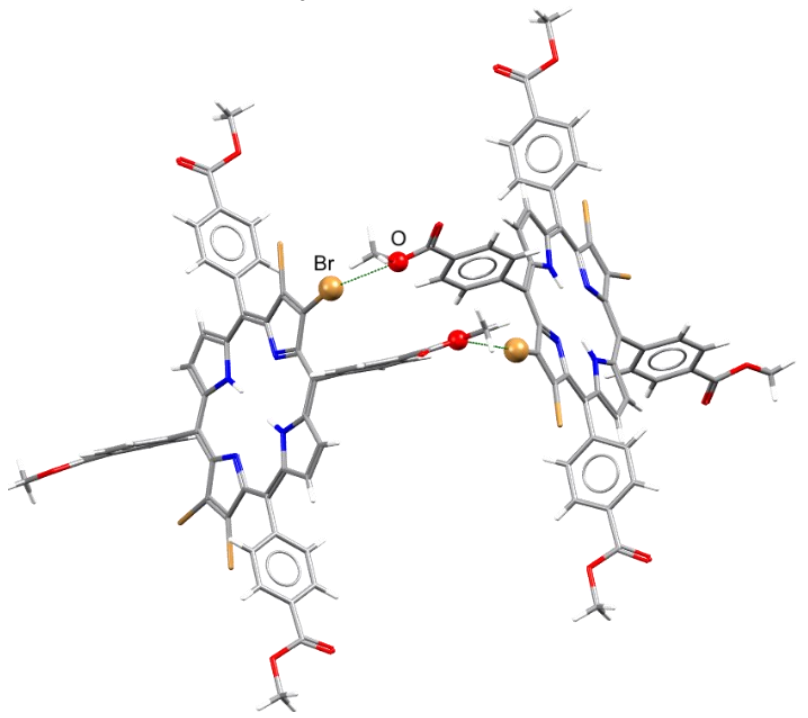

Figure S19. View of the crystal structure with the CSD code UFOFOL, with solvent molecules and disordered moieties omitted for clarity.

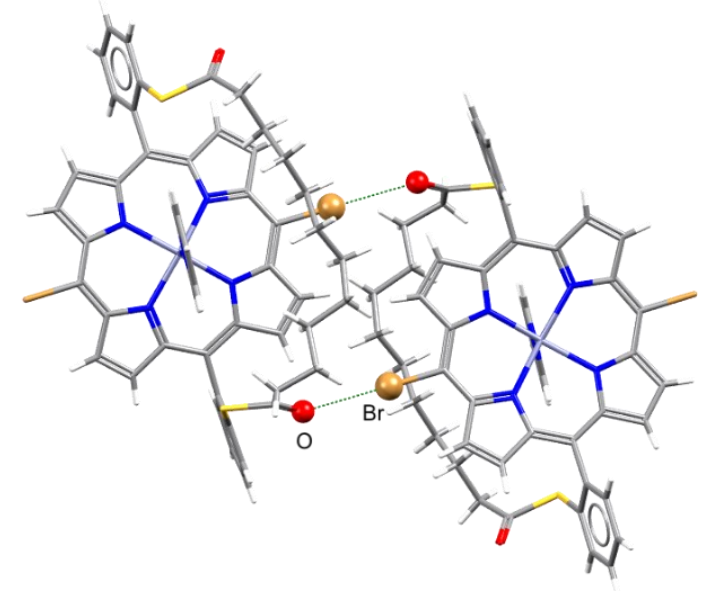

Figure S20. View of the crystal structure with the CSD code KAXWIS, with disordered moieties omitted for clarity. 


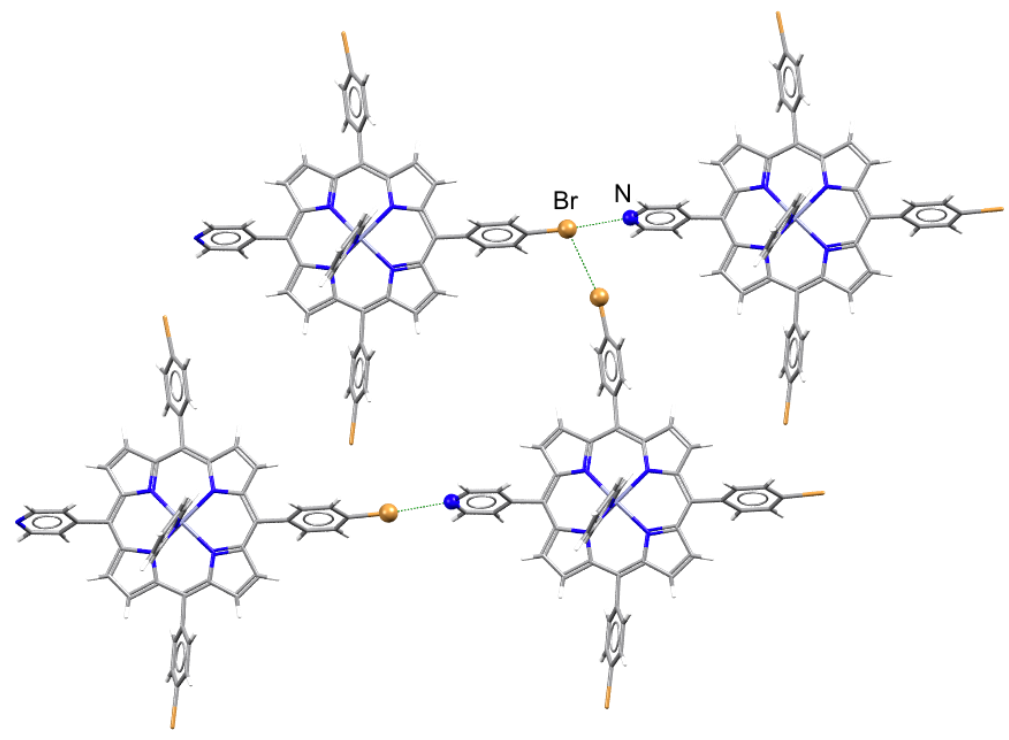

Figure S21. View of the crystal structure with CSD code DEGREO, with solvent molecules omitted for clarity.

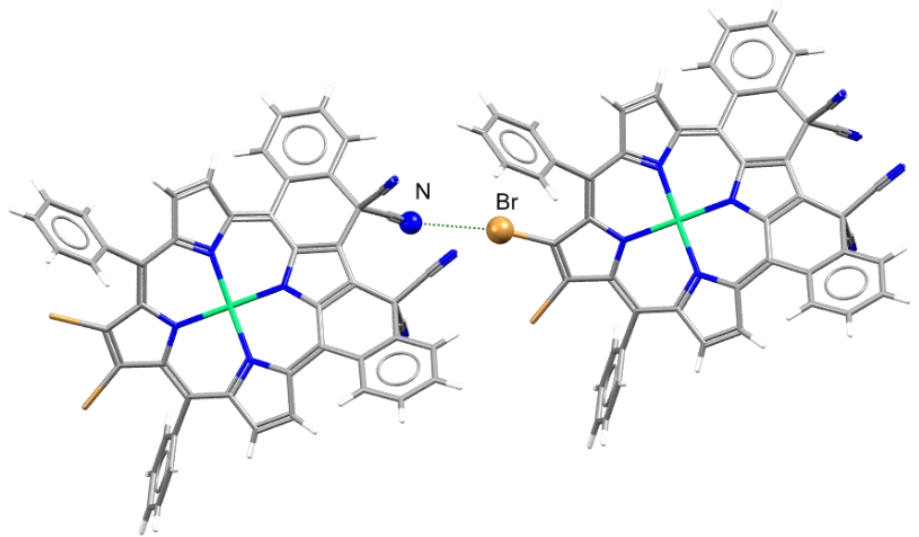

Figure S22. View of the crystal structure with CSD code DAQWEA, solvent molecules omitted for clarity.

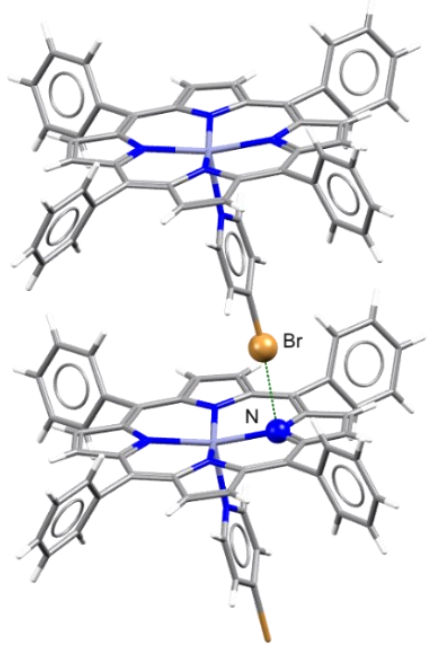

Figure S23. View of the crystal structure with the CSD code NANZIO, with solvent molecules omitted for clarity. 


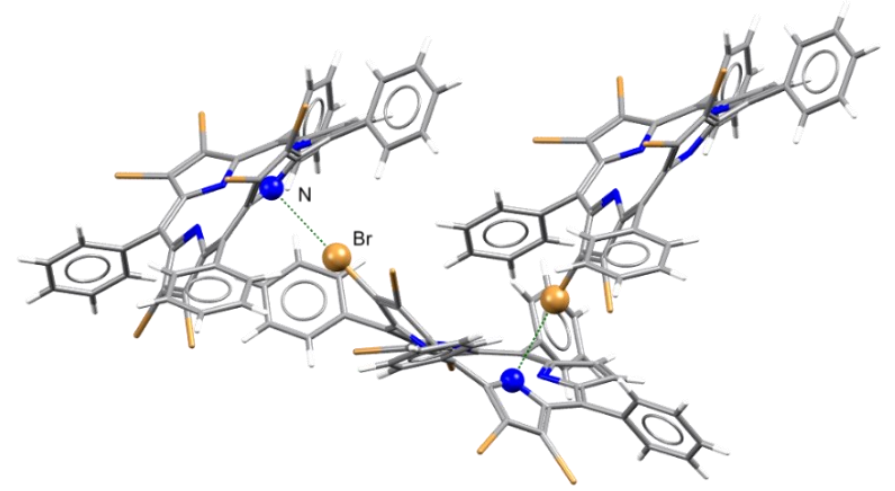

Figure S24. View of the crystal structure with the CSD code LEVQEI, with solvent molecules omitted for clarity.
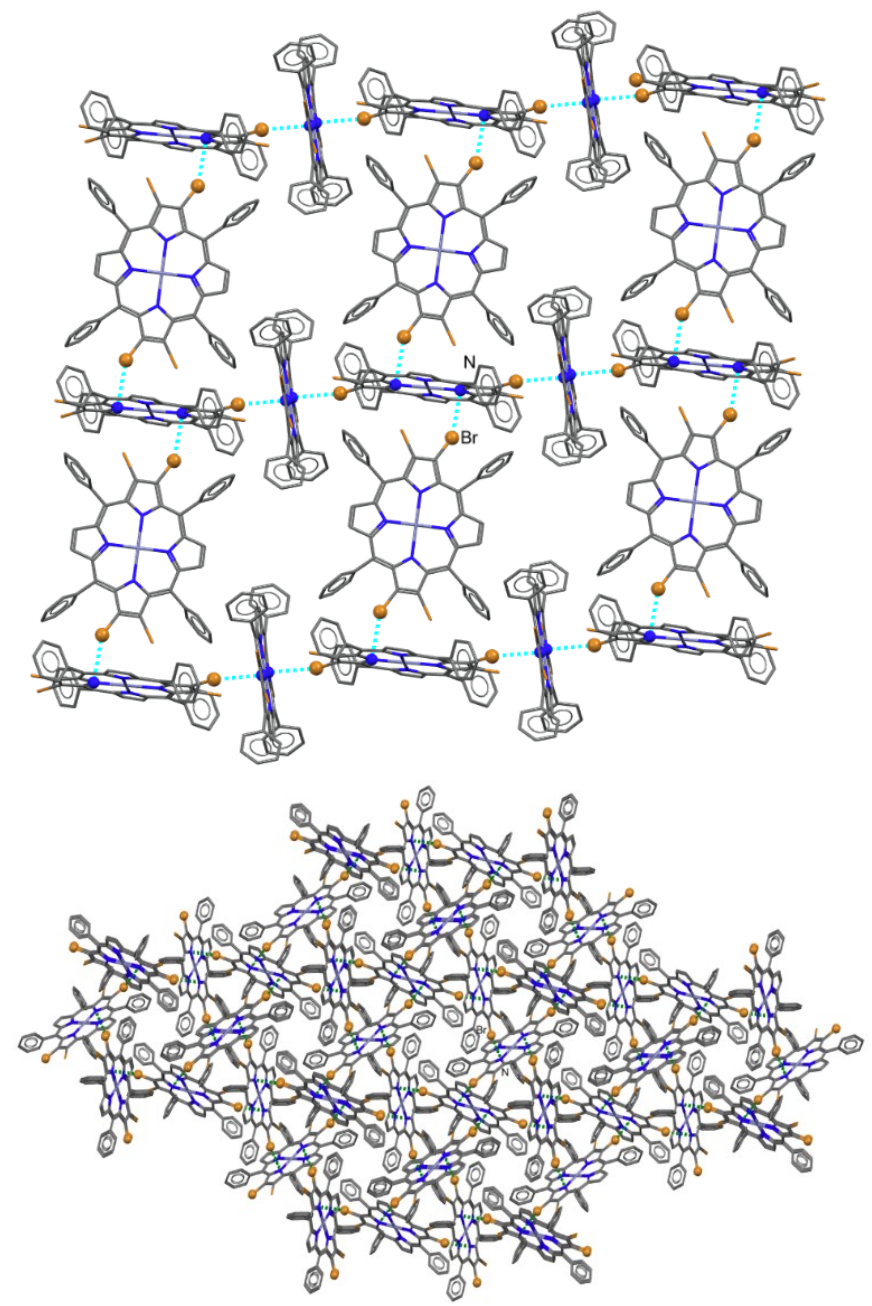

Figure S25. Different views of the crystal structure with the CSD code RECMUH: (top) parallel to the crystallographic 110-plane and (bottom) along the crystallographic c-axis. The structure deposited in the CSD does not contain any hydrogen atoms, and solvent molecules are omitted for clarity. 


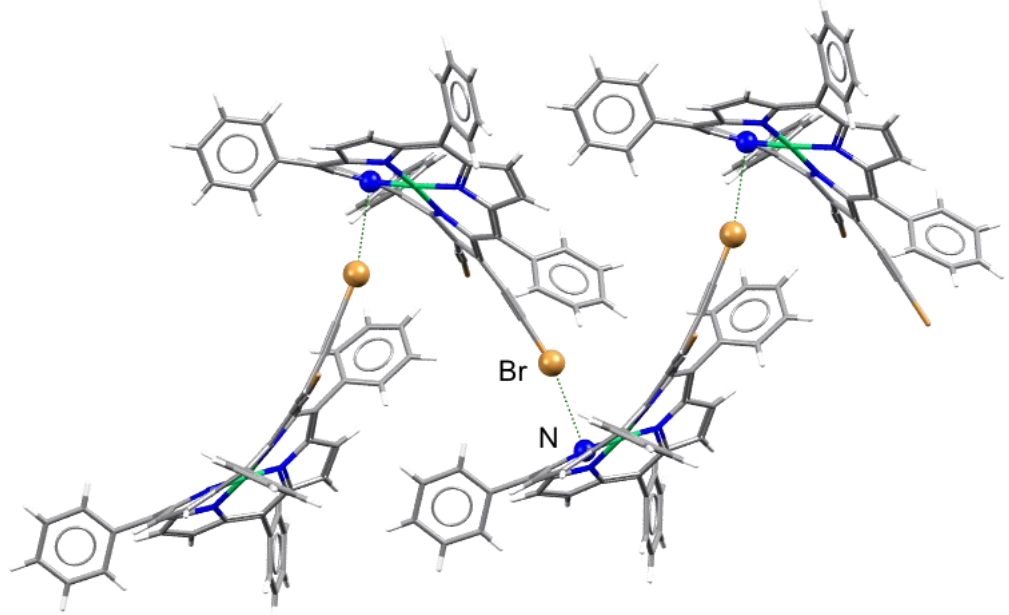

Figure S26. View of the crystal structure with the CSD code VAPVEO, with solvent molecules and disordered moieties omitted for clarity.

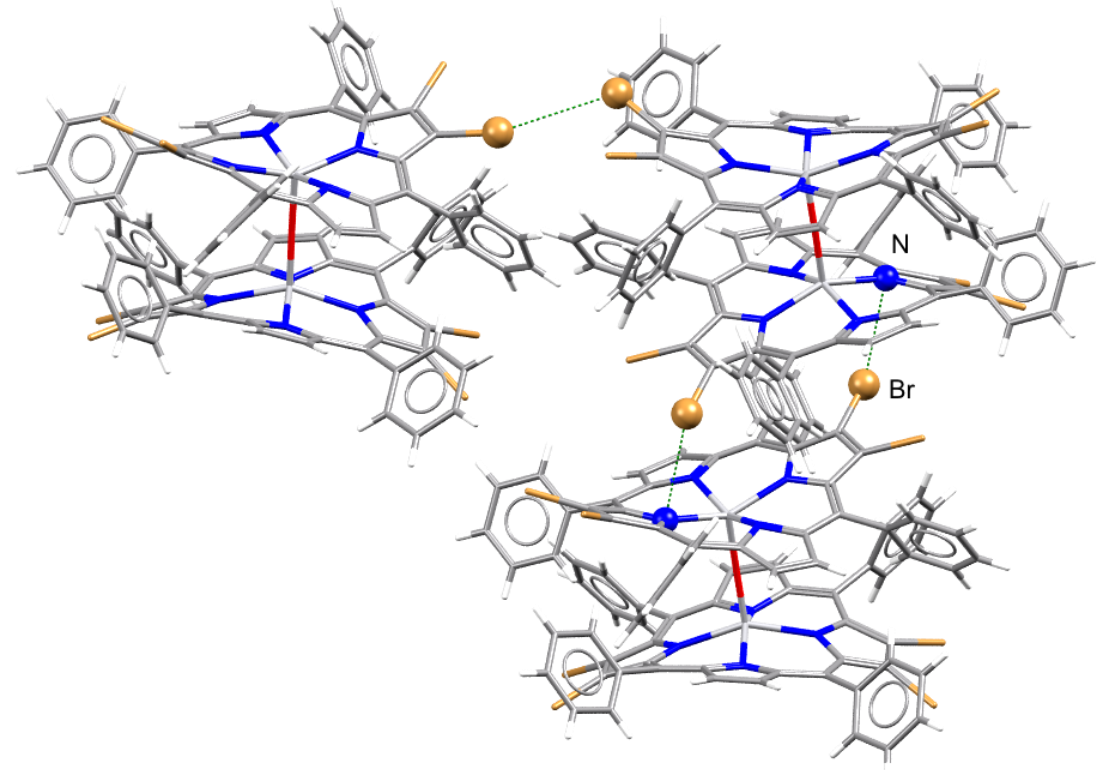

Figure S27. View of the crystal structure with the CSD code COGWED.

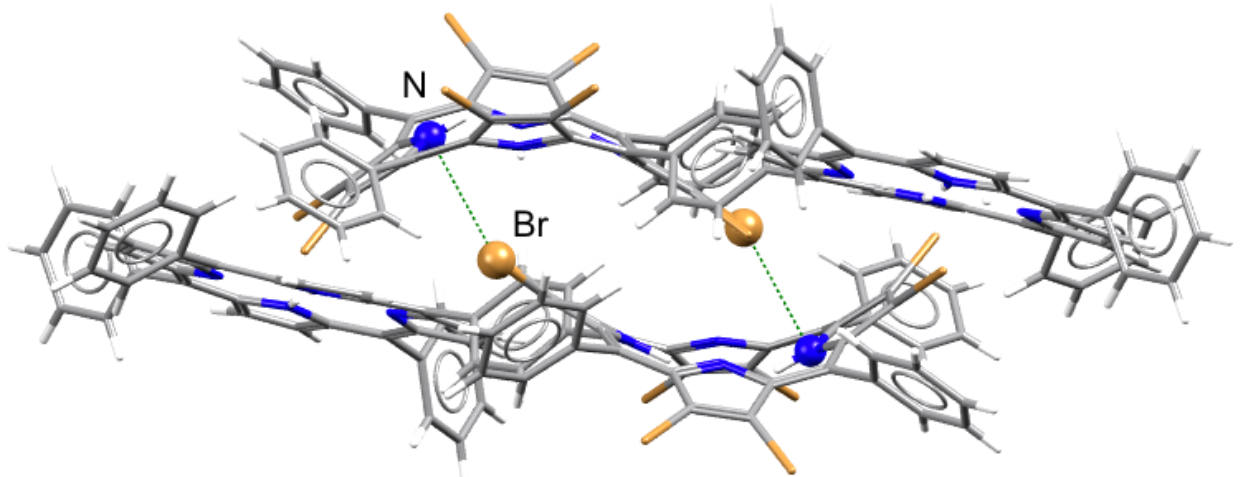

Figure S28. View of the crystal structure with the CSD code FASLER, with solvent molecules omitted for clairity. 


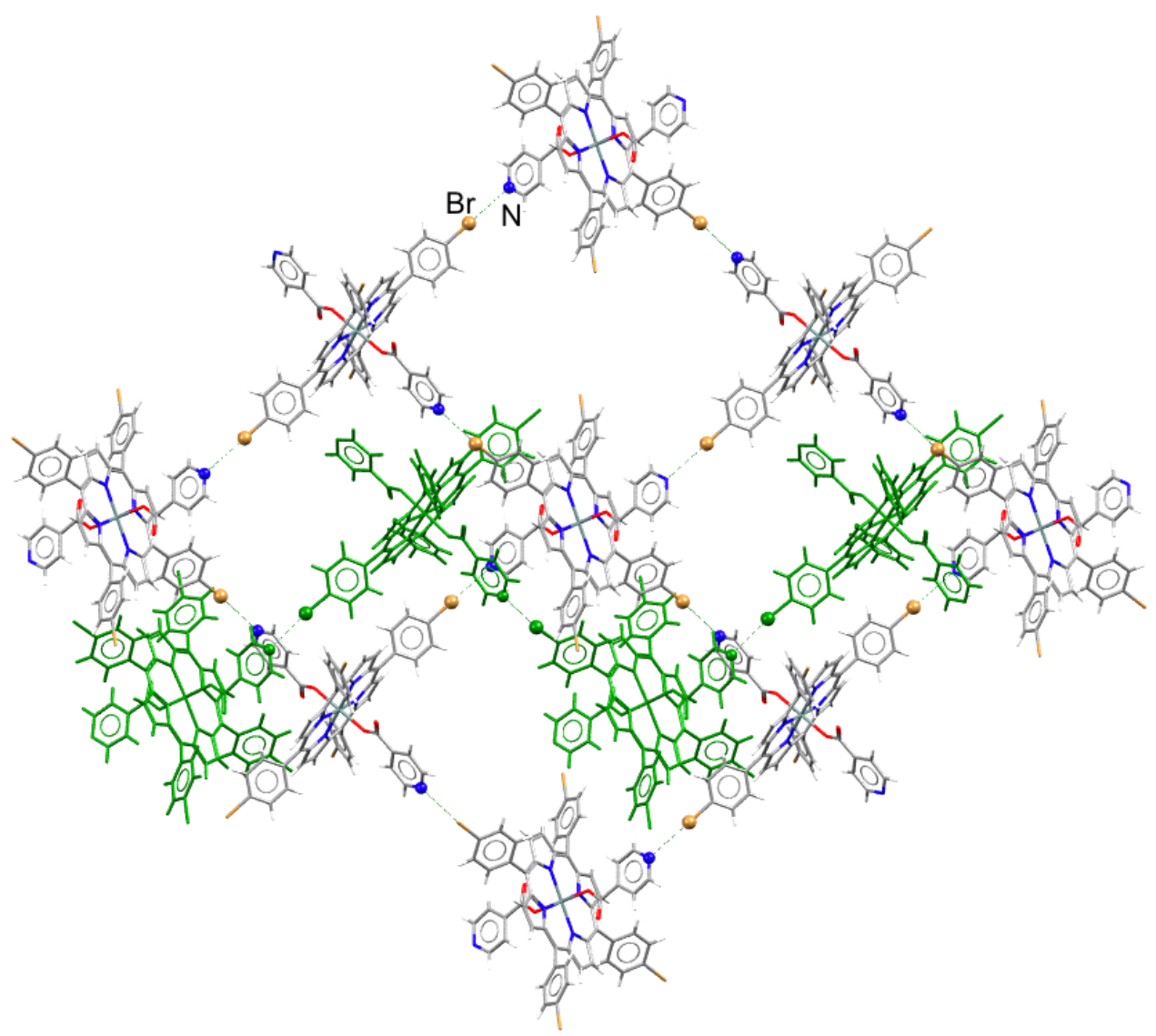

Figure S29. View of the crystal structure with the CSD code HIRWIQ.

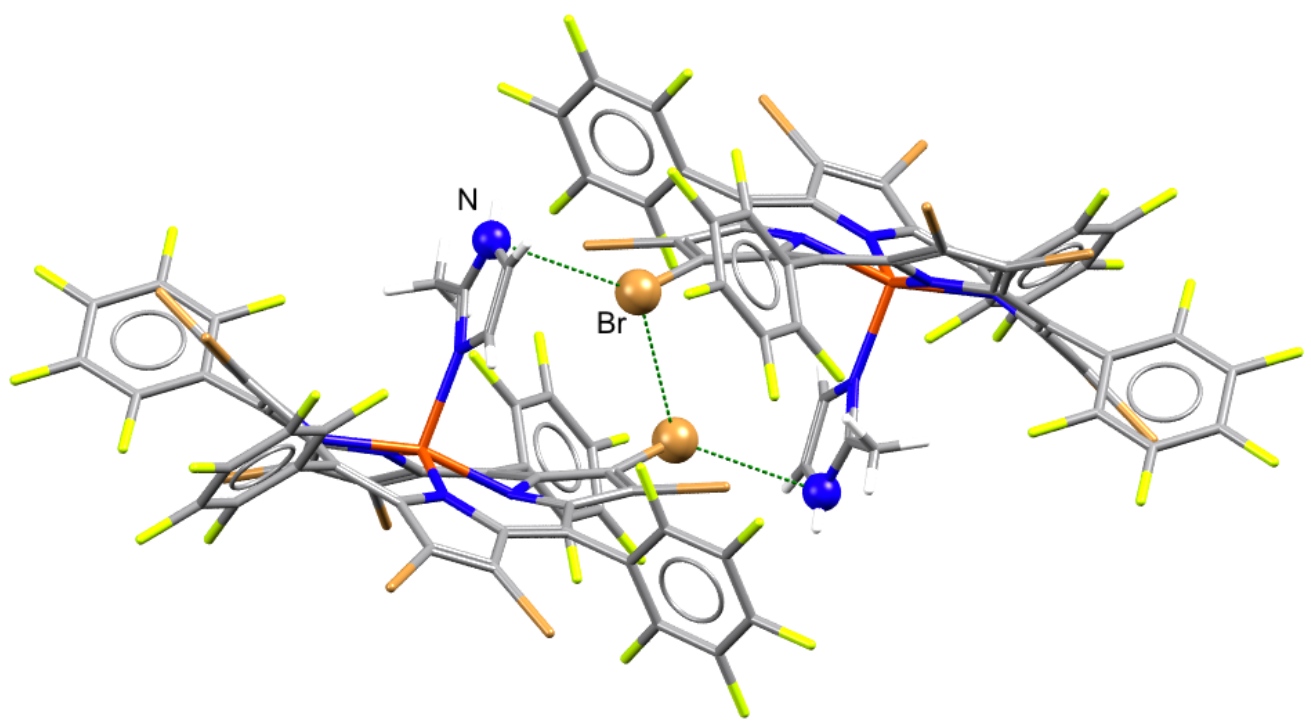

Figure S30. View of the crystal structure with the CSD code EWUROF, with disordered moieties omitted for clarity. 


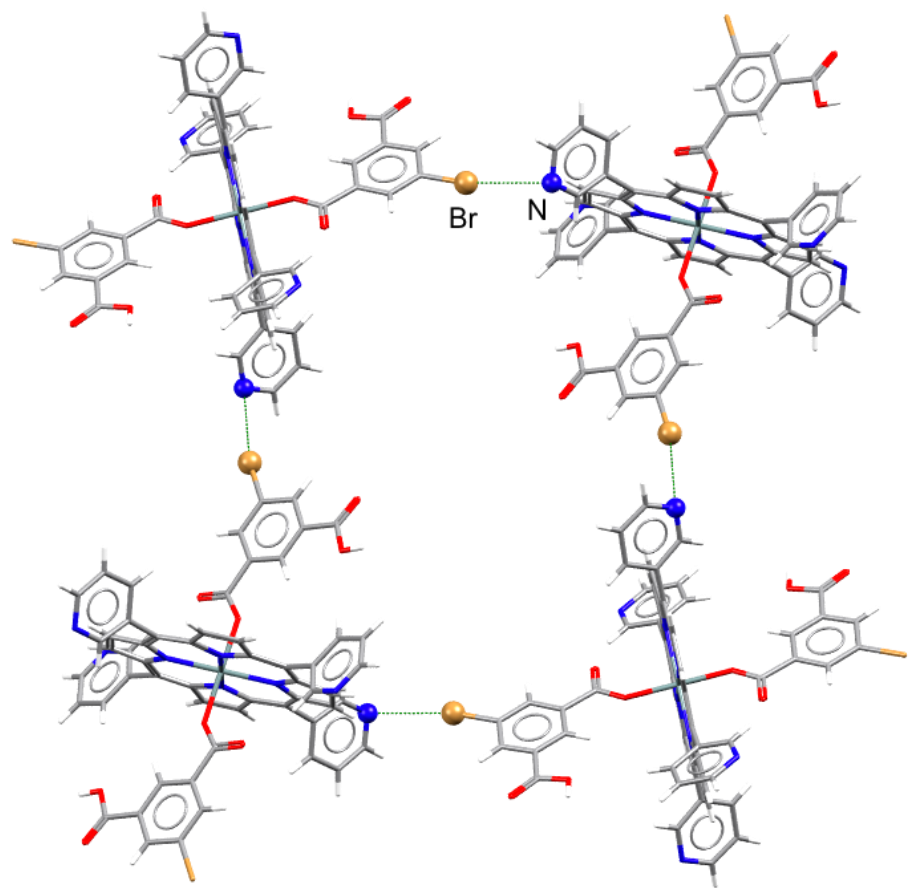

Figure S31. View of the crystal structure with the CSD code XICTUA.

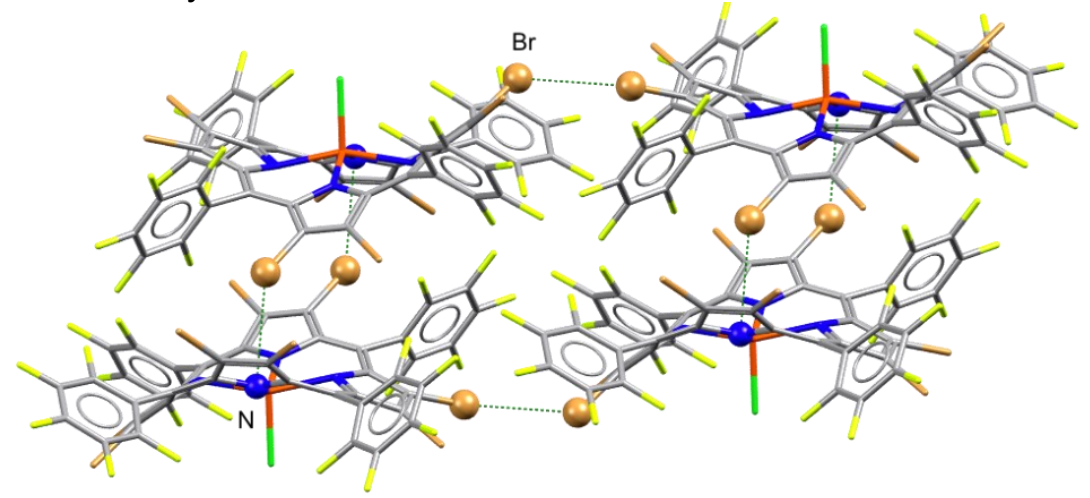

Figure S32. View of the crystal structure with the CSD code ZEQQUH.

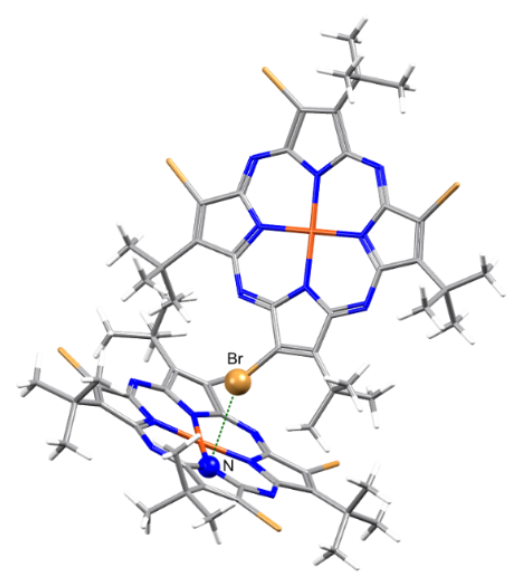

Figure S33. View of the crystal structure with the CSD code WAHBEP. 


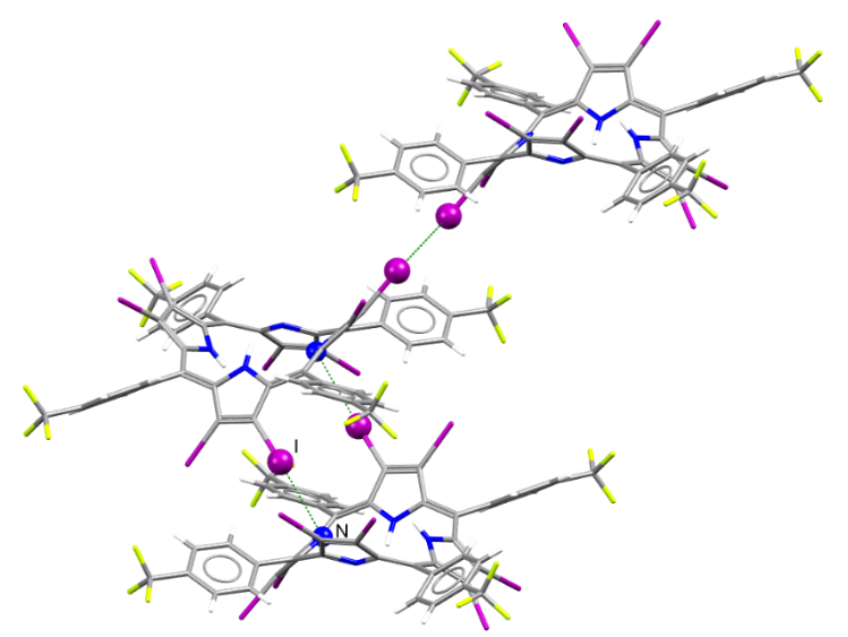

Figure S34. View of the crystal structure with the CSD code JIKJAR, with solvent molecules and disordered moieties omitted for clarity.

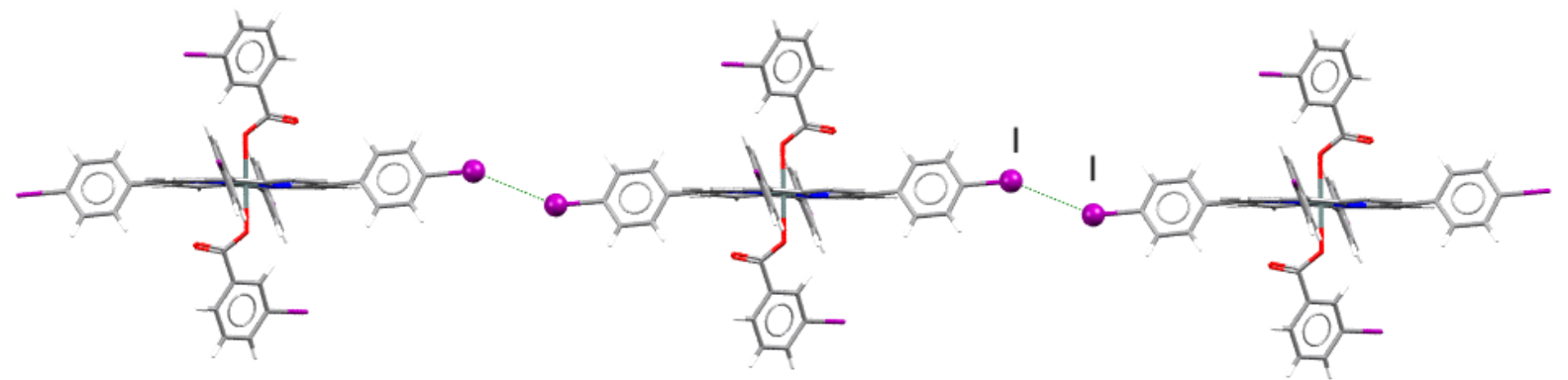

Figure S35. View of the crystal structure with the CSD code KIWTOC.

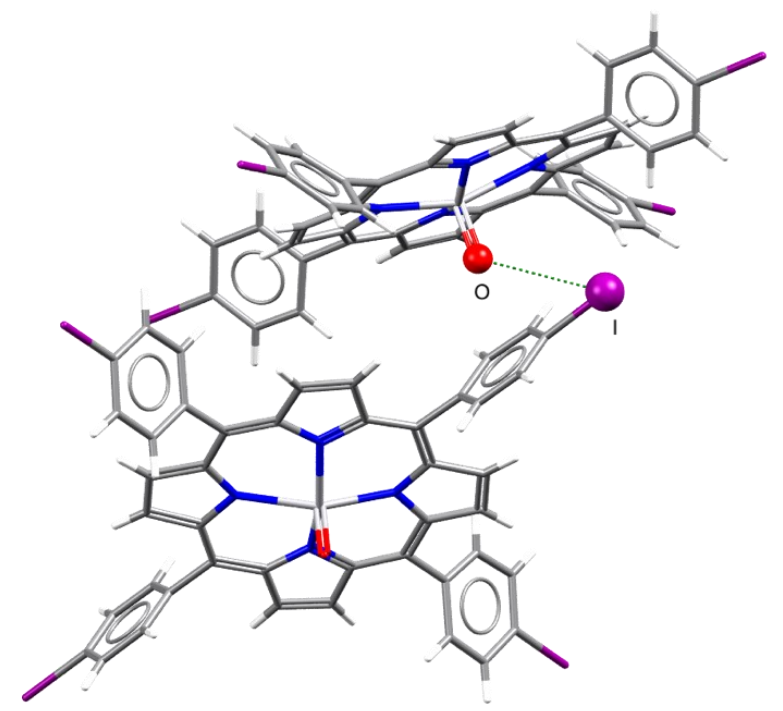

Figure S36. View of the crystal structure with the CSD code VONFUB. This entry was not included in our analysis due to the unusual geometry of the contact between iodine and oxygen atoms. 


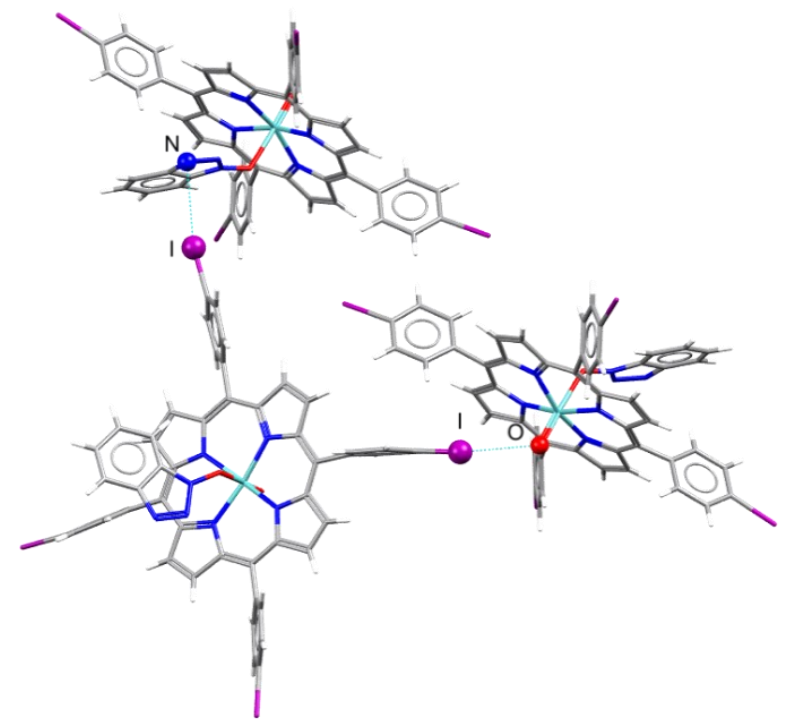

Figure S37. View of the crystal structure with the CSD code VONGAI, with solvent molecules and disordered moieties omitted for clarity.

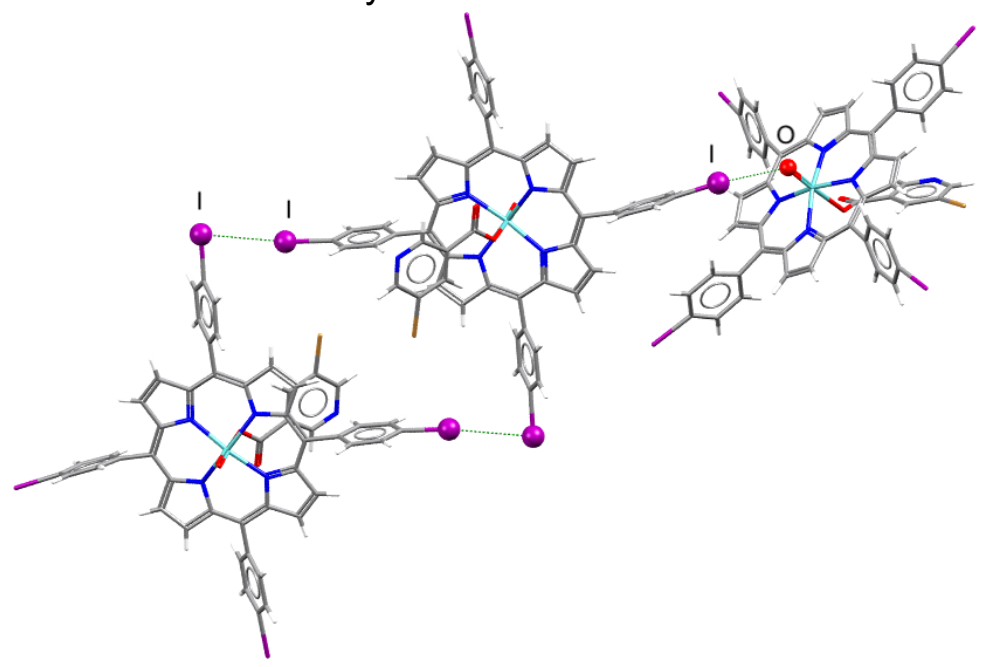

Figure S38. View of the crystal structure with the CSD code VONGEM, with disordered moieties omitted for clarity.

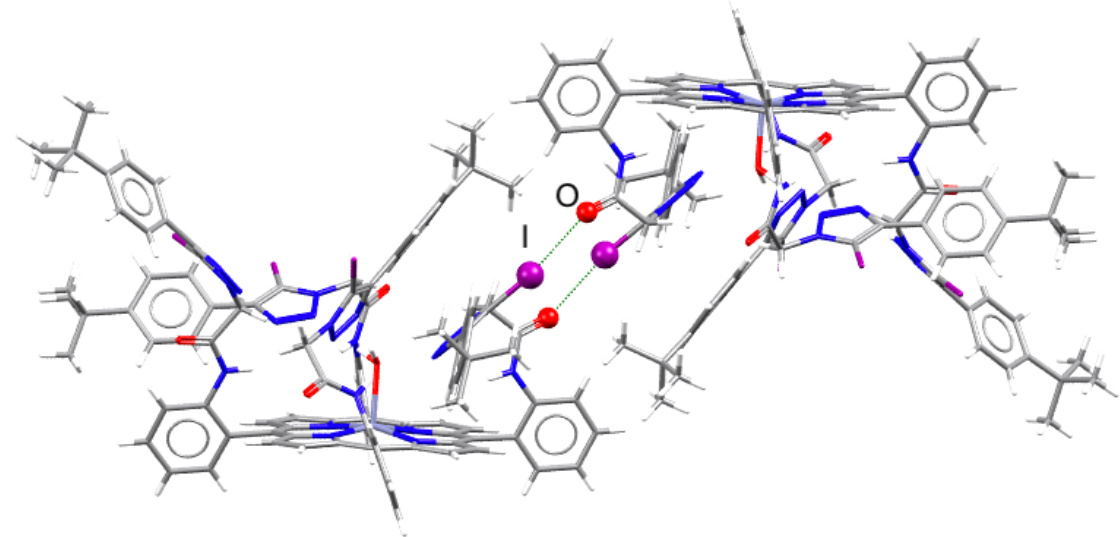

Figure S39. View of the crystal structure with the CSD code AGOKOY, with solvent molecules omitted for clarity. 


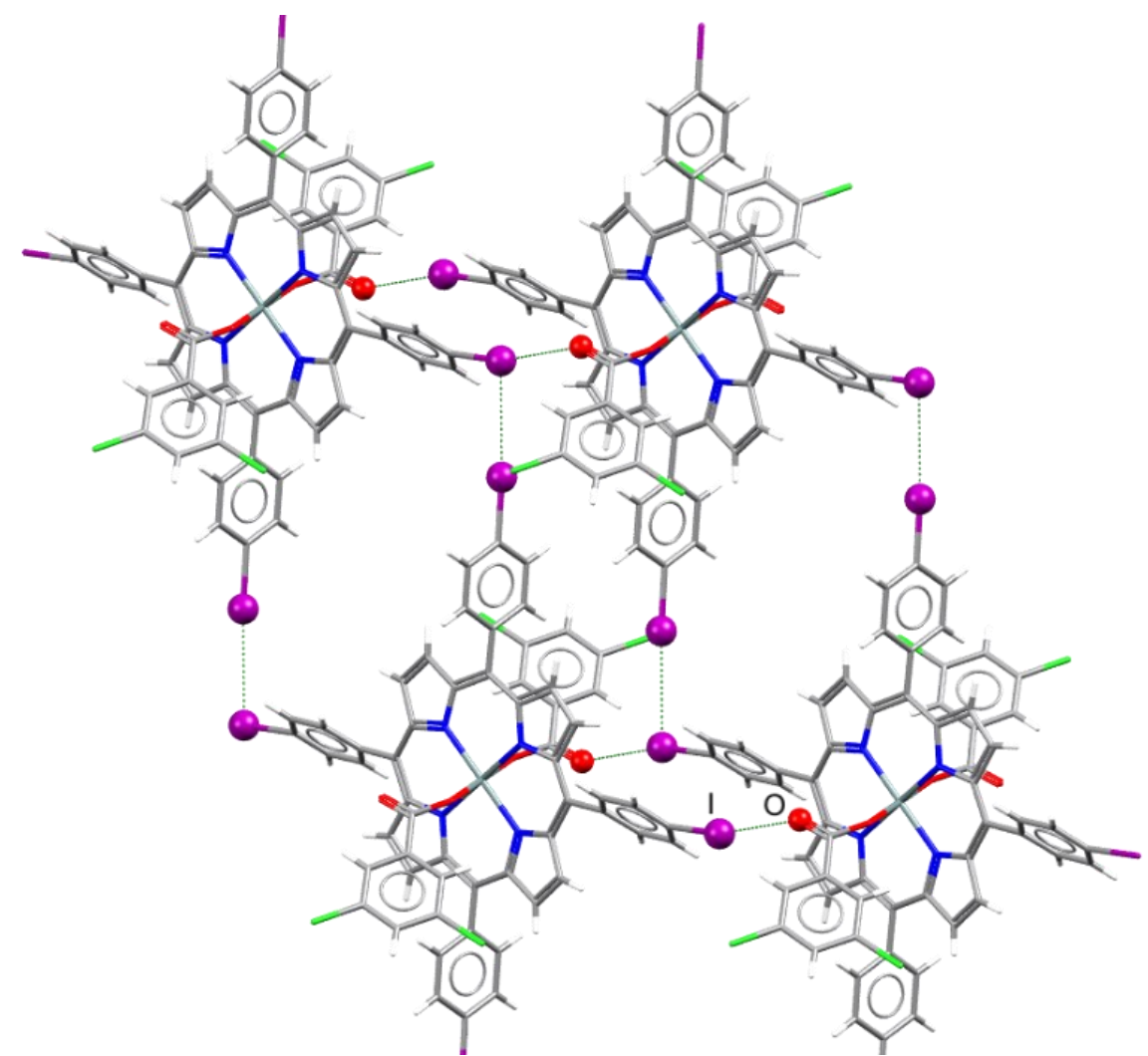

Figure S40. View of the crystal structure with the CSD code KIWVEU.

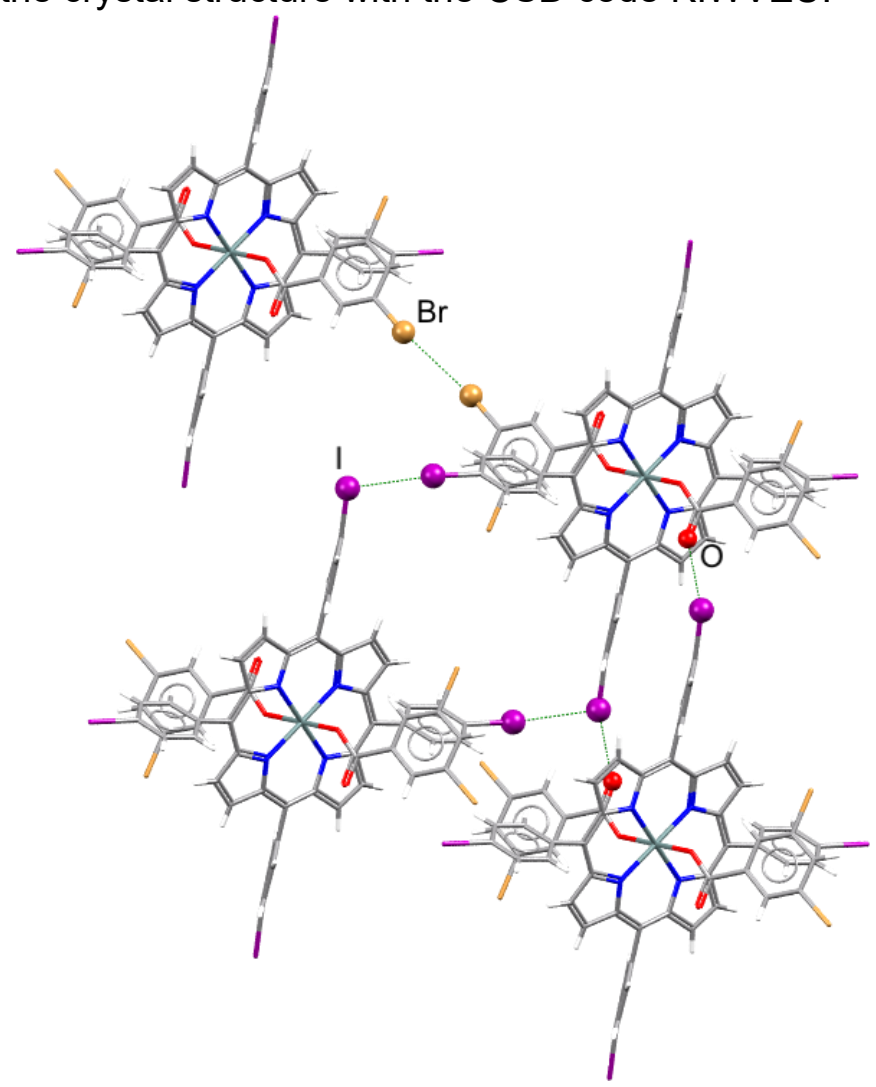

Figure S41. View of the crystal structure with the CSD code KIWVIY. 


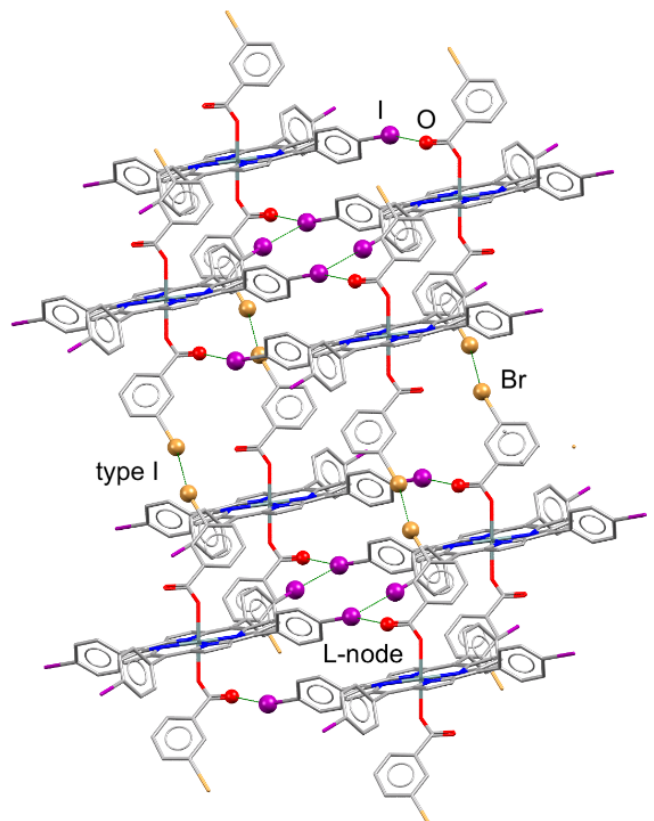

Figure S42. View of the crystal structure with the CSD code ZOZZIA, with disordered moieties omitted for clarity.

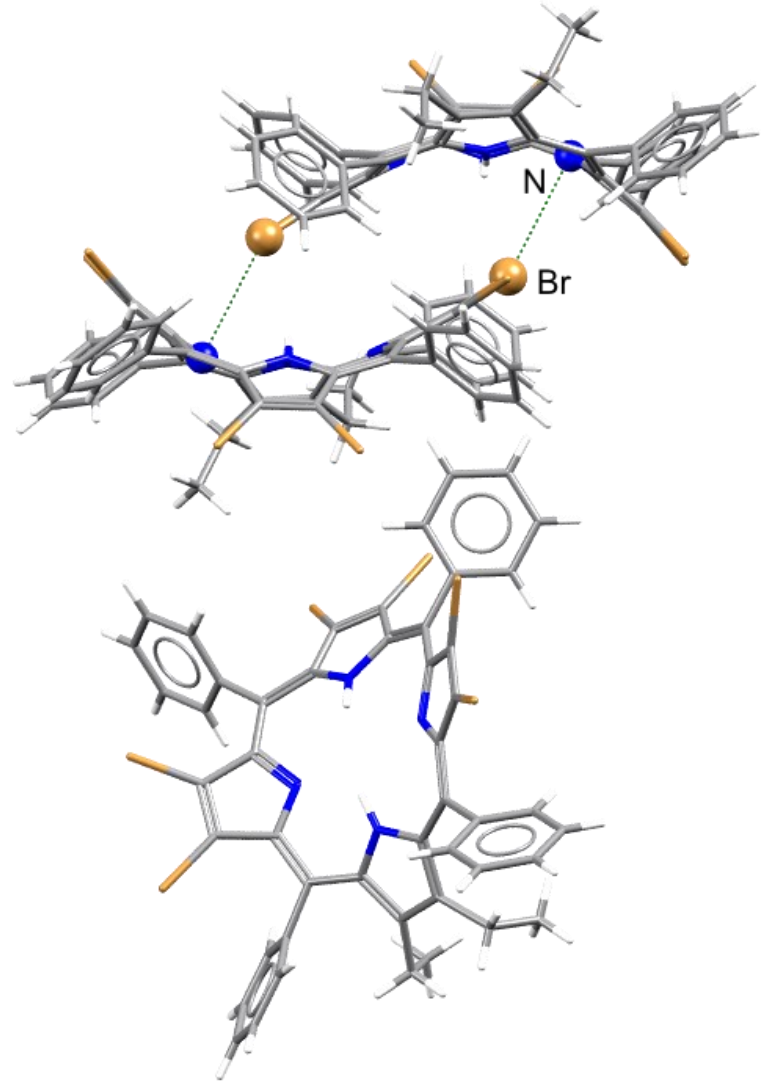

Figure S43. View of the crystal structure with the CSD code GOBWEZ, with solvent molecules and disordered moieties omitted for clarity. 


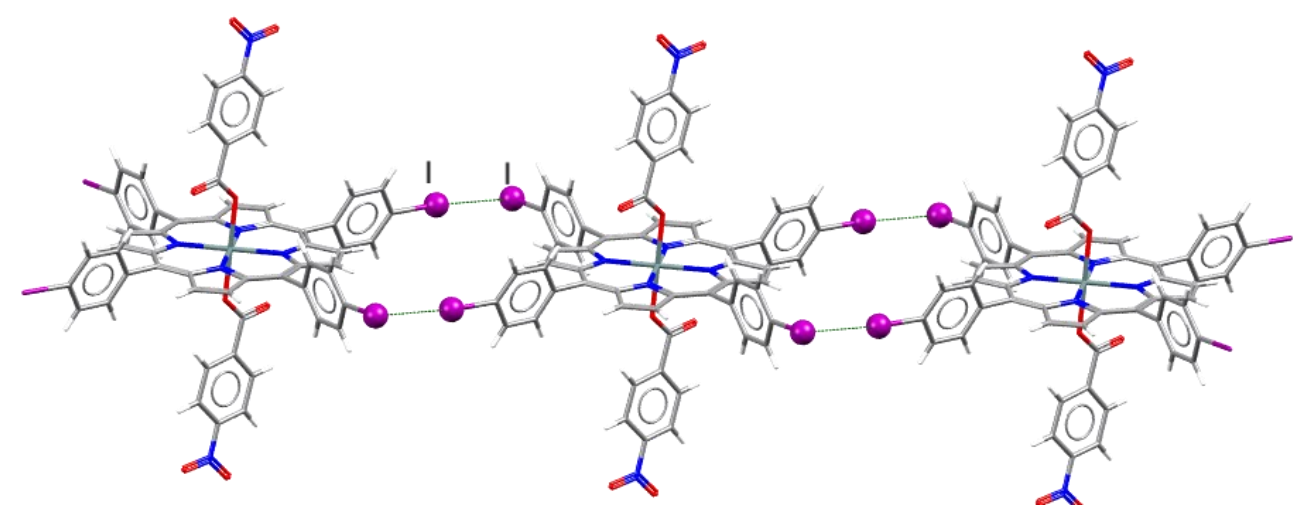

Figure S44. View of the crystal structure with the CSD code KIWVUK, with solvent molecules and disordered moieties omitted for clarity.

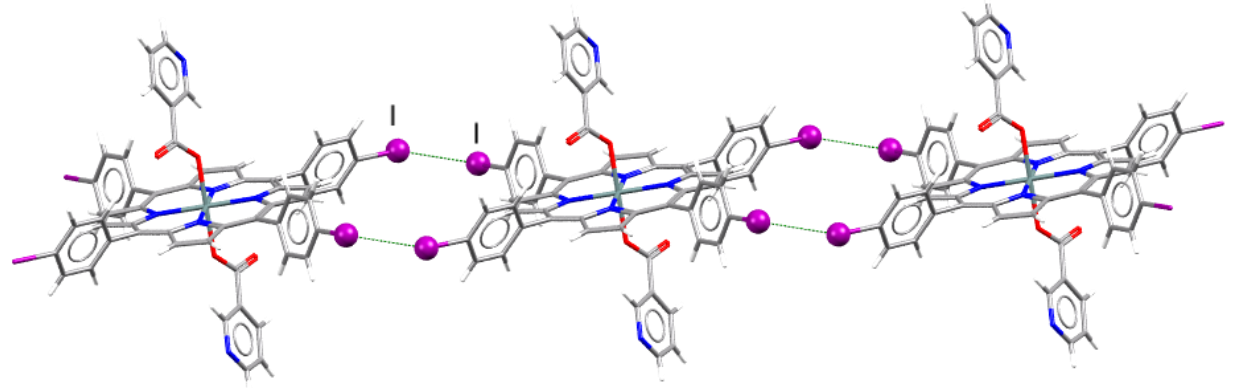

Figure S45. View of the crystal structure with the CSD code VIKREO.

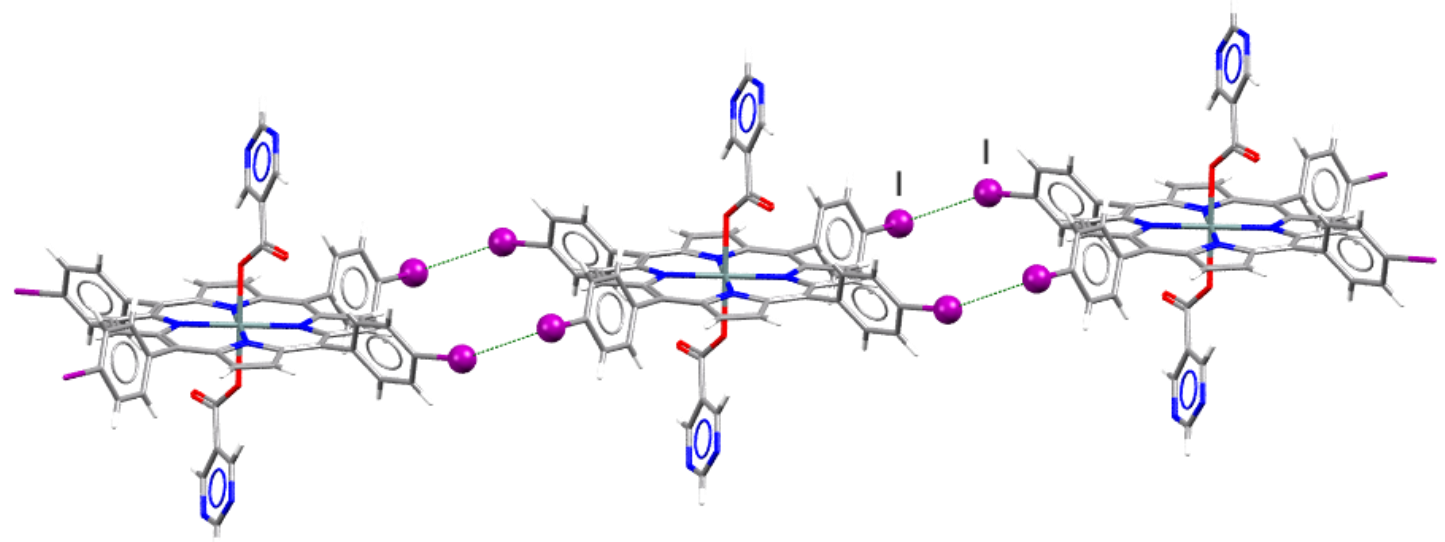

Figure S46. View of the crystal structure with the CSD code VIKRIS.

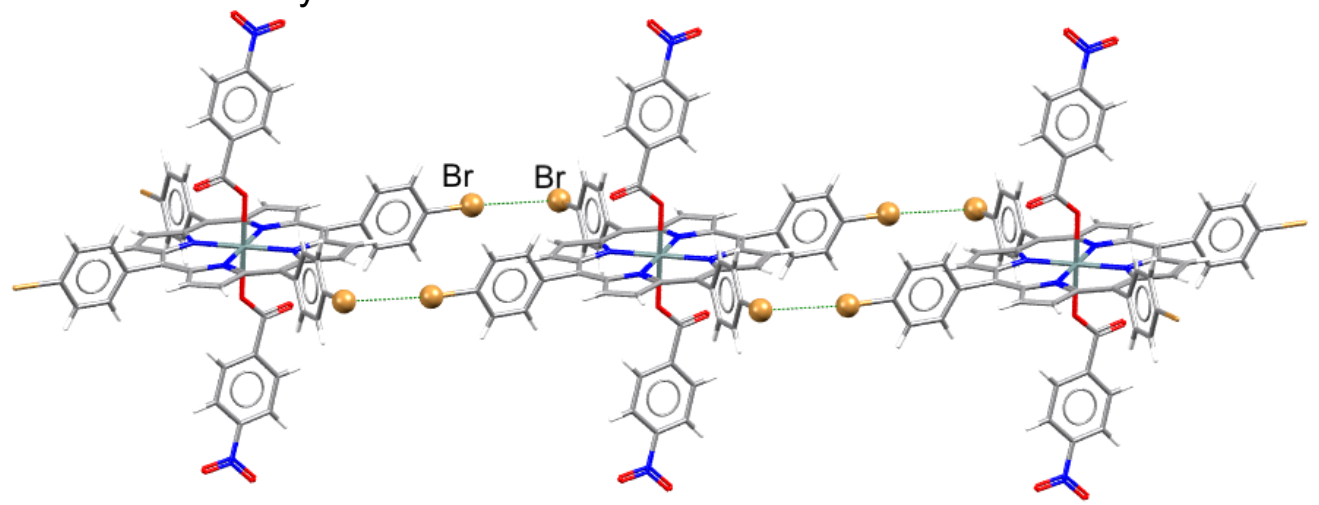

Figure S47. View of the crystal structure with the CSD code XIZLEA, with solvent molecules omitted for clarity. 


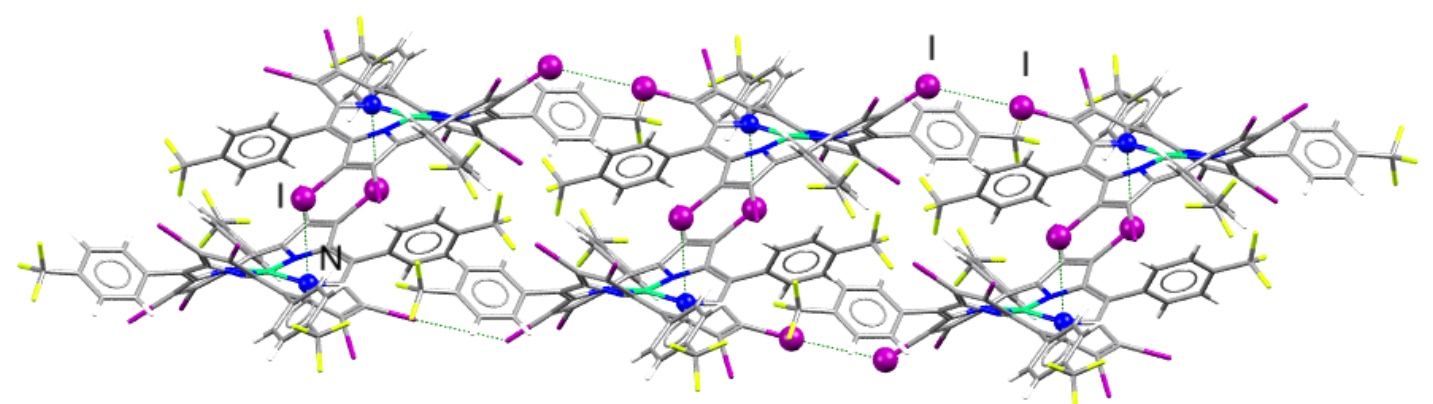

Figure S48. View of the crystal structure with the CSD code JUZQEC, with solvent molecules and disordered moieties omitted for clarity.

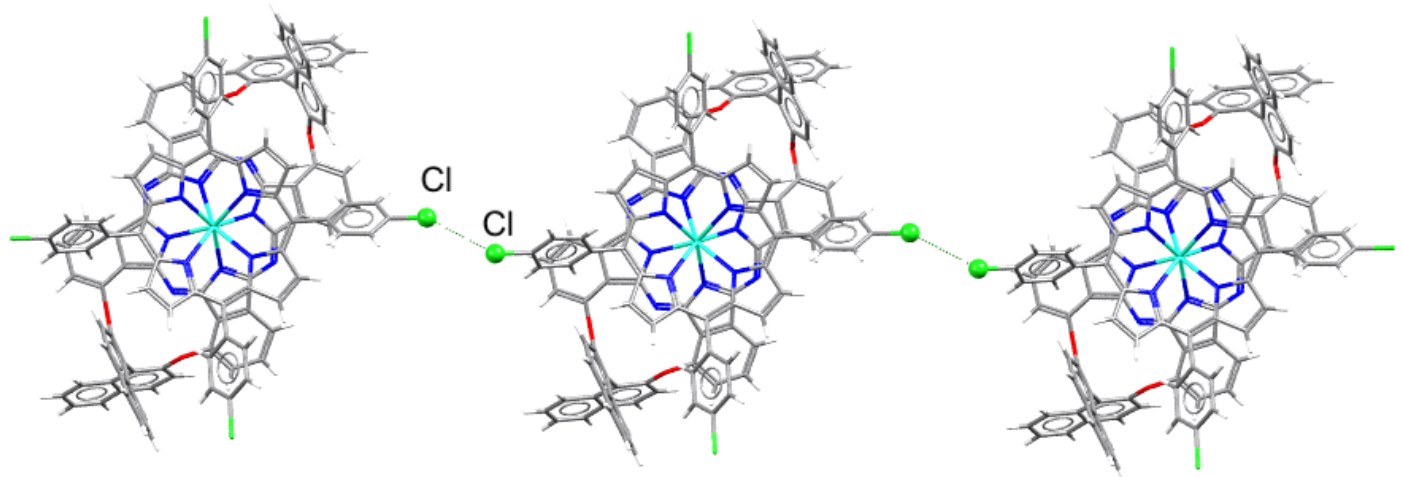

Figure S49. View of the crystal structure with the CSD code SIWKIV, with solvent molecules omitted for clarity.

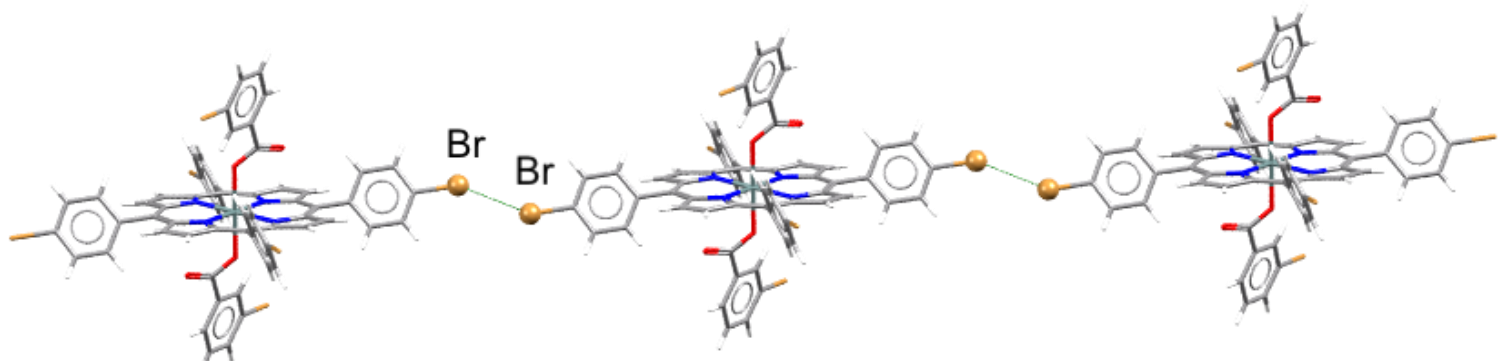

Figure S50. View of the crystal structure with the CSD code XIZKOJ.

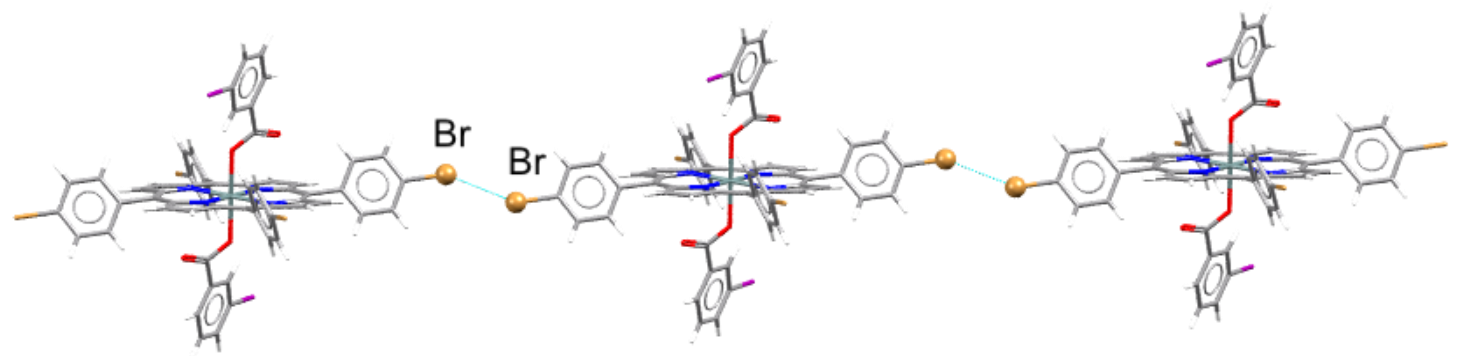

Figure S51. View of the crystal structure with the CSD code XIZKUP. 


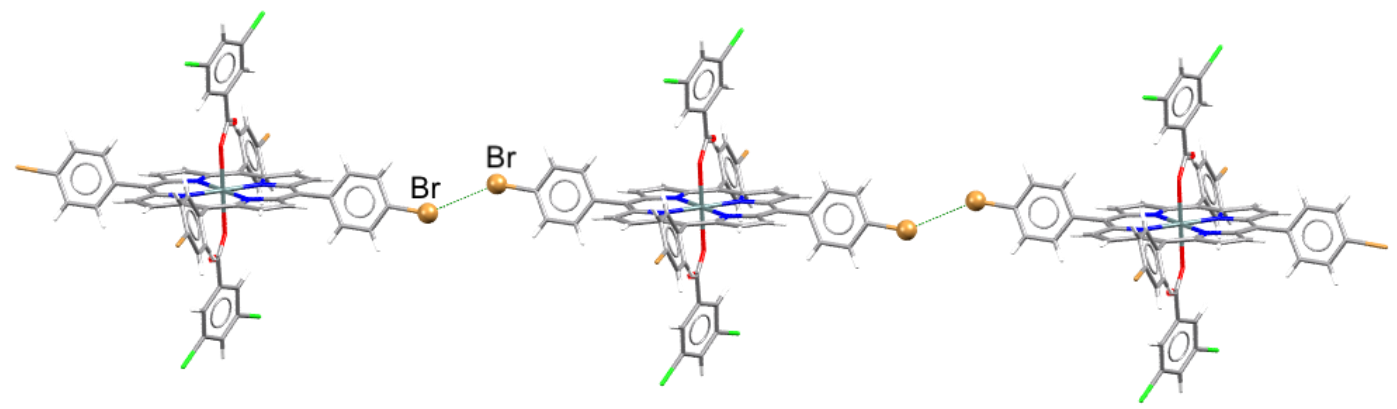

Figure S52. View of the crystal structure with the CSD code XIZLUQ, with solvent molecules omitted for clarity.

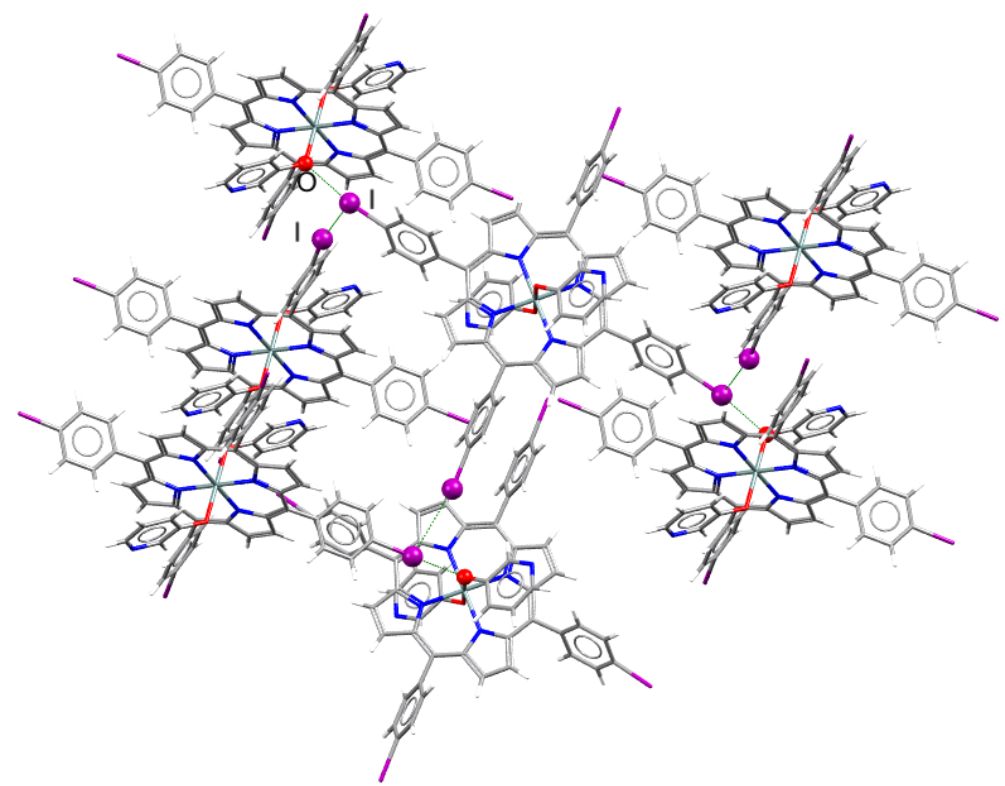

Figure S53. View of the crystal structure with the CSD code LUGMOR.

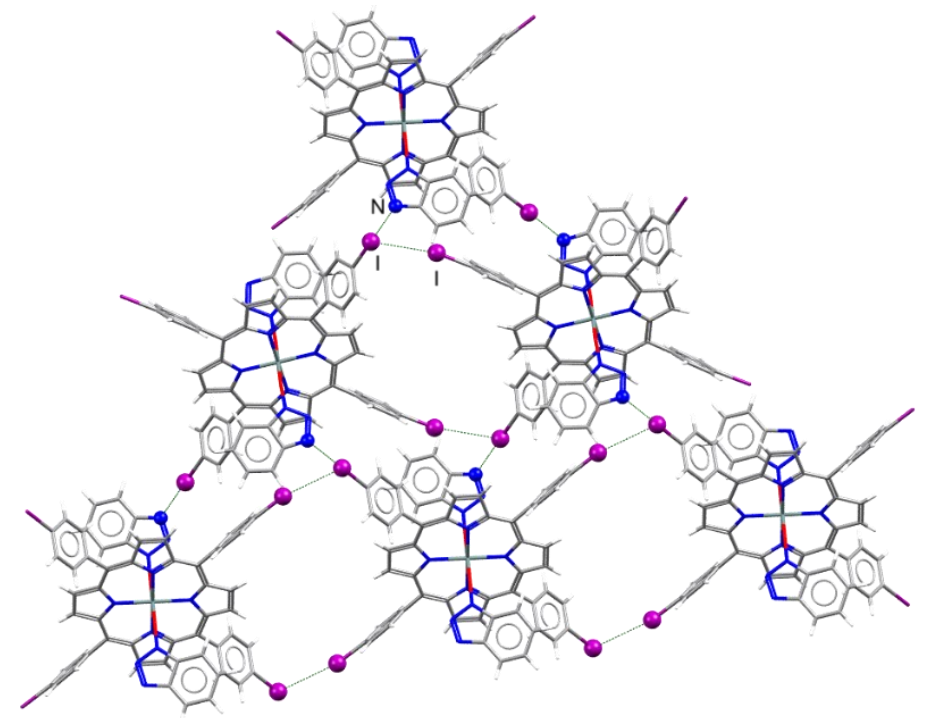

Figure S54. View of the crystal structure with the CSD code HIRWAI, with solvent molecules omitted for clarity. 


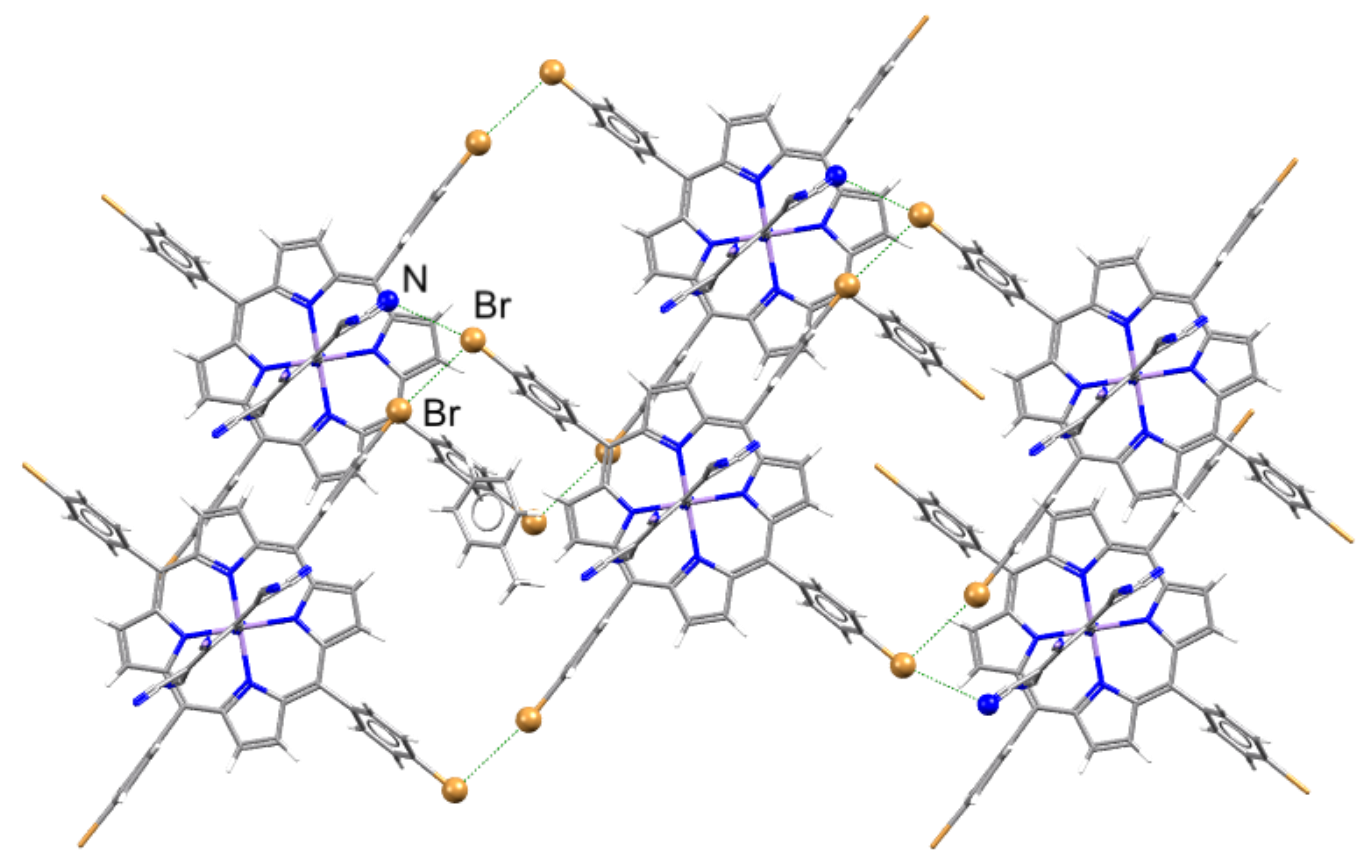

Figure S55. View of the crystal structure with the CSD code QIPPIO.

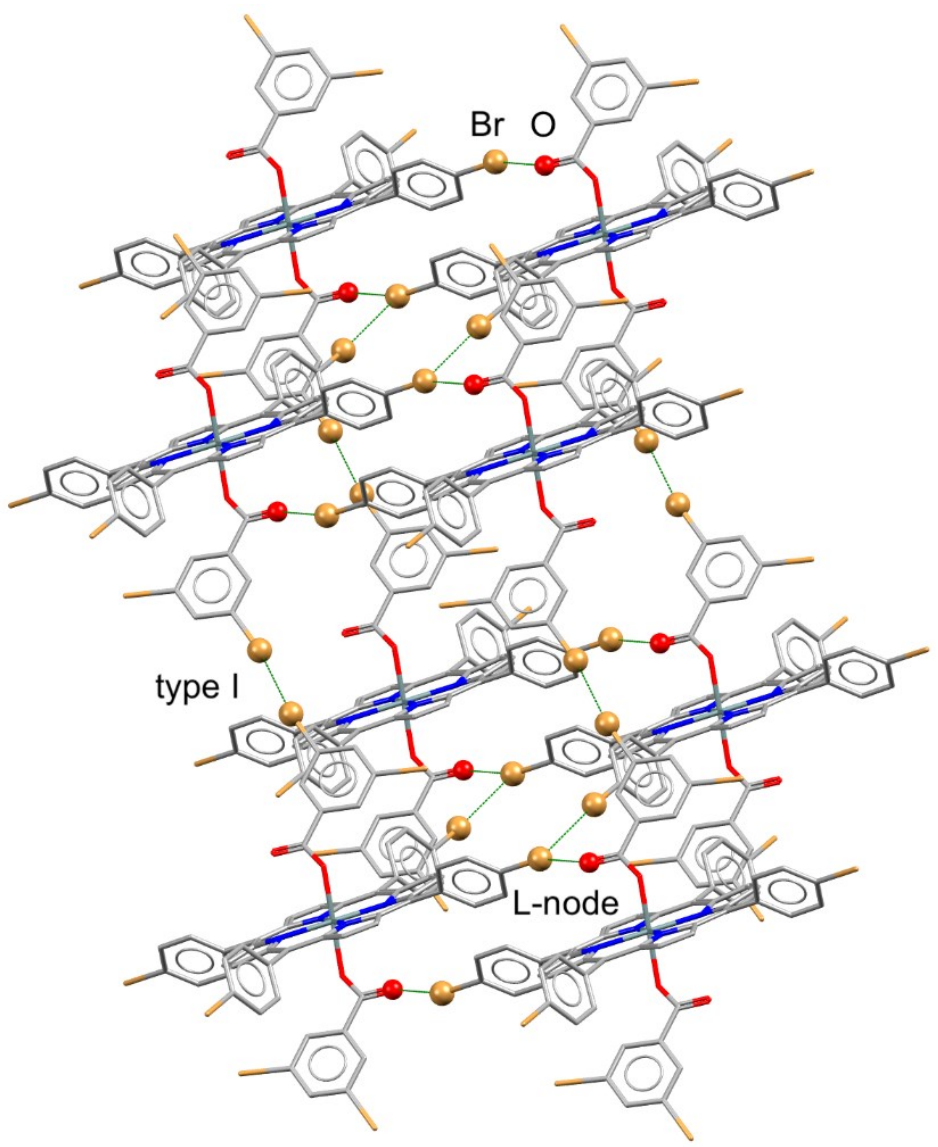

Figure S56. View of the crystal structure with the CSD code XIZLIE 


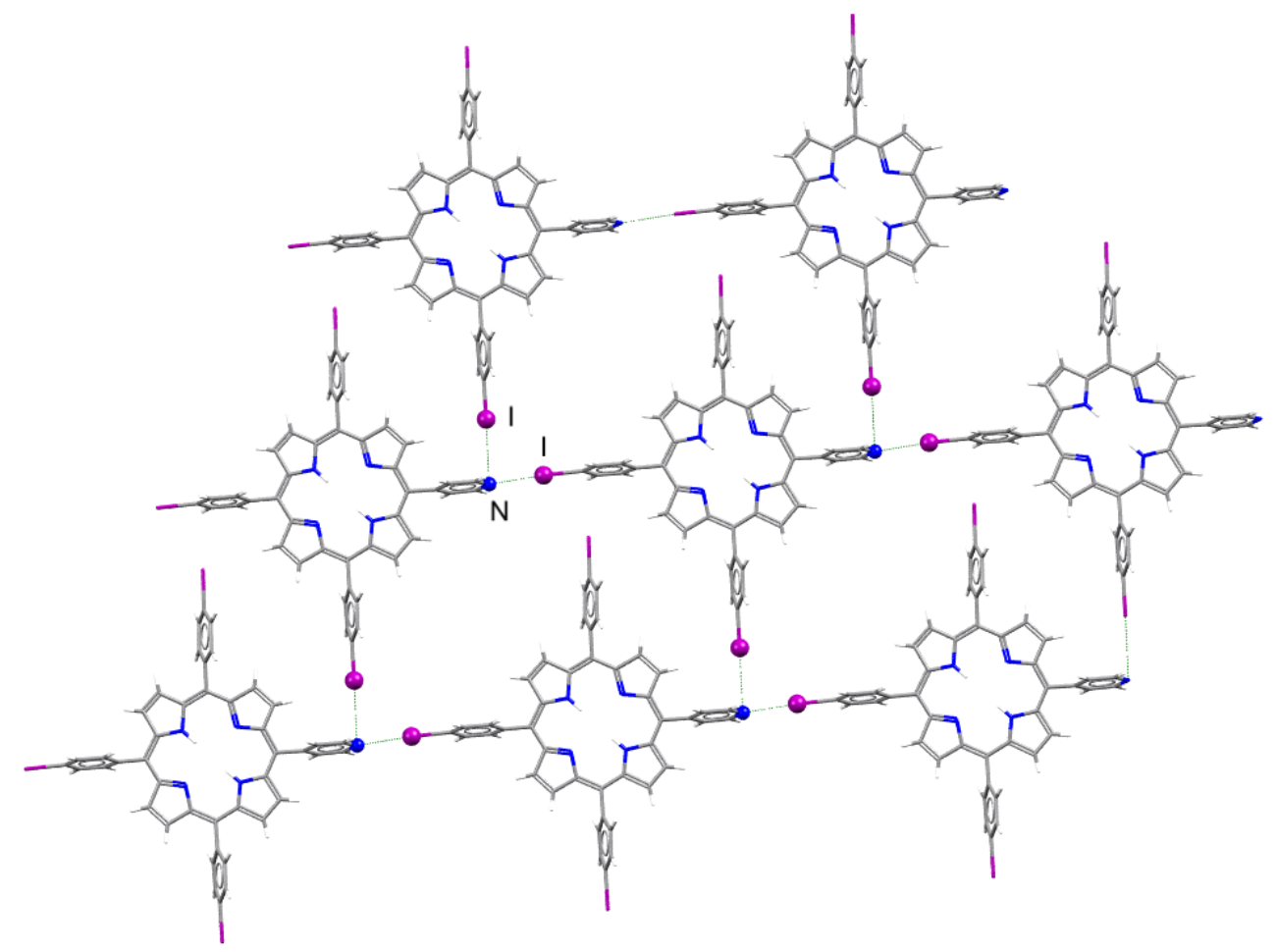

Figure S57. View of the crystal structure with the CSD code SODBET, with solvent molecules omitted for clarity.

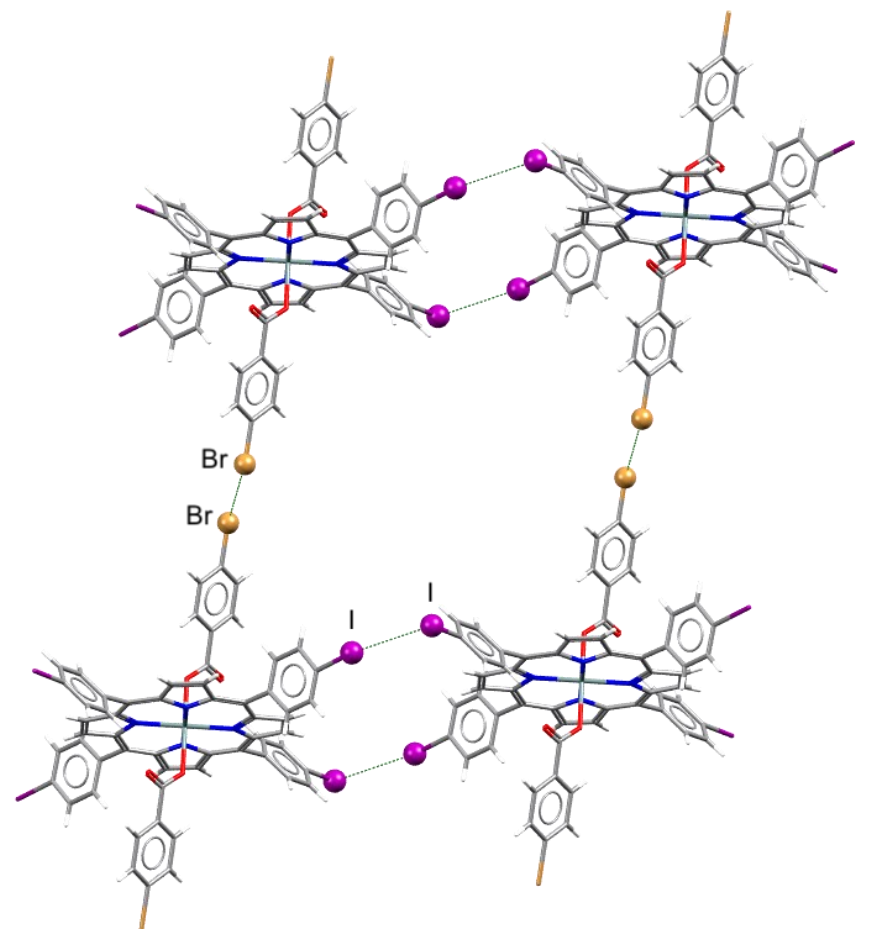

Figure S58. View of the crystal structure with the CSD code KIWTUI, with solvent molecules omitted for clarity. 


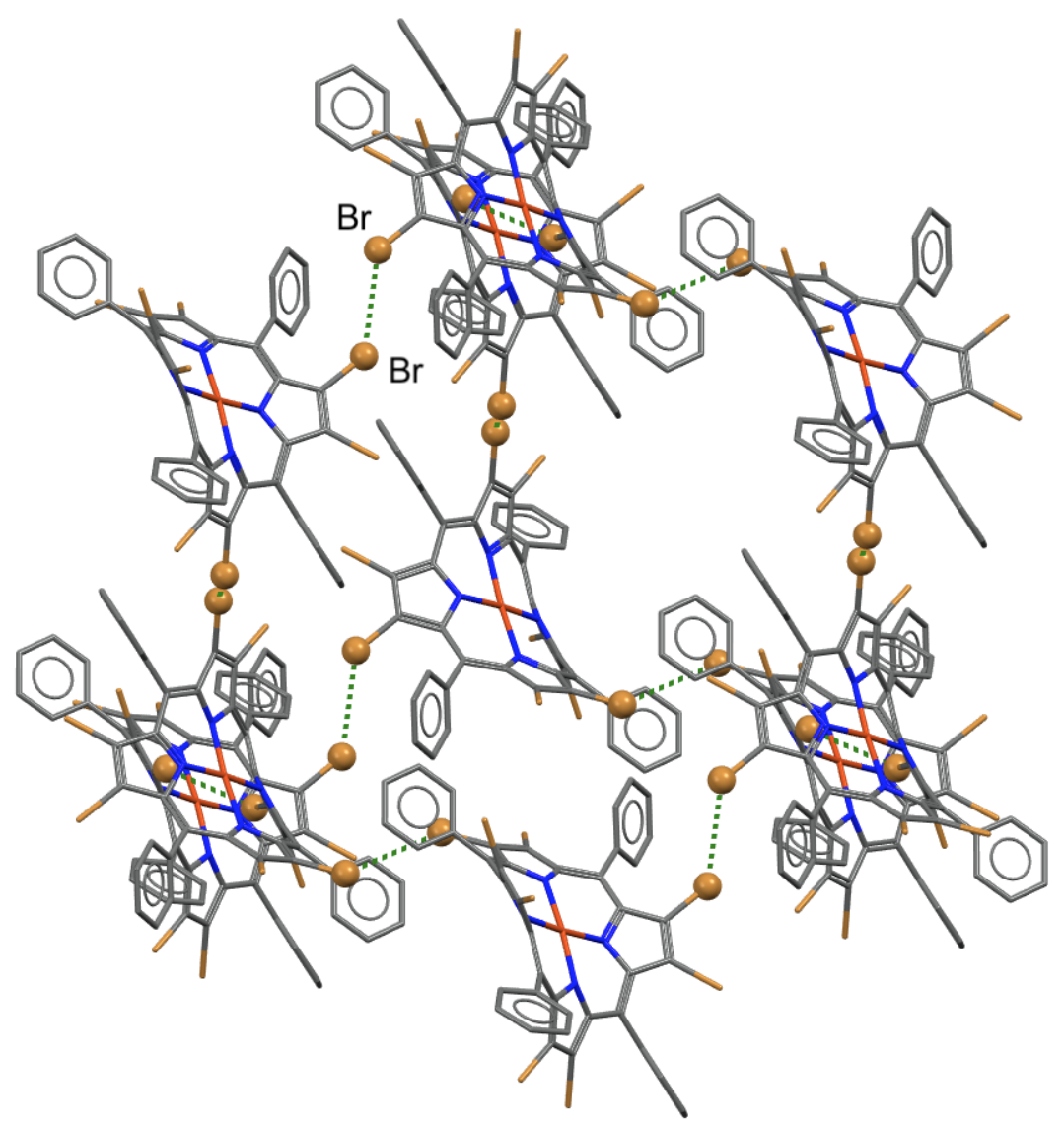

Figure S59. View of the crystal structure with the CSD code SIRLUD, with solvent molecules omitted for clarity.

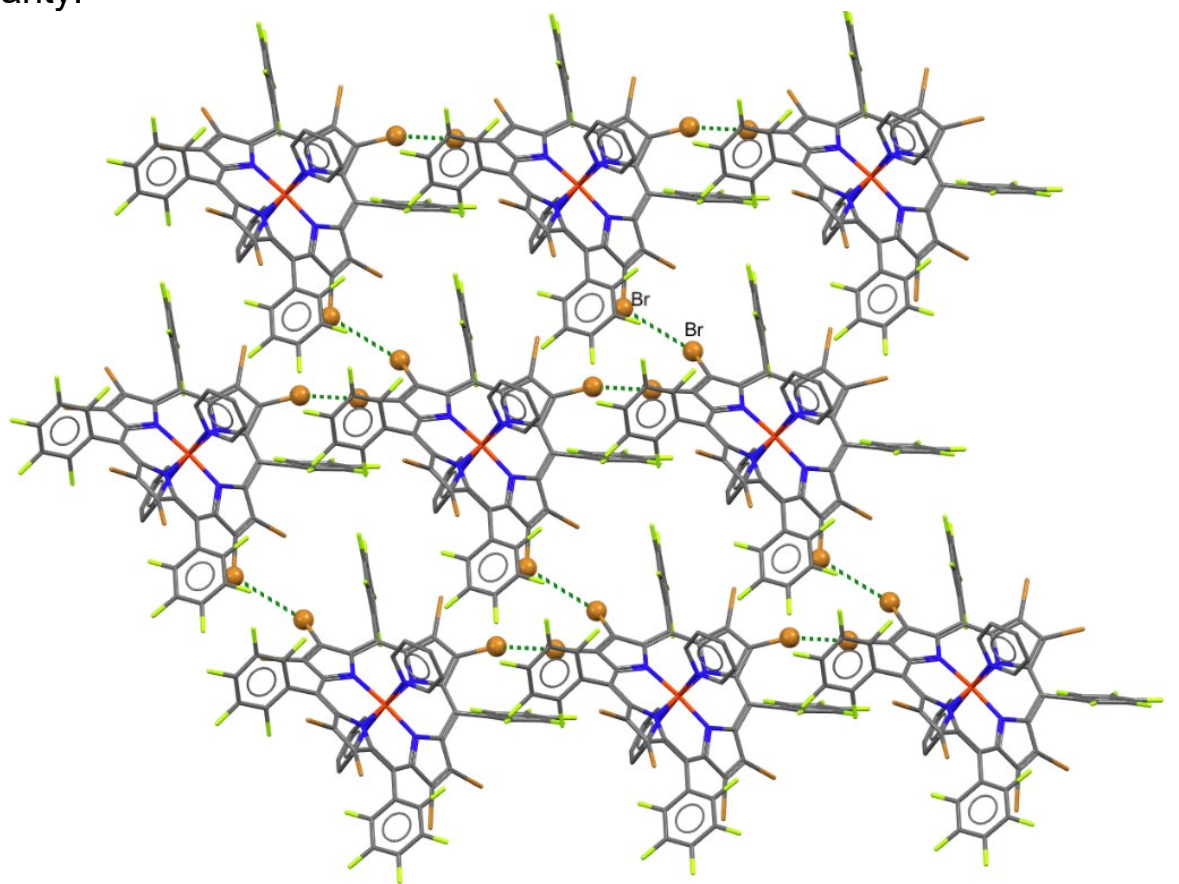

Figure S60. View of the crystal structure with the CSD code ZEQRAO, with solvent molecules omitted for clarity. 


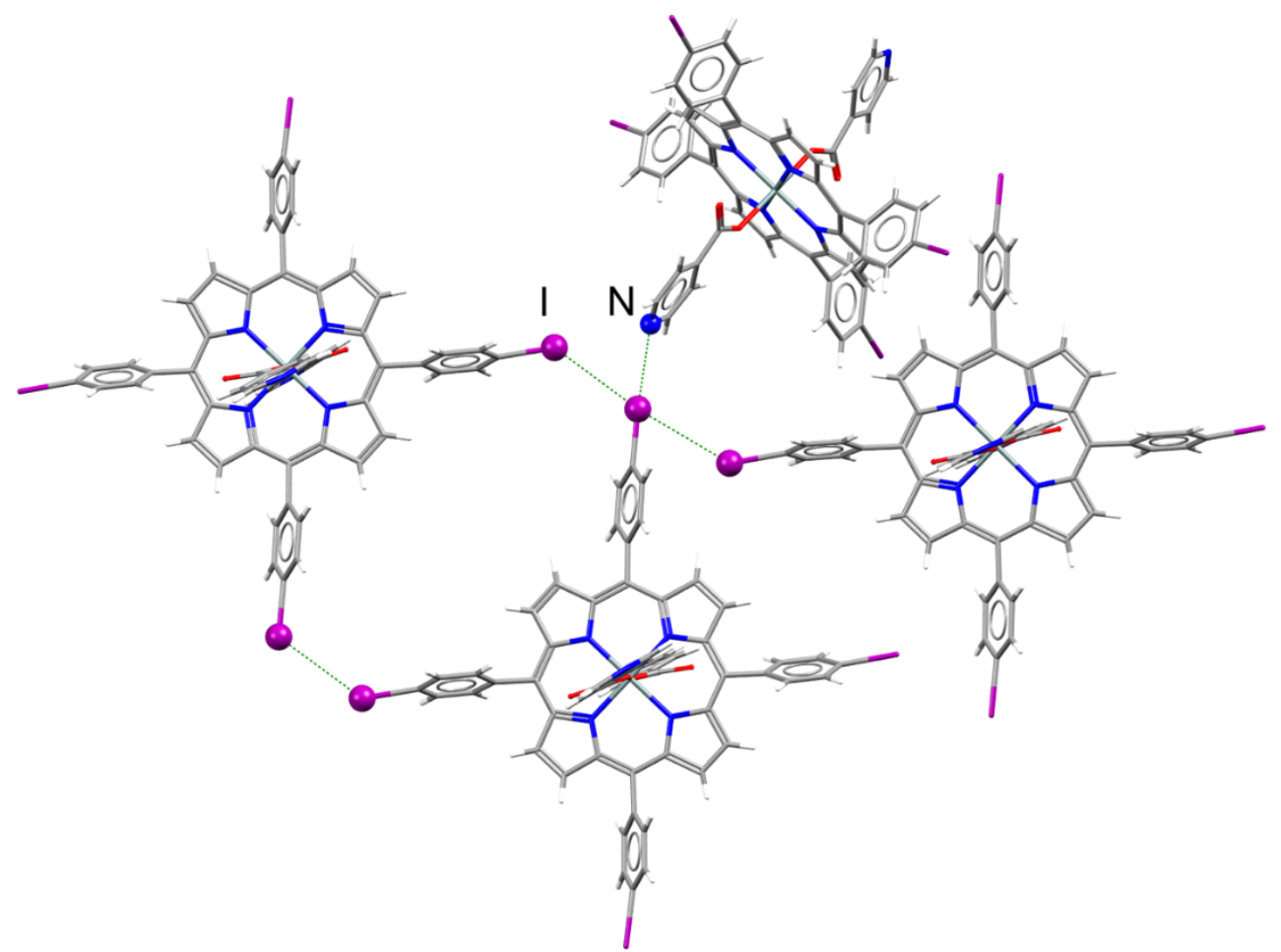

Figure S61. View of the crystal structure with the CSD code HIRVOV.

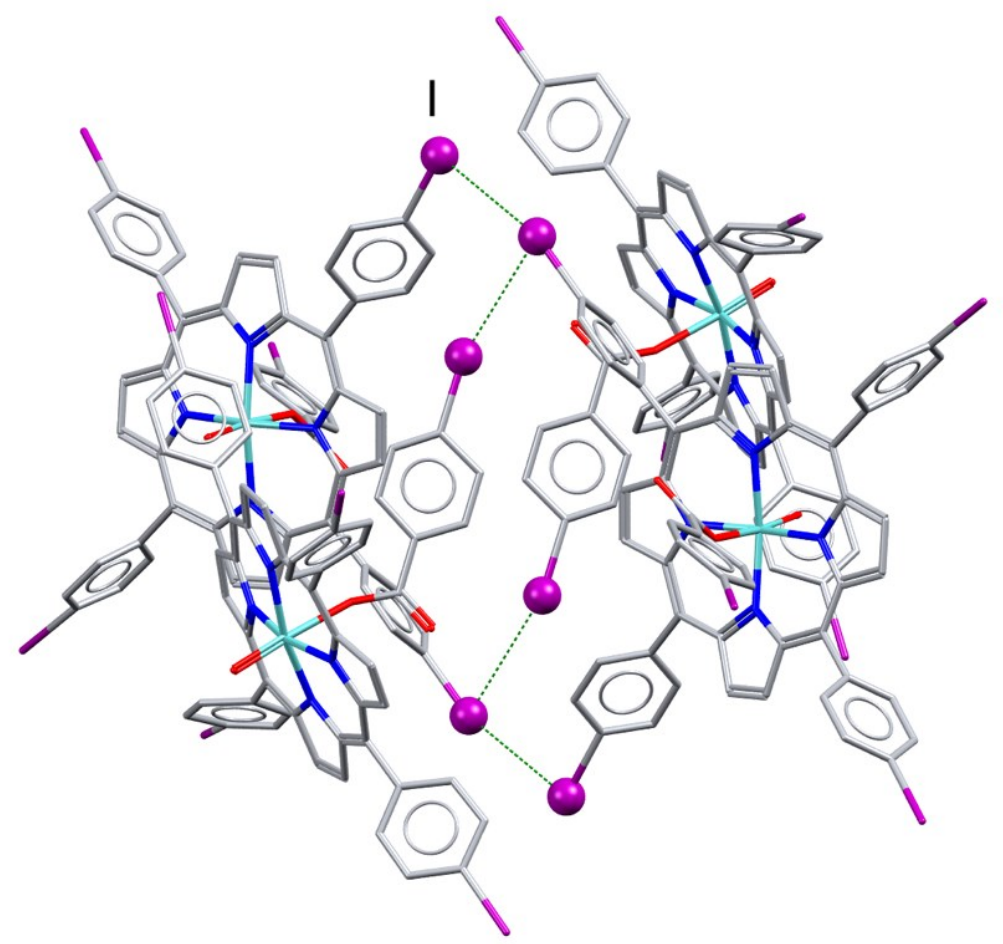

Figure S62. View of the crystal structure with the CSD code VONGOW, with disordered moieties omitted for clarity. 


\section{Hirshfeld Plots}

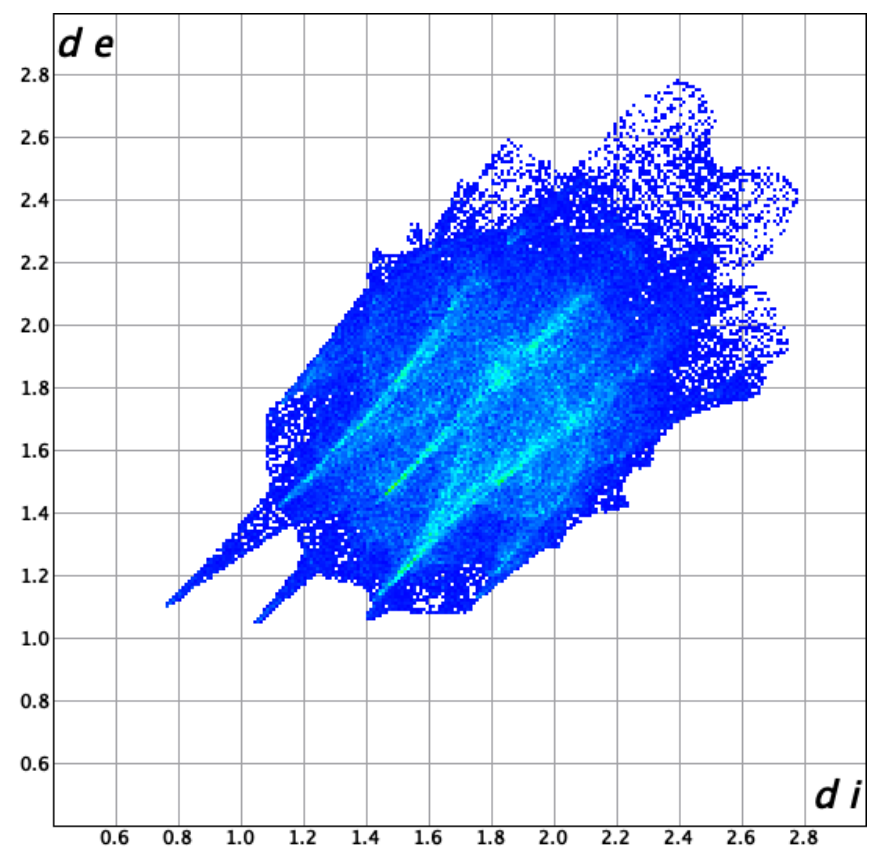

Figure S63. Fingerprint plot for the crystal structure with CSD code AGUYUW

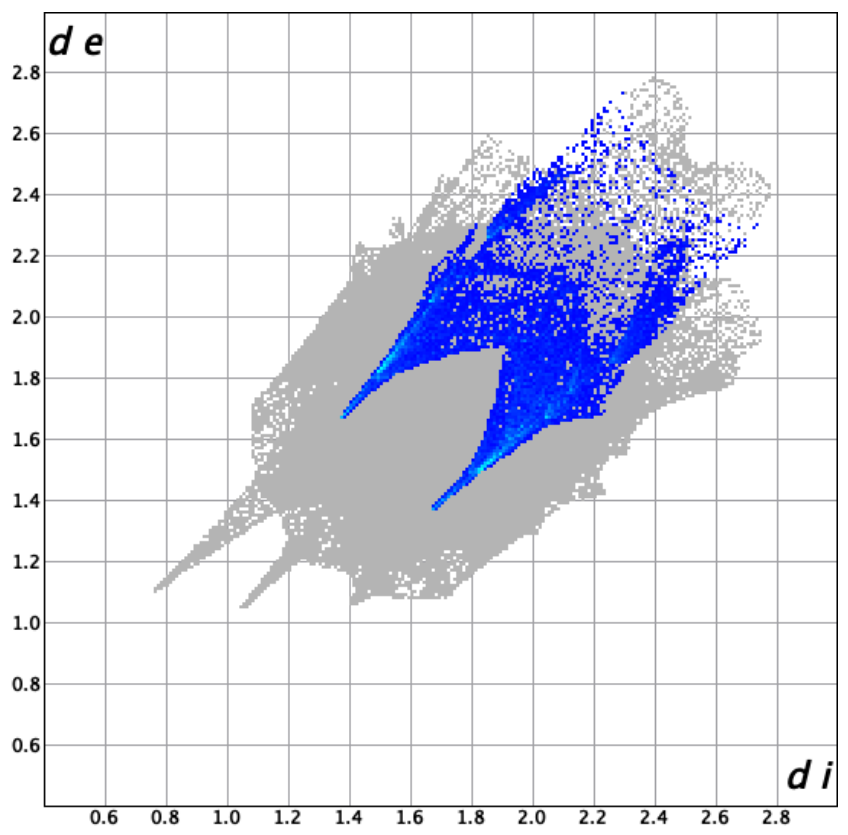

Figure S64. Fingerprint plot for the crystal structure with CSD code AGUYUW, highlighting $\mathrm{Cl} \cdots \mathrm{O}$ interactions 


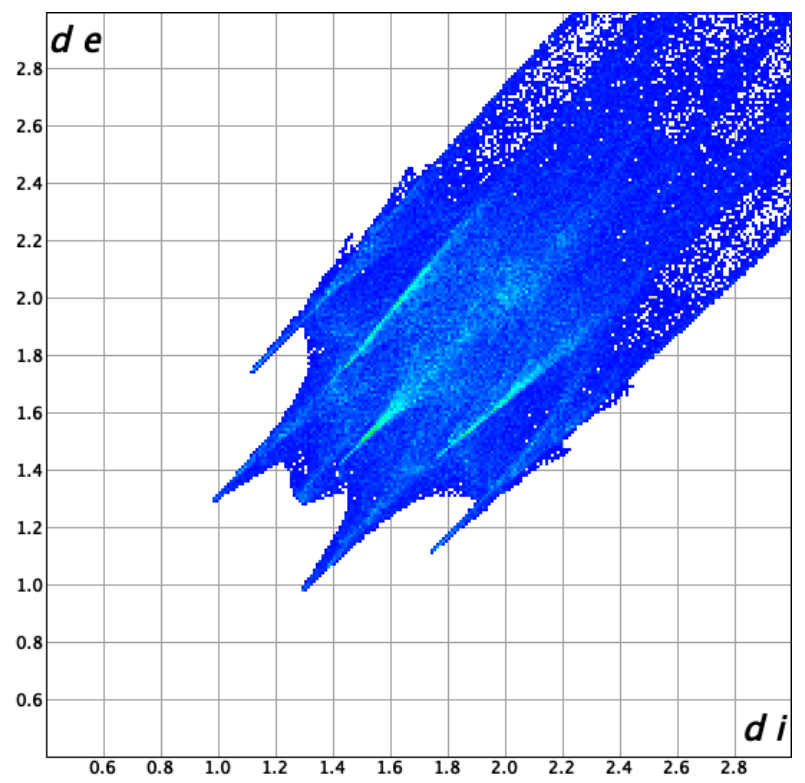

Figure S65. Fingerprint plot for the crystal structure with CSD code XAHMUO.

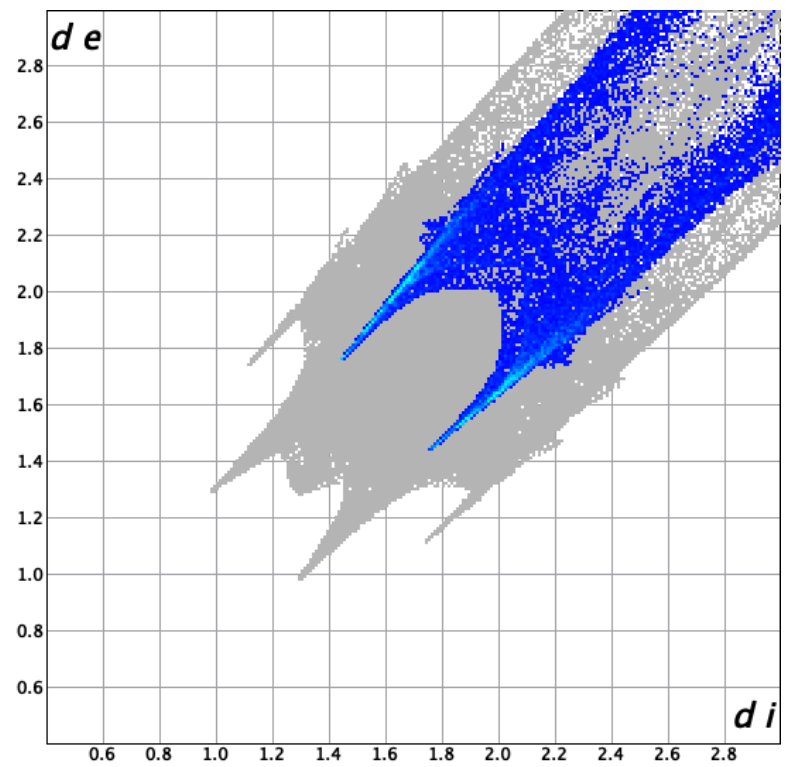

Figure S66. Fingerprint plot for the crystal structure with CSD code XAHMUO, highlighting $\mathrm{Cl} \cdots \mathrm{O}$ interactions. 


\section{Illustrations of the CSD search protocols}

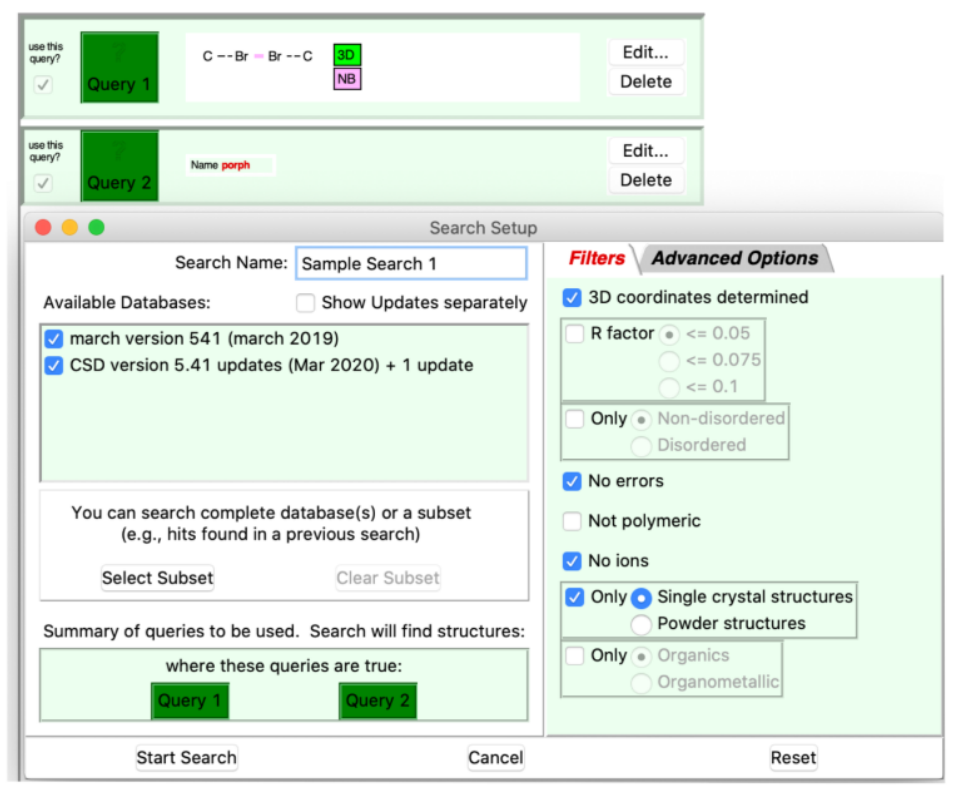

Figure S67. An example of CSD search for halogen $\cdots$ halogen interactions in porphyrin structures. The search is limited to structures with no errors, ions and based on single crystal structures only.

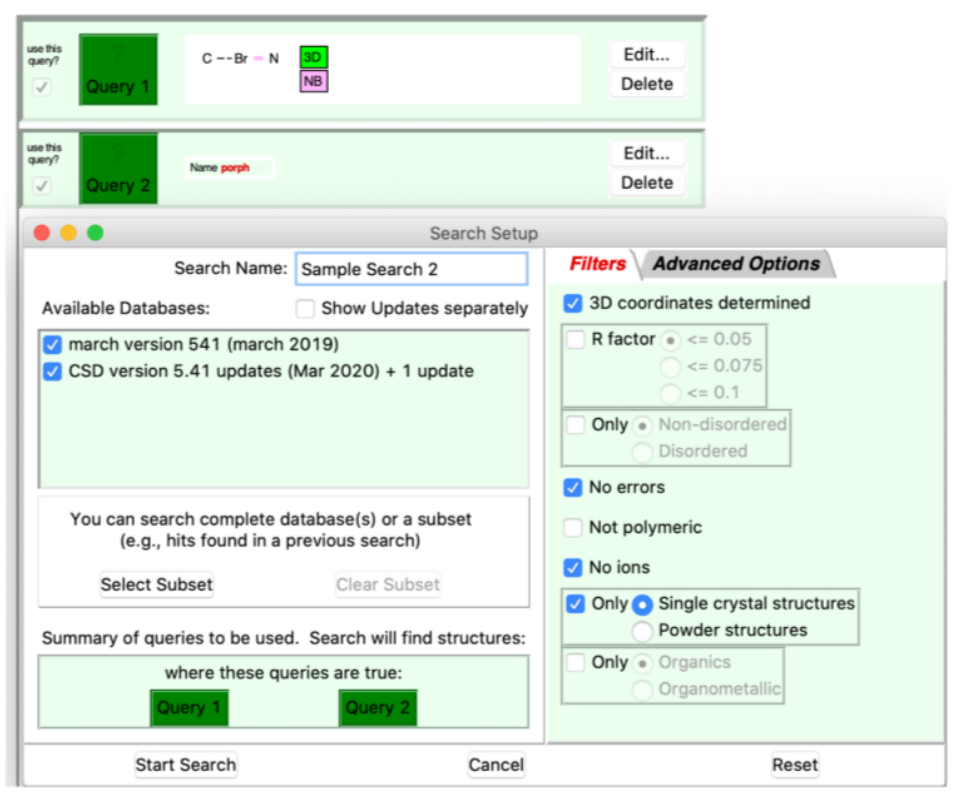

Figure S68. An example of CSD search for halogen $\cdots$ bonding interactions in porphyrin structures. The search is limited to structures with no errors, ions and based on single crystal structures only. 